\title{
Abstracts from Cardiology 2009, 12th Annual Update on Pediatric and Congenital Cardiovascular Disease: Strategies to Improve Care Through a Multidisciplinary Approach, Atlantis Resort, Nassau, Paradise Island, The Bahamas, February 4-8, 2009
}

Extracorporeal Membrane Oxygenation Cardiopulmonary Resuscitation: Nurses Create a Protocol to Save Lives Patrice Wojtyla RN, BSN, NCIII, Alison Boone RN, BSN, CCRN, NCIV Hope Children's Hospital at Advocates Christ Hospital

Background: Our 9 bed Pediatric Surgical Heart Unit (PSHU) has been at the fore-front of providing care for surgical palliation and correction of congenital and acquired heart defects for over 10 years. Due to the high quantity and complexity of the patients treated, our team realized a process to put patients on Extracorporeal Membrane Oxygenation (ECMO) who were unresponsive to traditional CPR was needed. ECPR was developed to cannulate patients rapidly in order to reduce patient complications. As senior staff nurses, our mission was to play a major role in policy development for our unit.

Methods: Literature reviews and research on hospital's ECPR protocols and outcomes was collected. A multidisciplinary team of physicians, perfusionists, ECMO specialists, and nurses formed to discuss a protocol in which patients would be cannulated within 30 minutes. Initially, nursing roles were defined: call, equipment, scrub, circulating, and CPR, all assigned by the charge nurse at ECPR initiation. A specialized pack of gowns and drapes was created with OR staff and put in a mobile cart along with circuit medications, cannulas and connectors. To minimize time, patient rooms were stocked for cannulation, with scrub soaps, sterile gowns, gloves, suction tubing, long-line tubing for arterial lines and emergency numbers. ECMO-specialists were trained to prime the ECMO circuit that sits outside the unit. Badges were prepared defining each role and the steps of the ECPR protocol for staff members to reduce confusion and reinforce the steps. Finally, each staff member had to attend ECPR training and be individually signed off by the ECPR protocol educators. Monthly inservices are held to maintain competencies and each team member is required to attend three a year.

Results: We were able to research, create and initiate the ECPR protocol within 7 months of the initial decision to do so. The multidisciplinary team worked together to be the first pediatric critical care unit in the Chicago area to have such a protocol developed and produce by nurses. Our survival for ECPR was $82 \%$ in 2007, and we achieved a record cannulation time for ECPR of 16 minutes in 2008 with the support of this protocol.

Conclusion: This protocol brought many things to PSHU: a SIMBaby, purchased from the financial support of parents, one whose baby was an ECPR patient, allows us to produce real-time mock ECPR codes; the first Clinical Nurse IV in PSHU obtained her certification based on this protocol; monthly mock codes focusing on our specialized care are held; and both 30 minute cannulations and being involved in protocol development were achieved.

Risk Factors Associated with Obesity after Congenital Heart Surgery

Sara Pasquali MD, Meryl Cohen MD

The Children's Hospital of Philadelphia

Background: Obesity is common in children with congenital heart disease. Little is known regarding traditional and unique risk factors in this population. We evaluated prevalence, risk factors, and co-morbidities associated with obesity in patients (pts) at risk for early atherosclerosis due to past surgery involving coronary reimplantation.

Methods: Ross and arterial switch operation (ASO) pts (6-19yrs) followed at our institution were included; pts with genetic syndromes were excluded. Data was collected from the last clinic visit and pts were sent activity and diet questionnaires. The proportion of obese $(\mathrm{OB}, \mathrm{BMI} \geqslant 95 \%)$ and overweight (OW, BMI 85-94\%) pts was compared to national data with the test of binomial proportions. Other variables were compared in $\mathrm{OB} / \mathrm{OW}$ vs. normal weight $(\mathrm{NW})$ pts using Wilcoxon rank-sum and Chi-square tests.

Results: Of 127 pts, 83\% consented (72 ASO, 34 Ross). Median age was 14.2 yrs (range $6.0-19.9 \mathrm{yrs}$ ); $69 \%$ were male. Similar to national data $(\mathrm{p}=\mathrm{NS}), 14 \%$ were $\mathrm{OB}$ and $17 \%$ were OW. Compared to NW, $\mathrm{OB} / \mathrm{OW}$ pts had higher median systolic blood pressure $[124 \mathrm{mmHg}$ (93-146) vs. $111 \mathrm{mmHg}(90-141), \mathrm{p}=0.02]$, and higher median left ventricular mass index $[48.8 \mathrm{~g} / \mathrm{m} 2.7(24.4-120.6)$ vs. $37.7 \mathrm{~g} / \mathrm{m} 2.7$ $(16.5-85.1), \mathrm{p}=0.01]$. Of the $62 \%(21 \mathrm{OB} / \mathrm{OW}, 45 \mathrm{NW})$ who completed questionnaires there was no difference in $\mathrm{OB} / \mathrm{OW}$ vs. NW pts in activity restriction (physician documented $27 \%$ vs. $27 \%$; parent reported $38 \%$ vs. $42 \%, \mathrm{p}=\mathrm{NS}$ ), or median physical activity [ $9.8 \mathrm{hrs} /$ wk (1.7-41.2) vs. $11.5 \mathrm{hrs} / \mathrm{wk}(0.8-52.3), \mathrm{p}=\mathrm{NS}]$. There was also no difference in history of feeding tube, formula vs. breast feeding, caloric fortification during infancy, or in current caloric intake. OB/OW pts were more likely to have an $\mathrm{OB}$ parent $(70 \%$ vs. $37 \%, \mathrm{p}=0.01)$. Conclusions: OB/OW is common after ASO and Ross and may be related to genetic predisposition, rather than activity restriction or 
early feeding practices. OB/OW pts had higher blood pressure and left ventricular mass, which pose additional long term cardiovascular risk in this unique patient population who underwent coronary artery re-implantation in childhood.

\section{N-Acetylcysteine Attenuates Renal Dysfunction in Neonates Undergoing Arterial Switch Operation \\ Ranjit Aiyagari MD, Sarah K. Gelehrter MD, John R. Charpie MD, PhD University of Michigan}

Background: Cardiopulmonary bypass results in oxidative injury to multiple organs, including the heart and kidney. $\mathrm{N}$-acetylcysteine (NAC) attenuates oxidant stress and preserves myocardial function following ischemia-reperfusion injury in neonatal rabbits. We tested the hypothesis that intravenous NAC attenuates organ dysfunction in human neonates undergoing arterial switch operation (ASO) for d-transposition of the great arteries (d-TGA). Methods: Twenty-one neonates undergoing ASO for anatomic correction of d-TGA and variants were randomized to receive either placebo $(\mathrm{n}=10)$ or a loading dose of $100 \mathrm{mg} / \mathrm{kg}$ NAC followed by a maintenance infusion at $10 \mathrm{mg} / \mathrm{kg} / \mathrm{hr}$ for 24 hours $(\mathrm{n}=11)$. Serial data were collected including thermodilution cardiac outputs, arterial and venous pressures, fluid intake, urine output, chest tube output, inotropic support, serum lactate, time to extubation, time to ICU discharge, and time to hospital discharge.

Results: Baseline demographics were identical between the two groups. Hospital and 30-day survival was $100 \%$, and there were no serious adverse events attributable to NAC administration. Both groups received equivalent inotropic support and diuretic therapy. Compared with placebo subjects, NAC group subjects had a higher total urine output at 24 hours $(169 \mathrm{~mL}$ vs. $89 \mathrm{~mL}$, $\mathrm{p}<0.01)$ and a shorter time to first negative fluid balance $(28 \mathrm{hrs}$ vs. $61 \mathrm{hrs}, \mathrm{p}=0.10$ ) with no difference in total fluid intake or chest tube output between groups. Furthermore, serum creatinine increased by a mean of $0.3 \mathrm{mg} / \mathrm{dL}$ in placebo subjects from preoperative to 24 hours postoperative $(p=0.002)$ but was unchanged in the NAC group. There were trends toward increased cardiac index and lower time-averaged serum lactate in the NAC group. ICU length of stay was shorter in the NAC group by an average of 5 days $(\mathrm{p}=0.02)$.

Conclusion: Perioperative treatment with NAC results in improved urine output, shorter time to achieve a negative fluid balance, and attenuation of the rise in creatinine that normally accompanies cardiopulmonary bypass. The beneficial effects of NAC translate to shorter ICU stays for infants undergoing complex cardiac operations.

Neonatal Complete Repair of Congenital Heart Disease Requiring a Right Ventricle to Pulmonary Artery Conduit Alexander Plato MD, Anthony Rossi MD, Robert Hannan MD, Juan Bolivar MD, Jane Salvaggio ARNP, Redmond Burke MD

Miami Children's Hospital

Background: The physiologic repair of complex congenital heart problems results in early restoration of normal cardiovascular physiology. Repair in some patients has been postponed if the need for continuity between the right ventricle and pulmonary arteries required placement of a conduit (RV-PA conduit). We report our experience of attempted neonatal physiologic repair in patients requiring RV-PA conduits.

Methods: We reviewed our surgical database for all neonates who received RV-PA conduits from June 1995-September 2008. Their perioperative and early postoperative outcomes were evaluated. We excluded patients undergoing single ventricle palliations.

Results: There were 44 pts undergoing RV-PA conduit repairs at an average age of 10.5 days (range 2-29 days) and weight of $3.1 \mathrm{~kg}$ $(2-4.7 \mathrm{~kg})$. There were 22 pts with a diagnosis of truncus arteriosus and 19 with VSD pulmonary atresia. Conduits were constructed from valved homografts in 39 pts (pulmonary15, aortic 24) and bovine heterografts in 2 pts (not described in 3 pts). Median size of homografts was $10 \mathrm{~mm}(7-19 \mathrm{~mm})$. Cardiopulmonary bypass times averaged 176.5 mins $(86-331)$ and aortic cross clamp time averaged 96.8 mins (19-188 mins). Deep hypothermic circulatory arrest was required in only 10 pts (21\%). Delayed sternal closure (DSC) in the CICU was used in 23 pts. DSC was performed at an average of 2.5 days (1-5 days). Conduit size did not determine use of DSC (DSC mean conduit size: $10.1 \mathrm{~mm}$, OR sternal closure $10.3 \mathrm{~mm}$ ). There were 2 hospital deaths (hospital survival 95\%) and no late deaths. Pts were ventilated for a median of 6 days postoperatively (2-50 days). Median postoperative stay was 16.5 days (9-144 days) and hospital stay 26 days (3-140 days). Postoperative cardiac complications included JET, SVT, atrial flutter (1 pt each) and ECMO in 3 pts. One pt requiring postoperative ECMO was placed on ECMO preoperatively. There was no heart block.

Conclusion: Early physiologic repair of congenital heart disease requiring an RV-PA conduit is possible in most patients and associated with low early mortality and morbidity. Deep hypothermic circulatory arrest can be avoided in most pts. The long-term fate of the neonatal RV-PA conduit needs to be determined.

\section{Methods of Increasing Comprehensive and Efficient Patient Care: The Enhancement of a Pediatric Pulmonary Hyper- tension Program \\ Michelle Bagby RN, MSN, CNP, Jean Giver RN, BSN, Russel Hirsch MD Cincinnati Children's Hospital Medical Center}

Background: Inconsistencies in caring for patients through insufficient and disjointed medical care, delays in care plan changes, decision making and duplication of medical work can lead to poor quality patient care and negative outcomes. Given the close monitoring pulmonary hypertension patients require it remains critical for information to be available to the appropriate personnel in an accurate, timely and reliable fashion. As a result of inconsistencies in care and literature review, we have begun implementation of a pediatric health care delivery service where a health care team and sophisticated mechanisms coordinate care. The goal is to efficiently coordinate care and streamline the monitoring process to increase the quality of patient care and the provider's work life and reduce medical errors.

Methods: Our program provides comprehensive, high quality patient care through interdisciplinary team collaboration, including the incorporation of a Nurse Practitioner (NP), use of information technology and professional networking. Cooperation among clinicians is a priority and thus helps to close the knowledge gap of patient care issues and promotes consistency in care and case management. The NPs on the team serve not only as a liaison but as independent providers. Daily rounds encompass the exchange of ideas, including the progress of the patient, plan of care and provide education while including the patient and family. There is also consistency in reviewing serial tests and exams to closely monitor the patients. Utilizing electronic databases provides further comprehensive patient care and increases interdisciplinary collaboration. Results: This model of care is customized to the patient and family. It evolves as needs are anticipated through the development of 
relationships and continuity in care. Quality patient centered care, optimal patient outcomes and patient and family satisfaction are results of the implementation of this program. By utilizing a NP within the program family access is increased because the NP can triage and intervene providing a rapid response and reducing wait time. The use of information technology such as, electronic prescribing and laboratory monitoring, has allowed mainstreaming of medical information, increase in efficiency and reduction of cost and errors while promoting standardization of care. Networking has expanded our patient care treatment options and allows opportunities of being on the forefront of research and new recommendations. This more efficient approach has mainstreamed treatment and increased family access and quality of care. Conclusion: This model facilitates comprehensive and collaborative pediatric medical care, maximizes total patient care, and ensures high quality health care. It assists in timely treatment, informed decision making, efficient resource utilization, reduction of medical errors and increased staff satisfaction.

The Impact of Modified Single Patch Technique on the Left Ventricular Outflow Tract after Repair of Complete Atrioventricular Septal Defect: A Comparative Anatomical Study Anastasios Polimenakos MD, S Bharati, S Shott, CF elZein, M Barth, MN Ilbawi

Heart Institute for Children at Hope Children's Hospital, Rush University Medical Center

Background: Modified single patch technique (MSPT) has been used as an alternative to double patch technique (DPT) in complete atrioventricular septal defect (AVSD) repair. When MSPT is used, left ventricular outflow obstruction (LVOT) is of concern from displacing the atrioventricular valve (AVV) leaflet onto the crest of an already attenuated interventricular septum (IVS). An anatomical comparison between MSPT and DPT was conducted in unoperated specimens to assess their impact on LVOT dimensions.

Methods: Sixteen specimens were examined. First, each had MSPT. The repair was, then, taken down and DPT was performed on all specimens. Each specimen served as its own control. Measurements of LVOT at three levels were performed: 1 at the level of the free edge of AVV anterior leaflet, 3 immediately in the subaortic valve area, 2 at the mid-distance. A and B indicate DPT and MSPT respectively. The depth of the ventricular component of the AVSD (dVSD) from AVV annulus was measured and expressed as a ratio to the total length of IVS (D). Results: $1 \mathrm{~A}$ was $20.67+/-7.05 \mathrm{~mm}$ and $1 \mathrm{~B} 12.33+/-4.96 \mathrm{~mm}$ $(\mathrm{p}<0.001)$. $2 \mathrm{~A}$ was $12.55+/-3.36 \mathrm{~mm}$ and $2 \mathrm{~B} 8.72+/$ $-1.71 \mathrm{~mm} \quad(\mathrm{p}<0.001)$. $3 \mathrm{~A}$ was $8.99+/-2.29 \mathrm{~mm}$ and $3 \mathrm{~B}$ $7.65+/-1.81 \mathrm{~mm}(\mathrm{p}<0.001)$. There was direct correlation between reduction of LVOT at level 1 and dVSD (expressed by $\mathrm{D}$ ratio) when the MSPT was used ( $\mathrm{p} 0.025$, Pearson's $\mathrm{r}$ 0.557). Conclusion: A significant decrease in LVOT is associated with MSPT, especially at level 1, when dVSD is taken into account. Preoperative assessment of the LVOT dimensions, described here, may have implications in surgical strategy. Correlation with clinical findings is warranted.

Depth of Ventricular Septal Defect and Impact on Reoperation for Left Ventricular Outflow Tract Obstruction after Repair of Complete Atrioventricular Septal Defect: Does Double Patch Technique Decrease the Incidence of Left Ventricular Outflow Tract Obstruction? An Anatomical and Clinical Correlation Anastasios Polimenakos MD, S Sathanandam, D Roberson, V Cui, $S$ Bharati, CF elZein, M Barth, MN Ilbawi
Heart Institute for Children at Hope Children's Hospital, Rush University Medical Center

Background: In complete atrioventricular septal defect (CAVSD) left ventricular outflow (LVOT) obstruction is of concern. Modified single patch technique (MSP) has been used as an alternative to double patch technique (DP). Clinical analysis of CAVSD repairs was conducted. Anatomical comparison between MSP and DP in unoperated specimens was performed and the impact of the depth of ventricular septal defect on LVOT assessed. Methods: From September 2002 to August 2008, 77 infants underwent CAVSD repair. Thirteen had MSP and 64 DP. Seven of 13 had trisomy 21 vs 46 of 64 (p ns). Mean age was $4.6+/-1.1$ months (MSP) vs $4.9+/-1.3$ months (DP) (p ns). LVOT peak gradient (PG) and depth of the ventricular component of the AVSD (dVSD) from AV valve annulus were measured by echocardiogram and dVSD expressed as a ratio to the length of ventricular septum from the apex (D). Sixteen anatomy specimens were examined. Each had MSP. The repair was, then, taken down followed by DP. Each specimen served as its own control. Measurements of LVOT were taken: 1 at the level of the free edge of $\mathrm{AV}$ valve anterior leaflet, 3 immediately in the subaortic valve area, 2 at the mid-distance. A and B indicate DP and MSP respectively. Finally, dVSD and D ratio were measured.

Results: Rastelli type A were 47 (10 MSP vs 37 DP), 3 type B (1 MSP vs $2 \mathrm{DP}$ ) and 27 type C (2 MSP vs $25 \mathrm{DP})$. Patients with smaller $\mathrm{dVSD}$ (D ratio) preferentially had MSP $(0.21+/-0.07$ in MSP vs $0.32+/-0.07$ in DP, $\mathrm{p}<0.001)$. Mean follow-up was $36.4+/-2.3$ months. Fifteen patients developed LVOT PG greater than $20 \mathrm{mmHg}$ ( 4 of 13 had MSP, 30.8\% vs 11 of 64 had DP, 20.7\% $-\mathrm{p}<0.05)$. When freedom from reoperation for LVOT obstruction (LVOT PG greater than $50 \mathrm{mmHG}$ ) was analyzed 3 of 13 (23\%) with MSP and 6 of $64(9.4 \%)$ with DP $(\mathrm{p}<0.05)$ required surgical intervention. Seven had modified Konno and 2 subaortic resection. In anatomical comparison, $1 \mathrm{~A}$ was $20.67+/-7.05 \mathrm{~mm}$ vs $1 \mathrm{~B}$ $12.33+/-4.96 \mathrm{~mm}(\mathrm{p}<0.001) .2 \mathrm{~A}$ was $12.55+/-3.36 \mathrm{~mm}$ vs $2 \mathrm{~B}$ $8.72+/-1.71 \mathrm{~mm}(\mathrm{p}<0.001)$. $3 \mathrm{~A}$ was $8.99+/-2.29 \mathrm{~mm}$ vs $3 \mathrm{~B}$ $7.65+/-1.81 \mathrm{~mm}(\mathrm{p}<0.001)$. There was direct correlation between reduction of LVOT at level 1 and dVSD (D ratio) when the MSPT was used ( $\mathrm{p}$ 0.025, Pearson's r 0.557).

Conclusion: MSP is associated with higher incidence of LVOT gradient and lower freedom from reoperation for LVOT obstruction. The impact of dVSD (D ratio) on LVOT, especially at level 1 (as shown in anatomical comparison), can be essential in selecting surgical strategy. Preoperative assessment, as described here, is warranted.

Trisomy 21 and Functional Single Ventricle: Feasibility Study in Patients Undergoing Complete Fontan

Anastasios Polimenakos MD, S Sathanandam, CF elZein, M Barth, MN Ilbawi

Heart Institute for Children at Hope Children's Hospital, Rush University Medical Center

Background: There is scarcity of data regarding indicated management of children with trisomy 21 syndrome (T21S) and a functional single ventricle (FSV). Persistent high pulmonary arterial pressure (PAP) and airway obstruction often can complicate the pathway to completion Fontan (CF). A clinical review of our results for patients with T21S with FSV was conducted to identify factors that may contribute to improved outcome.

Methods: From September 1998 and August 2008, we identified 7 (3 males and 4 females) FSV patients with T21S among 146 who 
had undergone BDG and of which 138 had CF. Mean age at BDG was $33.3+/-14.6$ weeks (16 to 59) and at CF $28.5+/-12.6$ months (19 to 47). Mean Body weight at CF $26.8+/-8.8 \mathrm{~kg}(15.7$ to 36.1). All 7 patients had unbalanced complete atrioventricular septal defect. Four had dominant left ventricle and 3 dominant right ventricle. One had subaortic stenosis.

Results: There were no hospital deaths with one mortality prior to CF. No BDG or CF were taken down. Pulmonary artery banding $(\mathrm{PAB})$ was the initial procedure in 6 patients. $\mathrm{PAB}$ was adjusted for PAP $20 \%$ of systemic arterial pressure. One patient had aortopulmonary shunt. All patients progressed to pulsatile BDG and one had Damus-Kay-Stansel with BDG. Median Follow-up was 53 months (18 to 93). Four of six survivors after BDG underwent a subsequent $\mathrm{CF}$ and 2 awaiting $\mathrm{CF}$ with none having significant risk factors to prevent CF. Two of CF were fenestrated. Both had their fenestration closed in average 3 years post-CF. All CF patients treated with aspirin and warfarin postoperatively. One patient after CF had AV valve repair for severe insufficiency with no further sequelae. No arrhythmias, protein-losing enteropathy, AV node block or ventricular dysfunction were noted during the follow-up period.

Conclusion: Trisomy 21 syndrome has been reported as a risk factor in patients with FSV, mainly, due to persistent high PAP. This feasibility study shows that single ventricle pathway repair in patients with T21S can be followed safely with encouraging results. Early tight $\mathrm{PAB}$, and continued pulmonary vascular maturation due to pulsatile BDG may contribute to this outcome. Careful patient selection for CF is warranted.

Implementing the Night Educator Role in a Pediatric Cardiac Intensive Care Unit

Ruth Brediger RN, MSN, CCRN, RRT, Jeanne P. Ahern BSN, RN, CCRN, Patty Hickey PhD, RN, MBA, CNAA

Children's Hospital Boston

Background: Traditionally, educational programs and opportunities for clinical advancement are limited for nurses working the night shift. This creates a predicament because there is a greater proportion of less experienced staff working nights and, in a pediatric cardiac intensive care unit, patient acuity is very high immediately after cardiac surgery at a time when system-wide resources are low.

Aim: To describe the development of the night clinical educator role and its impact of the availability of educational opportunities in a Pediatric Cardiac Intensive Care Unit at Children's Hospital Boston.

Methods: First, 39 pediatric cardiac ICU nurses working night shift were asked to complete a needs assessment. Results were then used to develop an educational plan that included an education committee. The plan was based on Knowles' Adult Learning Theory. Specifically, the night educators' role was created to facilitate learning and growth that empowered the nursing staff to take an active role in their continuing education and professional development. The nursing staff was encouraged to create then present educational topics that they identified as relevant and important to them.

Results: $64 \%$ of night staff nurses responded to the needs assessment. Pacemakers, atrial wire tracings and emergency procedures were identified as the three most pressing educational needs. An education committee was created to address these needs. The number of educational presentations increased from 42/12 months in 2007 to $52 / 9$ months in 2008 . The number of nurses participating in the education of staff grew from 6 nurses in all of 2007 to 27 nurses in only 9 months in 2008; this represents a four fold increase in participation by staff nurses. As well as the three top educational needs, additional educational activities included emergency scenarios, nursing rounds, monthly educational topics, emergency equipment competencies, and a night journal club. The education committee currently has 38 active members; these members educate and introduce new products to nurses on all shifts and weekends.

Conclusion: The night educator role can have a significant impact on facilitating the availability of professional development opportunities for nurses working during the night shift.

\section{Electrical Seizures are Common in Neonates During Stage 1 Palliation for Hypoplastic Left Heart Syndrome without Circulatory Arrest Julia Gunn MBBS, FRACP, Rod Hunt, Shelly Lavery, Stephen McKeever, John Beca, Terrie Inder, Lara Shekerdemian Murdoch Children's Research Institute, Royal Children's Hospital, Starship Children's Hospital}

Background: Neonates undergoing cardiac surgery are at significant risk for early brain injury. The detection of seizures following neonatal cardiac surgery has been associated with impaired neurodevelopmental outcome. The incidence of intra-operative seizures in these infants, however, is currently unknown. We aimed to determine the incidence of peri-operative seizures in neonates during stage 1 palliation for hypoplastic left heart syndrome (HLHS).

Methods: As part of a prospective study of brain injury in infants with congenital heart disease, a group of neonates undergoing stage 1 palliation for HLHS underwent peri-operative two-channel amplitude integrated EEG (aEEG) monitoring. aEEG was undertaken prior to and during induction of anesthetic, throughout surgery and for 72 hours post-operatively on the intensive care unit. Our institutional perfusion strategy for stage 1 palliation included moderately hypothermic cardiopulmonary bypass (CPB) with selective cerebral perfusion of the innominate artery at $120-150 \mathrm{~mL} / \mathrm{min}$, without circulatory arrest. A blinded assessor reviewed the aEEG recordings for seizure activity in each cerebral hemisphere. Suspected seizures were confirmed on the raw EEG trace and classified as single seizure (SS), repetitive seizures not meeting the criteria for status epilepticus (RS) or status epilepticus (SE).

Results: Twenty full-term neonates with HLHS (mean birth weight $3.3 \pm 0.6 \mathrm{~kg}$ ) underwent stage 1 palliation at a median age of 2 days (range 1-7). The mean minimum esophageal temperature during CPB was $24.5 \pm 1.4{ }^{\circ} \mathrm{C}$. Five participants died prior to the second stage of surgical palliation. Nine infants (45\%) had aEEG evidence of peri-operative electrical seizures without any clinical correlate. Seven infants experienced intra-operative seizures. Of these, pre-CPB intra-operative seizures occurred in one (SS). Seizures were present during hypothermic CPB in 5 infants (1 SS, $4 \mathrm{RS}$ ), and occurred during rewarming in 3 (1 SS, 1 RS and $1 \mathrm{SE}$ ). Intra-operative seizures did not recur following re-establishment of normothermia. Intra-operative seizures were predominantly left-sided. Post-operative seizures were present in 5 infants (3 SS and 2 RS). Peri-operative seizures were present in 4 of the 5 infants who died. These occurred during the post-operative period (2 patients), or during both the intra-operative and post-operative periods (2 patients).

Conclusion: This is the first description of intra-operative seizures in neonates undergoing complex cardiac surgery. Seizures are common in these infants, and were shown to predominantly affect the left hemisphere during isolated cerebral perfusion at moderate hypothermia. Further follow up of the survivors is essential to determine the long-term significance of these findings. 
Presurgical Enteral Feeding in Neonates with ProstaglandinDependent Congenital Heart Disease: Preliminary Results of an International Survey on Current Trends and Variations in Practice

Lisa Willis MD, Jon Kaufman, Patti Thureen, Erica Wymore, Eduardo da Cruz

The Children's Hospital of Denver, University of Colorado Denver, School of Medicine

Background: Safety of enteral feeding in term neonates with congenital heart disease (CHD) on intravenous prostaglandin (PGE1) therapy has not been determined. Benefits of early enteral nutrition in term and pre-term infants with critical illness are well known, and it is thought that an improved preoperative nutritional status in the neonate with CHD may lead to improved surgical outcomes, fewer hospital complications, and shorter length of stay. However, the paucity of data regarding optimal preoperative nutrition in term PGE1dependent neonates has led to a wide variety of nutritional practices for this population. Infants with PGE1-dependent congenital heart disease require a patent ductus arteriosus to perfuse either their systemic or pulmonary vascular bed while awaiting surgical repair. The pattern of ductal flow is often considered when determining the safety of enteral feeding in this population. This survey was designed to assess the differences in presurgical enteral feeding practices around the world, and to determine if the neonate's ductal flow pattern plays a role in the provider's decision to enterally feed these patients.

Methods: In March 2008, pediatric practitioners were contacted via email to respond to an internet-based survey using commercially available software (www.surveymonkey.com). Responses were collected regarding the preoperative nutritional practices of neonates with PGE1-dependent cardiac disease. No attempt was made to correlate nutritional management practices with outcomes.

Results: A total of 200 caregivers responded to the survey with $79.5 \%$ United States (US) and $20.5 \%$ international respondents. Respondents reported 109 different US zip codes and represented 22 countries. Physicians represented $71 \%$, NP/PAs 10\%, nurses $11.5 \%$, and other medical personnel $7.5 \%$ of responders. In the US, 115/145 (79\%) medical caregivers reported enterally feeding preoperative PGE1-dependent neonates in comparison to 38/41 (93\%) of international respondents. A total of $76 / 145$ (52\%) US respondents and 27/41 (66\%) international respondents reported that ductal flow pattern does not impact their decision to enterally feed. For those caregivers whom ductal flow pattern affects enteral feeding decisions, 25/145 (17\%) US respondents and 8/41 (20\%) international respondents preferred enterally feeding PGE1dependent neonates with a left-to-right ductal flow pattern.

Conclusion: A majority of the respondents, both US and international, reported early enteral feeding in preoperative PGE1-dependent neonates. More than half the respondents fed independent of ductal flow pattern. For those providers who use ductal flow direction to determine the safety of enteral feeding, a left-to-right ductal flow pattern was preferred. The pathophysiology of left-to-right ductal shunting would suggest though, that this group of infants may be more likely to experience mesenteric ischemia secondary to retrograde diastolic flow in the descending aorta. In addition, there was a minority of respondents who do not enterally feed any neonate receiving intravenous PGE1 therapy.

These partial results of the survey emphasize the variability in the current nutritional management of neonates with PGE1dependent cardiac disease. The impact of these preoperative nutritional practices is as yet unknown. Our survey demonstrates the need for prospective data collection on enteral feeding strategies and their impact on the nutritional status in these infants.
Adherence to Guidelines for Cardiovascular Screening in Current High School Preparticipation Evaluation Forms Christopher Rausch MD, George Phillips $M D$ The Children's Hospital, Denver; The University of Iowa Children's Hospital

Background: Preparticipation screening for cardiovascular abnormalities that may predispose to sudden cardiac death continues to be an important area of discussion and debate among both lay and medical communities. Due to concerns regarding the cost inneffectiveness of other screening methods, the preparticipation examination has been deemed the most practical tool to aid in the reduction of sports related sudden cardiac death. The purpose of this study was to evaluate the content from each state high school athletic association's approved, recommended, or required preparticipation evaluation form when compared with current consensus cardiac screening recommendations.

Methods: The high school athletic association's approved, recommended, or required sports preparticipation form from each of the 50 United States and the District of Columbia were reviewed and the content of the personal and family history components was compared with current recommendations for cardiac screening questions.

Results: When current preparticipation forms were evaluated based upon the criteria used in prior studies, they were found to include these elements $85 \%$ of the time. When compared with current recommendations, however, we found that present preparticipation forms completely address these recommended questions only $17 \%$ of the time.

Conclusion: While there appears to be some improvement in the content of the preparticipation forms in use compared with prior studies, the vast majority are incomplete when compared with current consensus guidelines.

Transport Of Critically Ill Neonates With Cardiac Conditions Jan Hau Lee MBBS, Putbucheary Janil

KK Women's and Children's Hospital, Singapore

Background: A significant number of neonates with cardiac conditions need a specialist team for transfer to a tertiary referral center for further medical and surgical management. Transport care forms an important aspect of intensive care among this group of critically ill neonates.

Methods: Data was collected prospectively on all patients referred to the Children's Hospital Emergency Transport Service (CHETS) at KK Women's and Children's Hospital, Singapore, regarding diagnosis, complications, treatment, physiologic variables, and transport related outcomes. A retrospective review of the data from January 2002 to June 2008 (78 months) identified 50 patients with a primary cardiac disorder. The neonatal subgroup within these 50 patients was then analyzed.

Results: Four-one (82\%) was in the neonatal age group. Thirty-two (78\%) had cyanotic congenital heart conditions (the commonest diagnosis being transposition of great arteries); nine (22\%) had acyanotic heart conditions (the commonest diagnosis being coarctation of aorta). Eight were premature infants (31-36 weeks gestational age). Nineteen $(46 \%)$ neonates were started on a prostaglandin infusion for suspected duct dependent lesions by the referring physician. Thirteen $(68 \%)$ of patients who were on prostaglandin were not mechanically ventilated and there were no respiratory complications. There were no intra-transfer adverse events in this group of patients. There were 4 transfers by fixed wing aircraft (overseas transfers). One case involved a palliative transfer for comfort care at home. 
Conclusion: Our results are consistent with previous studies in that mechanical ventilation may not be necessary for the transport of babies who are started on prostaglandin infusion. CHETS is safe and effective in transporting neonatal cardiac patients even among premature infants.

\section{Perioperative Risk Factors for Acute Renal Failure after} Infant Cardiac Surgery

Jennifer Hirsch MD, MS, Alice Chi BS, John Charpie MD, PbD, James Gurney PhD, Janet Donobue MPH, Karen King BA, Richard Obye MD, David Kershaw MD, Robert Gajarski MD University of Michigan, CS Mott Children's Hospital

Background: Acute renal injury after cardiac surgery is associated with significant morbidity and increased mortality risk. Predictive risk factors have not been well described. The purpose of this study was to identify perioperative factors associated with acute renal failure (ARF) requiring dialysis in a group of infants undergoing congenital heart surgery.

Methods: This IRB approved, case-controlled study was conducted among infants (age 0-12 months) with congenital heart disease. Cases were those surgically treated from $7 / 2002$ to $7 / 2008$ who developed postoperative ARF requiring dialysis $(\mathrm{N}=42)$, and control subjects $(\mathrm{N}=118)$ who were frequency-matched to cases by age and treated during the same time period. Data collected included demographics, renal anatomical abnormalities, cardiac diagnoses, type of surgery with risk-adjusted congenital heart surgery (RACHS) scores, baseline and post-operative creatinine (Cr), vasopressor support [including inotrope score (IS)], cardiopulmonary bypass (CPB) times and hospital mortality.

Results: Median overall age and weight at surgery was $15 \mathrm{~d}$ and $3.8 \mathrm{~kg}$, respectively. Incidence of anatomic renal abnormalities in ARF pts was $30 \%$. Compared with controls, those with ARF requiring dialysis were more likely to have pre-operative $\mathrm{Cr}>1.0$ (odds ratio $[\mathrm{OR}]=22.9 ; \mathrm{p}=0.004$ ) or high $\mathrm{RACHS}$ score (RACHS 5-6 vs. $1-2, \mathrm{OR}=2.8 ; \mathrm{p}=0.05$ ). Intra-operative factors associated with ARF included longer CPB times (134.2 vs. $97.3 \mathrm{~min} ; \mathrm{p}=0.001$ ) and multiple vasopressor agents ( 4 vs. 1 , $\mathrm{OR}=16.5 ; \mathrm{p}<0.001)$. During the initial 24-hrs post-op, infants with ARF were more likely to have an IS $>15$ (OR 5.3; $\mathrm{p}>0.001), \mathrm{Cr}>1.0(\mathrm{OR} 40.7 ; \mathrm{p}<0.001)$ or to die $(62 \%$ vs. $7 \%$, $\mathrm{p}<0.001)$ compared with controls.

Conclusion: Risk factors for ARF requiring dialysis among infants undergoing cardiac surgery include RACHS scores $>4$, use of multiple vasopressor agents following $\mathrm{CPB}$ or a peri-operative $\mathrm{Cr}$ $>1.0$. To mitigate morbidity and mortality, further studies are warranted to evaluate therapies aimed at preventing acute renal injury after cardiac surgery in infants.

\section{Sneak Peaks to PEAK Streams: Performance Excellence Acquired through Knowledge Kirsten Schroeder RN, Julie Vaandrager British Columbia Children's Hospital}

Background: At British Columbia Children's Hospital it is a high priority to focus on paediatric nursing professional development to support a quality practice environment. A paediatric nursing professional development plan was created through a competency based education approach and implemented within a personal accountability framework.

The need for a competency based learning plan was determined in conjunction with the re-structuring of the hospital inpatient units, where specific specialities were combined. This paediatric nursing professional development plan was designed to meet foundational, advanced and complex levels of nursing competencies. Building on foundational paediatric nursing competencies, Sneak Peaks to PEAK Streams were implemented as the next step in a paediatric nurse's career path.

Methods: Upon employment at British Columbia Children's Hospital, a nurse will complete a Paediatric Nursing orientation, which includes education sessions to give a "Sneak Peak" into the sub-specialties. After a personal evaluation of foundational competencies, a nurse can enter into a "PEAK" Stream (Performance Excellence Acquired through Knowledge) to focus on advanced and expert competencies.

Sneak Peaks and PEAK Streams were developed in all sub-specialty areas, including cardiology, neurology, nephrology, oncology, and others. PEAK Streams are divided into three six-week learning opportunities based on the concept of adult learning, where newly acquired knowledge is applied in daily practice and learning is experiential, self directed and competency based.

Results: This presentation will outline how the Cardiology PEAK Stream was recently redeveloped to reflect learning needs identified by nursing leadership and frontline staff. The presentation will explore the expectations of a PEAK Stream including the following details:

$80 \%$ of the learning involves the nurse providing clinical care to primarily cardiology patients.

$20 \%$ of time is dedicated for alternate learning experiences, such as observing ECHOs, Cath Lab, ICU, Cardiovascular Surgery or working through recent research articles.

Optional learning activities are provided based on the daily identified learning goals of the nurse.

Conclusion: The goal of PEAK streams is to provide a professional development path that will help nurses develop foundational competencies in paediatric cardiology specialty nursing practice. It has the potential to greatly improve the competency of frontline nurses as they provide care to the patients of British Columbia Children's Hospital.

Is Bypass or Cross Clamp Time Independently Associated with Acute Renal Failure or Mortality in Pediatric Post-Operative Cardiac Patients?

Phil Spinella MD, Troung D, Stoltz P, Berning $R$, Schlott $H$,

Kellerman K, Carroll CL

Connecticut Children's Medical Center

Background: Acute renal failure (ARF) can significantly increase morbidity and mortality following cardiac surgery in adults. However, few studies have examined ARF in children following cardiac surgery. Our objective was to determine if bypass time (BPT) or cross clamp time (CCT) were independently associated with the development of ARF or mortality in children following surgery for congenital heart disease.

Methods: We conducted a retrospective review of children who underwent cardiac surgery between August 2005 and August 2008. Only children who required both bypass and cross clamping were included. Acute renal failure was defined as serum creatinine $>1.5$ times baseline or treatment with peritoneal or hemodialysis. Other predictors and outcomes analyzed included age, race, Risk Adjusted Classification of Congenital Heart Surgery (RACHS-1) scores, chronic disease, BPT, CCT, length in days of mechanical ventilation, ICU and hospital stay, and hospital mortality. Multivariate logistic regression with backwards stepwise elimination was used to determine independent associations. Cut off times were determined by ROC analysis (SPSS, 15.0, Chicago, IL). 
Results: During the study period, 230 children underwent cardiac surgery with 137 requiring both by-pass and cross clamping. These patients had a median (IQR) age of 0.8 years $(0.13-5)$, RACHS-1 score of 2 (2-3). ARF occurred in 20/137 (15\%) patients post-operatively. Compared to those who did not develop ARF, children who developed ARF had significantly longer median BPT [179 $\min (99-264)$ vs $104 \min (66-168)$; $\mathrm{p}<0.05$ ], CCT [ $80 \mathrm{~min}(55-157)$ vs $58 \mathrm{~min}(28-89) ; \mathrm{p}<0.05]$, and increased mortality [10\% $(2 / 20)$ vs $2 \%(2 / 117) ; \mathrm{p}<0.05]$. Of all variables measured, only BPT was independently associated with ARF. As a continuous variable, minutes of BPT were independently associated with ARF with an odds ratio (95\% CI) of 1.01 (1.01-1.02), $(\mathrm{p}=0.001)$. In other words, each minute on bypass increased the odds of developing ARF by $1 \%$. Alternatively, a $\mathrm{BPT}>180 \mathrm{~min}$ was also independently associated with ARF with an odds ratio of $2.97(1.09-8.16),(\mathrm{p}=0.034)$. A BPT $>200 \mathrm{~min}$ was independently associated with increased hospital mortality with an odds ratio of $15(1.5-150),(\mathrm{p}=0.02)$.

Conclusion: ARF is a significant problem following surgery for congenital heart disease in children and is associated with increased hospital mortality. In this population, by-pass times of $>180$ and $>200 \mathrm{~min}$ were independently associated with increased ARF and hospital mortality, respectively. These findings suggest that efforts to limit BPT may be important in improving outcomes in children undergoing surgery for congenital heart disease. However, larger studies are needed to better assess confounding factors.

Routine Developmental Follow-Up for Children with Complex Congenital Heart Disease: Preliminary Results from a New Clinical Program

Cheryl Brosig PhD, Kathleen Mussatto, Laurel Bear, Ann Chin

Medical College of Wisconsin, Children's Hospital of Wisconsin

Background: Children with complex congenital heart disease are at risk for developmental problems. To address this risk, in 2007, we created the Herma Heart Center Developmental Follow-Up Clinic. The clinic provides routine developmental assessment for children with complex congenital heart disease.

Methods: The clinic targets children with complex congenital heart disease who are under the age of 3 years. All children who have cyanotic lesions, who undergo surgery in the neonatal period (within the first 30 days of life), or children suspected to be at risk for developmental problems based on other factors (e.g. history of seizures, ECMO, complications during/after surgery, etc.) are eligible for the program.

Families are given information about the program prior to discharge from the hospital following surgery. The program coordinator contacts families to schedule appointments. The goal is to see children at 6 month intervals until the age of 3 years. Children older than 3 who have developmental problems are referred to the clinic's developmental psychologist for further evaluation.

The team consists of a nurse, developmental pediatrician, speech therapist, physical therapist, and occupational therapist. Each child is seen by all members of the team at each clinic visit. A standardized developmental assessment is performed using the Bayley Scales of Infant Development at each visit. Parents also complete the Infant Toddler Quality of Life Scale and the Impact on the Family Scale. Parents are given a developmental "report card" highlighting their child's strengths and weaknesses. Referrals to other specialists and referrals to early intervention services are made as needed.
Results: From January, 2007 through October, 2008, 162 visits were scheduled. 41 visits were cancelled due to illness or weather, and 13 visits were no shows, resulting in 108 clinic visits. 58 new patients were seen ( $54 \%$ of all visits). 32 patients were seen for multiple visits $(55 \%$ of new patients). $71 \%(n=41)$ were male and $29 \%(\mathrm{n}=17)$ were female. Race of the patients was as follows: $60 \%(\mathrm{n}=35)$ White, $17 \%(\mathrm{n}=10)$ Hispanic, $14 \%(\mathrm{n}=8)$ Black, $2 \%(\mathrm{n}=1)$ Asian and $7 \%(\mathrm{n}=4)$ other. The mean age was 12.92 months (range 6-38 months). Diagnoses included: Single right ventricle $(29 \%, \mathrm{n}=17)$; Transposition of the great arteries $(24 \%$, $\mathrm{n}=14)$; left ventricular outflow tract obstruction $(22 \%, \mathrm{n}=13)$; right ventricular outflow tract obstruction $(16 \%, \mathrm{n}=9) ; \mathrm{AV}$ valve abnormalities ( $5 \%, \mathrm{n}=3)$; cardiomyopathy $(2 \%, \mathrm{n}=1)$; and total anomalous pulmonary venous connection $(2 \%, \mathrm{n}=1) .47 \%$ $(\mathrm{n}=27)$ of patients were seeing multiple specialists in addition to cardiology. $64 \%(n=37)$ of patients had received or were receiving early intervention services (speech, occupational and/or physical therapy).

Conclusion: A routine developmental follow-up program is possible for children with CHD, although we have had some difficulty scheduling appointments around surgeries and illness. Overall, the program has been well-received by families, cardiologists and pediatricians, and we have plans to expand the program for 2009 and beyond.

\author{
Mechanism of Exercise Limitation in the Fontan: \\ A Prospective Assessment Using Supine and \\ Upright Exercise \\ Bryan Goldstein MD, Chad Connor MD, Lindsay Gooding, \\ George Stephens MS, Albert Rocchini MD \\ C.S. Mott Children's Hospital, University of Michigan, \\ Ann Arbor
}

Background: Patients palliated to Fontan physiology have reduced exercise capacity. Recent data suggest that limitations in the ability to augment stroke volume (SV) and pulmonary blood flow (PBF) are integral. It is not known whether PBF is limited by inadequate preload, excessive pulmonary vascular resistance (PVR), or both. In the normal subject, supine exercise, compared with upright exercise, elicits reduced exercise duration and peak oxygen consumption $\left(\mathrm{VO}_{2}\right)$ but is associated with an acute increase in venous return. If peak exercise PBF and exercise performance in the Fontan is predominately preload-limited, supine exercise would be expected to improve exercise capacity. We sought to determine whether supine exercise, compared with upright exercise, yields relatively improved exercise performance in patients with Fontan circulation, compared with healthy controls.

Methods: Fontan-palliated patients ages 8 to 30 years were identified and recruited, along with age- and sex-matched controls. Subjects were randomized to undergo either supine or upright graded bicycle ergometry with continuous ECG monitoring and breath-by-breath expiratory gas analysis. After a three hour rest period, all subjects crossed over into the alternate posture.

Results: 28 Fontan subjects (17 male, mean age $13.4 \mathrm{yrs})$ and 16 healthy controls ( 9 male, mean age $12.7 \mathrm{yrs}$ ) completed the protocol with no adverse events. The predominant anatomic diagnosis was HLHS $(n=9)$. Maximal exercise, as defined by a respiratory exchange ratio of $>1.09$, was achieved with upright testing in $22 \%$ of Fontan subjects and $80 \%$ of controls. Exercise performance was impaired in Fontan subjects, relative to control subjects, in both positions. The duration of exercise (in seconds, 
Supine: Fontan $312 \pm 67$ vs. Control $432 \pm 121, \mathrm{p}=0.0001$; Upright: Fontan $340 \pm 79$ vs. Control $470 \pm 134, \mathrm{p}=0.0002), \%$ predicted peak $\mathrm{VO}_{2}$ (Supine: Fontan $54.5 \pm 12.1 \%$ vs. Control $73.9 \pm 23.9 \%, \mathrm{p}=0.009$; Upright: Fontan $65 \pm 13.1 \%$ vs. Control $84.1 \pm 23.9 \%, \mathrm{p}=0.01$ ) and peak work (in Watts, Supine: Fontan $95 \pm 27$ vs. Control $120 \pm 29, \quad p=0.006$; Upright: Fontan $96 \pm 29$ vs. Control $121 \pm 36, \mathrm{p}=0.02$ ) were significantly greater in the control group, regardless of posture. The $\%$ predicted oxygen pulse $\left(\mathrm{O}_{2}\right.$ pulse; equals peak $\mathrm{VO}_{2} /$ peak HR), a surrogate for SV and PBF, did not differ between groups in either position, or between positions in either group. In both groups, exercise duration, $\%$ predicted peak $\mathrm{VO}_{2}, \%$ predicted peak HR, respiratory exchange ratio and breathing reserve were significantly greater in the upright position $(\mathrm{p}<0.05$ for all comparisons). The relative differences in $\%$ predicted peak $\mathrm{VO}_{2}, \%$ predicted $\mathrm{O}_{2}$ pulse and $\%$ predicted peak HR during upright and supine exercise did not differ between groups.

Conclusion: Supine exercise in Fontan-palliated patients results in the same relative reduction in exercise performance seen with healthy controls. PBF, as assessed by the $\mathrm{O}_{2}$ pulse, is not increased with supine exercise in the Fontan suggesting that pulmonary vascular tone, and not preload, is the limiting factor. Approaches aimed at reducing PVR to augment PBF, and thereby improve exercise capacity, ought to be explored.

Brain MRI Injury Before and After Neonatal Cardiac Surgery with High-Flow Bypass and Maximized Oxygen Delivery Strategy

Dean Andropoulos M.D., M.H.C.M., JV Hunter M.D., DP Nelson M.D., SA Stayer M.D., AR Stark M.D., ED McKenzie M.D., JS Heinle M.D., CD Fraser M.D. Jr.

Texas Children's Hospital/Baylor College of Medicine

Background: New intraparenchymal brain injury on MRI, consisting of white matter injury (WMI), infarction, or intraparenchymal hemorrhage, is observed in $36-73 \%$ of neonates after cardiac surgery with bypass (CPB). 1-3 We performed brain magnetic resonance imaging (MRI) before and after neonatal cardiac surgery, using high flow CPB protocol, hypothesizing that incidence of brain injury would be reduced compared to previous published studies, and to establish a baseline incidence for neuroprotection studies.

Methods: CPB protocol included $150 \mathrm{ml} / \mathrm{kg} / \mathrm{min}$ flows, $\mathrm{pH}$ stat management, hematocrit $>30 \%$, and high flow antegrade cerebral perfusion. 4 Regional brain oxygen saturation $\left(\mathrm{rSO}_{2}\right)<50 \%$ was treated by protocol. Brain MRI was performed preoperatively, 7 days postoperatively, and at age 3-6 months.

Results: 24 of 67 patients (36\%) had new postoperative WMI, infarction, or hemorrhage, and $15 \%$ had new WMI. (Table). Associations with new postoperative brain injury included preoperative brain injury $(\mathrm{p}<0.001)$, and low brain maturity score $(\mathrm{p}=0.05) .45$ patients had a 3 rd scan, with a $27 \%$ incidence of new minor lesions, but $58 \%$ of previous lesions had partially or completely resolved.

Conclusion: Despite an evidence-based strategy to maximize brain oxygen delivery in the perioperative period, and a reduction in incidence and severity of new injury and WMI compared to previous reports, there is still a significant incidence of both preand postoperative MRI abnormality in this population. Many lesions resolve in the first 6 months after surgery. Novel perioperative neuroprotective strategies are needed to assess whether long term improvement in neurodevelopmental outcomes can be achieved.
Table: MRI Data, Pre- and 7-Day Postoperative MRI Scans.

\begin{tabular}{|c|c|c|c|c|}
\hline Parameter & SV $n=36$ & $2 \mathrm{~V} \mathrm{n}=32$ & $\begin{array}{l}\text { P value, } \\
\text { SV vs. } 2 \text { V }\end{array}$ & Total \\
\hline $\begin{array}{l}\text { Preoperative WMI, } \\
\text { no., }(\%)\end{array}$ & $5(13)$ & $6(19)$ & 0.83 & $11(16)$ \\
\hline 7-day postoperative WMI, no., (\%) & $13(37) \#$ & $2(6)$ & $0.006 *$ & $15(22)$ \\
\hline $\begin{array}{l}\text { Preoperative infarct, } \\
\text { no., }(\%)\end{array}$ & $5(14)$ & $7(22)$ & 0.59 & $12(18)$ \\
\hline $\begin{array}{l}\text { 7-day postoperative infarct, } \\
\text { no., }(\%)\end{array}$ & $11(31)$ & $11(34)$ & 1 & $22(33)$ \\
\hline $\begin{array}{l}\text { Preoperative IP hemorrhage, } \\
\text { no., }(\%)\end{array}$ & 0 & 0 & 1 & 0 \\
\hline \multicolumn{5}{|c|}{ 7-day postoperative IP hemorrhage, no., (\%) } \\
\hline & $3(9)$ & $2(6)$ & 1 & $5(8)$ \\
\hline New 7-day postoperative WMI & $8(23)$ & $2(6)$ & 0.09 & $10(15)$ \\
\hline \multicolumn{5}{|c|}{ New 7-day postoperative WMI, infarct, or IP hemorrhage, no., (\%) } \\
\hline & $16(45)$ & $8(25)$ & 0.13 & $24(36)$ \\
\hline MRI Total Maturity Score (TMS) & $11.8 \pm 1.5$ & $11.8 \pm 1.0$ & 0.93 & $11.8 \pm 1.3$ \\
\hline Patients with TMS <; 10, no., (\%) & $8(22)$ & $6(19)$ & 0.96 & $13(21)$ \\
\hline $\begin{array}{l}\text { Preoperative total MRI injury } \\
\text { score }\end{array}$ & $1.8 \pm 2.6$ & $2.3 \pm 2.8$ & 0.47 & $2.0 \pm 2.7$ \\
\hline \multicolumn{5}{|c|}{ 7-day postoperative total MRI abnormality score } \\
\hline & $3.8 \pm 4.2 \#$ & $3.2 \pm 3.7$ & 0.57 & $3.5 \pm 3.9^{\wedge}$ \\
\hline \multicolumn{5}{|c|}{ Pre/postop change in total MRI abnormality score } \\
\hline & $1.9 \pm 3.2$ & $0.9 \pm 3.2$ & 0.22 & $1.5 \pm 3.2$ \\
\hline
\end{tabular}

Data expressed as numbers of patients and percentages for MRI findings, and mean \pm SD for numerical scores.

${ }^{*} \mathrm{p}<0.05$ for between group differences. ${ }^{*} \mathrm{p}<0.05$ for pre- to postoperative difference within groups. ${ }^{\wedge} \mathrm{p}<0.05$ for pre- to postoperative difference for both groups combined. WMI, white matter injury; IP, intraparenchymal.

Brain Immaturity on MRI is Associated with Pre- and Postoperative Brain Injury in Neonates Undergoing Cardiac Surgery

Dean Andropoulos M.D., M.H.C.M., JV Hunter M.D., DP Nelson

M.D., SA Stayer M.D., AR Stark M.D., ED McKenzie M.D.,

JS Heinle M.D., CD Fraser, M.D. Jr.

Texas Children's Hospital/Baylor College of Medicine

Background: Full-term neonates with congenital heart disease have brain immaturity on MRI scans, including delays in both structural and microcellular development.(1,2) Brain immaturity is a well established contributing factor to white matter injury in premature neonates, and may explain the vulnerability of full term neonates with $\mathrm{CHD}$ to brain injury. We hypothesized that neonates with brain immaturity are more likely to have both preand postoperative brain injury.

Methods: 68 neonates with CHD, 35+ weeks gestational age, had pre-, and 7-day postoperative MRI. Brain abnormality score was calculated, including white matter injury (WMI), infarction, and hemorrhage. Brain maturity was assessed from T1 and T2weighted MRI using the Total Maturity Score (TMS) of Childs et al. (3) scoring 4 features: myelination, cortical infolding, germinal matrix, and bands of migrating glial cells.

Results: Birthweight was lower in single ventricle vs. two ventricle patients $(3.01 \pm 0.33$ vs. $3.27 \pm 0.54, p=0.02)$, but there was no difference in gestational age $(38.3 \pm 1.2$ vs. $38.8 \pm 1.3, \mathrm{p}=0.10)$, head circumference $(33.3 \pm 1.6$ vs. $33.8 \pm 1.3, \mathrm{p}=0.11)$, or TMS $(11.8 \pm 1.5$ vs. $11.8 \pm 1.0, \mathrm{p}=0.93)$, or number of patients with TMS <; $10(22 \%$ vs. $19 \%, \mathrm{p}=0.96)$. However, seven of 13 patients with TMS $<$; 10 (equal to brain maturity of 35 weeks gestational age or less) had a postoperative brain injury score of 6 or above (moderate injury), vs. only 10 of 54 with TMS $>10$. $(\mathrm{p}=0.01)$. Preoperative WMI was more likely with TMS $<10(5 /$ 13 vs. $6 / 55, \mathrm{p}=0.03)$, as was postoperative WMI (6/13 vs. 9/54, $\mathrm{p}=0.05)$.

Conclusions: Neonates with CHD who have a structurally immature brain are more likely to have both pre- and postoperative WMI, as well as a moderate or severe postoperative brain injury score. This could explain the high rate of WMI in these patients, as the brain 
in CHD shares features with the premature neonatal brain, which also has a high rate of WMI.

Beads of Courage in the Cardiac Population

Christine Langston RN, BSN, Wendy Pauker, Jean Baruch, Cristy Puetz

Phoenix Childrens Hospital

Background: We have utilized the beads of courage program in the cardiac ICU to facilitate the patient/parents difficult journey through cardiac surgery from diagnosis to recovery. The beads represent a piece of the journey that the family/patient can have as a tangible momentum of their journey. The program has been found to assist with resilience, and the anticipation of "earning" a new bead alleviates some of the stress and fear of treatment. The staff finds that the beads of courage program is a tool that helps to build rapport with the patient/family, and is something that can be done FOR the patient and not to the patient.

Methods: Satisfaction survey. Participants and nurses involved were directly surveyed.

Results: At this point, there is $100 \%$ satisfaction among the families/ patients involved in the program. Satisfaction is lower among staff. Conclusion: The beads of courage program has been a positive influence in the cardiac ICU. Families become invested in the program and seek out staff to collect the beads that their child has earned. They find the beads to be an important and tangible item that remains important to them, and it remains a connection to their child in the event of the death of the patient. The nurses find that the program is a factor in practicing the art of nursing.

\section{RN Career Planning - Novice to Expert in the midst of a 'War for Talent' \\ Alison Dodds RN, BScN, Karen Kinnear RN, BScN, MBA The Hospital for Sick Children}

Background: During the current nursing and health care professional shortage, retention of experienced, knowledgeable professionals is critical to the success of any healthcare institution. Current predictions are that by 2020 the number of available nurses will be $20 \%$ below projected needs, a shortage that threatens to cripple the healthcare system (Buerhaus, Staiger \& Auerbach, 2000). Critical care nurses are special, they have specialist skills that organisations need to nurture and reward and recognise. Retention and recruitment of this population is essential if we are going to continue to provide expert care for our critical care patients. But the war for talent has begun and the generational gap needs to be addressed. These two factors need to be incorporated into an organizations strategy for recruitment and retention. The career advancement framework created at Sick Kids was build from a needs assessment of staff and literature regarding the generational differences and the 'war for talent'.

Methods: From December 2006 through to April 2007 there was a total of 9 focus group sessions conducted throughout Sick Kids which involved nurses and professional services. The focus group sessions were focused around the concept of career advancement and what the participants felt the organisations' and the individuals' responsibilities were.

Results: The focus group results were grouped under the key themes of: Professional Development, Career Opportunities, Career Pathways and Reward and Recognition.

Conclusion: In conclusion the different generational needs as well as the outcomes from the focus group sessions were combined to develop an online career advancement tool named SCOPE (Strategic Career Opportunities for Professional Development). This presentation will describe the program content and the framework used.

\section{Mentorship Program for Critical Care \\ Alison Dodds RN, BScN \\ The Hospital for Sick Children}

Background: The mentorship program was created to provide a "structured" support system for nurses and Respiratory Therapists beyond the preceptorship program within the Critical Care Unit. The Focus is on both novice and experienced RNs and RRTs. The clinical and leadership staff within the CCU and PICU identified the need for a mentorship program in 2006-2007 during a significant recruitment and retention issue within the nursing department. Retention and recruitment of experienced and knowledgeable RNs and RRTs is of critical importance to the success of a health care institution. A recent study by Kilty (2006) indicated that nurses who participated in a mentorship program were more likely to remain within the organisation as they felt that they had gained more confidence and their transition into the work environment was less stressful. The leadership team decided to implement an interprofessional mentorship program rather than a traditional intraprofessional program, which aligns with the hospitals' strategic direction to advance inter-professional practice within the organisation. Further more, in an environment where RNs and RRTs rely heavily on each other for day to day operations it made more sense to run as one inter-professional program.

Methods: Aims:

- To provide a common framework of mentorship for RNs and RTTs within the Critical Care Unit (CCU).

- To improve retention of nurses within the CCU over a period of 2 year.

- To improve the recruitment of nurses and RTs within the CCU over a period of 2 years.

- To increase staff satisfaction within the Critical Care Unit over a period of 2 years.

A mentorship framework was created after an extensive literature search and benchmarking of other institutions. Focus group sessions were conducted for mentors, mentees and new staff who had entered into a mentorship relationship to evaluate the program.

Results: The results from the focus group sessions were inserted into the mentorship program and will be discussed.

Conclusion: To date we have a successful mentorship program which has had a total of 91 staff participating in the training and a total of 22 dyads over this period of time with two triads. This presentation will describe the program content and the framework used.

MRI Brain Scans can be Performed Safely, and Without Anaesthesia, in Young Infants with Critical Heart Disease Michelle Goldsworthy BN, J Gunn, R Hunt, C Delzoppo, J Beca, $L$ Whelan, T Inder, L Shekerdemian

Royal Children's Hospital, Melbourne, Australia and Starship Children's Hospital, Auckland, New Zealand

Background: Magnetic Resonance Imaging (MRI) is now considered to be the 'gold standard' investigation for the early identification of brain injury in high-risk infants with congenital heart disease (CHD). Many clinicians and researchers consider that a relative shortcoming of MRI, is the requirement for a general 
anaesthetic in order to complete the procedure and yield goodquality images. At the Royal Children's Hospital, Melbourne, we have for the past 8 years performed MRI scans on unanaesthetised infants aged up to 3 months, without additional sedation, both for clinical indications and within the context of prospective research studies.

The Hearts and Minds Study is a prospective study of brain injury in very young infants undergoing surgery for congenital heart disease. The protocol includes three MRI scans: preoperatively, at around one week after surgery, and at around 3 months of age. We present here our experience with performing MRI scans in our cohort of study participants.

Methods: Seventy five infants undergoing surgery for CHD at 8 weeks of age or less were recruited to the study, 68 of these infants received preoperative scans. Of these, $39(57 \%)$ were receiving prostaglandin infusions of which 29 had duct dependent systemic circulations. MRI scans were performed on a Siemens $1.5 \mathrm{~T}$ or $3 \mathrm{~T}$ Scanner, with sequences including conventional and diffusionweighted imaging, tensor imaging and spectroscopy. Thirteen (19\%) participants were already intubated and sedated, and in the Paediatric Intensive Care Unit (PICU) at the time of the preoperative MRI scan. All other pre-operative, and post-operative scans were carried out without anaesthesia. For the purpose of the study, non-anaesthetised infants were fed (where appropriate), wrapped in a blanket, and placed in a vacuum fixation device. Intravenous infusions of inotropes, dextrose and prostaglandin were continued during the MRI scan. Infusion pumps, and tubing were all changed to MRI compatible equipment prior to leaving the in-patient environment. Intubated infants were transitioned to the MRI-compatible transport ventilator, and observed for a 10-15 minute period, prior to leaving PICU.

Results: 182 MRI scans were performed in this cohort, of which 169 were without any anaesthesia or sedation. 43 of these were performed as out-patients. The scan duration was $25-45$ minutes. MRI scans proceeded uneventfully, without any evidence of clinical instability during the imaging period or during patient transport to or from the scanner. Only one of the scans was rendered uninterpretable due to movement in one non-intubated patient. No adverse events occurred during the MRI scans, though two events occurred shortly afterwards. One participant was hypoglycemic (glucose $1.8 \mathrm{mmol} / \mathrm{l}$ ) on return from scan, the dextrose infusion had become disconnected. This was treated with an appropriate increase in glucose infusion rate. Another was noted to be cool on return to the ward and over head heating was recommenced.

Conclusion: MRI scans can safely be carried out, without anaesthesia, in young infants with critical heart disease. We would suggest that general anaesthesia is not specifically indicated for MRI scans in this age-group, and consideration should be given to utilising this approach more widely.

Functional Outcomes in Patients with Congenitally Corrected Transposition of the Great Arteries

Michael Gaies MD, MPH, CS Watnick, JG Gurney, EL Bove, CS Goldberg

University of Michigan

Background: Physicians caring for children with congenitally corrected transposition of the great arteries (CCTGA) must choose between multiple surgical and medical treatment options. Anatomic repair is being used with increased frequency based on the intent to improve long-term functional outcomes. It is therefore necessary to assess the quality of life (QOL) and functional status in patients with anatomically-repaired CCTGA as they move into late childhood and adulthood.

Methods: All patients born after 1983 and treated at our institution for CCTGA, 45 with anatomic repairs (AR) and 18 with either conventional repair or no surgical procedure (non-anatomic repair, NAR), were contacted to participate. Subjects completed the PedsQLTM 4.0 Generic Core Scale and 3.0 Cardiac Module parentproxy (for ages 2-18) and self-report forms (for ages 8-25). QOL scores were calculated for total function and for individual functional domains on each module. For the Generic Scale, the psychosocial summary score was calculated from the emotional, social, and school functioning scores. Responses were linearly transformed so that higher scores indicate better QOL, with 100 as the maximum. Univariate analyses were conducted for each domain, and t-tests were used to test mean differences in response between the AR and NAR groups, and to measure the impact of potential risk factors for lower QOL in the AR subgroup.

Results: QOL instruments were completed by 51/63 (81\%) eligible patients, 38 of whom (75\%) underwent AR. Mean age at followup was $7.3 \pm 4.3$ years in the AR group vs. $14.3 \pm 4.5$ in the NAR group and the mean duration since the time of AR was 68 months. Caregivers of AR patients reported similar quality of life scores compared to the NAR group for total QOL (78 vs 73), physical function (82 vs. 77), and psychosocial function ( 75 vs. 71 : $\mathrm{p}>0.05$ for all measures). The NAR group's self-reported school function score was substantially higher ( 81 vs. $63, \mathrm{p}=0.021$ ) than the AR self-reported score, but self-reported scores in the other domains were similar. The parent-reported school functioning scores were similar. On the Cardiac Module, AR patients self-reported fewer problems related to residual heart disease ( 75 vs. 63 ), physical appearance ( 81 vs 68 ), and treatment anxiety (74 vs. 59), although the differences were not statistically significant. Among AR patients with a hospital stay $>21$ days, parents reported lower QOL related to residual heart disease (67 vs. $81, \mathrm{p}=0.03)$, treatment anxiety ( 52 vs $73, \mathrm{p}=0.06)$, and communication ( 47 vs.71, p $=0.06$ ) compared to those who stayed $<21$ days.

Conclusion: At intermediate follow-up, patients who underwent AR for CCTGA reported similar QOL and functional status compared to NAR patients, though the data suggest lower school functioning in AR patients. Prolonged hospital stay after AR may be a risk factor for lower QOL. Repeated QOL assessment into adulthood is necessary to determine the comparative benefits of AR. Given the rarity of this disease, collaboration across centers is necessary to answer these important questions.

\section{Caring for Chronic Patients in the Cardiac Critical Care Unit Cara Dostal RN, Elena Nikolsky RN \\ The Hospital for Sick Children}

Background: As medicine advances with regards to pediatric cardiology, more patients are viable for technological support and surgical interventions that prolong their life expectancy. Due to acute nature of their illnesses some clients spend extremely long periods of time in the Cardiac Critical Care Units. Since coping strategies and emotional reactions greatly differ between the families, some parents might experience a situational crisis related to their child's illness and hospitalization. Therefore, effective communication and an adequate support are imperative to meet the growing need for efficient care of acutely ill long stay patients and their families. This presentation addresses concerns that were brought by Cardiac Critical Care staff and families of acutely ill long term patients at the Hospital for Sick Children. In the year 
2008 acute/ chronic patients represented $30 \%$ of CCU population. Some issues that have arisen incorporated:

- Tensions between staff caring for the patients and the families around treatment plans

- Challenges experienced by CCU staff due to the tension

- Families frustration with medical and nursing consistency over the care for acutely ill long term patients in the teaching institution

Methods: To address staff's and families' concerns and minimize negative outcomes the Cardiac Critical Care team implemented support structures and came with some recommendations

1) Introduced a Cardiac Nurse Practitioner to follow the family and patient's progress for one month at a time.

2) Established a core group of nurses to be assigned to the patient $25 \%$ of the time.

3) Institute early support network to follow each family within first two weeks of admission to CCCU.

4) Support core group of nurses with opportunities for debriefing and 'breaks' from the coring.

5) Encourage family communication through multidisciplinary meetings. Have interpreters available.

6) Introduce a core CCU fellow and Respiratory therapist to achieve consistency.

Results: Patients' families have indicated that:

Introducing the role of Nurse Practitioner in the unit created a valuable recourse to follow trends and achieve medical stability.

A core cardiac interdisciplinary team allowed consistency when following up on changes in condition and advocating for the patients and families.

Multidisciplinary meetings helped to facilitate discussions regarding patients' status and future treatments plans.

Social support network assisted to overcome multiple financial and emotional issues related to long term hospitalization.

The Cardiac Critical Care staff supported formation of a core list to achieve care consistency and trust development. However, practitioners expressed concerns regarding the staff's burnout while caring for acute long-term patients more than $25 \%$ of the time. Consistent family meetings to evaluate patient progress, is revered as a need by both family and staff.

Conclusion: A literature search was conducted and outlined that more research is needed surrounding the care of chronic cardiac critical care patients in the CCCU. Strategies must be implemented as soon as possible to minimize stress and anxiety surrounding care delivery. Communication is the key in developing trust relationships between staff and family. Outlets for staff and family to reveal frustrations are viewed as being very positive by both families and staff.

Effectiveness of Chern En Chiang ECG algorithm for determining location of Wolff Parkinson White pathway in the pediatric population

Jamie Ganley RN, Lisa Gervasi, MSN

The Children's Hospital of Philadelphia

Background: Wolff Parkinson White (WPW) is a syndrome in which there is an extra conduction pathway from the atrium to the ventricles, which may cause tachyarrhythmias. The electrical impulse goes to the ventricle prematurely, causing a short PR interval and a slurred onset of the QRS (delta wave). WPW pathways can be located on either the right or left side of the heart, anywhere along the atrioventricular groove. There are several established ECG algorithms available to determine locations of WPW pathways in the adult population. The Chern En Chiang algorithm is commonly used in the electrophysiology lab at the Children's Hospital of Philadelphia (CHOP). However, there is little research on whether this algorithm is effective in the pediatric population. Determining the pathway location prior to the EP study can be useful to the staff involved in the study and to the family, as supplies and risks differ based on the pathway location. This study examined ECGs to determine whether the Chern En Chiang algorithm is effective in predicting WPW pathway locations in the pediatric population.

Methods: This is a retrospective analysis of all patients aged 0-19 with the diagnosis of single WPW pathway that underwent ablation procedures from 1/1/2004-6/30/2008 at CHOP. Baseline ECG's were analyzed and applied to the algorithm. After determining from the algorithm where the suspected pathway would occur, electrophysiology reports were then studied to determine the actual location of the pathway.

Results: 172 patients qualified for this study. The average age for patients in this study was 13.52 years. The Chern En Chiang algorithm was successful at predicting WPW pathway location in 93\% of our patients, which is the same as with adult patients. Predicted locations of right sided pathways were the same or more accurate as the adult study. Predicted left sided pathways were the same or less accurate than the adult study. However, all predicted left sided pathways were truly on the left side of the heart, so the risks associated with being on the left side of the heart would remain.

Conclusion: The Chern En Chiang algorithm is as effective in the pediatric population as it is in the adult population in determining WPW pathway locations.

Transitioning Pediatric Patients from Intravenous Epoprostenol to Subcutaneous Treprostinil: Model of a Pediatric Pulmonary Hypertension Service

Jaclyn Sawyer PharmD, Michelle Bagby RN, Russel Hirsch MD

Cincinnati Children's Hospital Medical Center

Background: Pulmonary Hypertension is a chronic and complex disease with evolving treatment options. Epoprostenol and treprostinil are approved continuous infusion prostanoids that produce direct vasodilation of the pulmonary and systemic vascular beds, and inhibit platelet aggregation. Differences between these products are related to chemical stability, pharmacokinetics and route of administration. Thus, the rationale for transitioning patients stable on epoprostenol to treprostinil is solely for improved quality of life. However, the best practice when transitioning pediatric patients onto alternative therapies is unclear. Lack of standardization and inappropriate planning can lead to disjointed medical care and suboptimal patient outcomes. We present our experience with conversion of a 14 year old patient from an intravenous to a subcutaneous form of prostanoid, and discuss planning and logistic considerations.

Methods: The patient had been diagnosed with idiopathic pulmonary hypertension 3 years prior to this admission, and maintained on continuous intravenous prostacyclin from the time of diagnosis. The model for this transition was developed within our pulmonary hypertension service using a critical review of our practices, advice from other pediatric institutions, input from manufacturer representatives and discussion with the patient and family. The goal was a safe and efficient transition to subcutaneous treprostinil. Appropriate hospital administrative approval was 
obtained. Using a detailed simultaneous titration of increasing treprostinil while decreasing epoprostenol, as outlined by previous studies, a safe transition was achieved in an intensive care setting. Total admission time was less than 30 hours.

Results: Our model of care has resulted in standardization of a complex transition. It eliminates medical errors and miscommunication, while still allowing individualization of therapy. This model allows for anticipatory, rather than reactionary decision making. It will promote mainstreaming treatment, and improved patient quality of life and quality of care. Technical complications in our model were limited, and could be attributed to the education, preparation and support by the healthcare team. Clinical complications were limited to site pain, requiring local measures for support.

Conclusion: This model of comprehensive and collaborative pediatric medical care resulted in improved patient outcomes and quality of life. Alternative approved medical therapies for critical conditions can be transitioned in an efficient and safe manner when a proper and thorough plan is implemented.

Oral Feeding Outcomes for Infants with Congenital Heart Disease

Sharon Sables-Baus RN, MPA, MS, PhD, Debra Paul OTR, Jon Kaufman MD, Eduardo daCruz MD

The Children's Hospital, Denver, CO

Background: Infants with congenital heart disease (CHD) are at significant risk for somatic growth failure and developmental delay. This is due to multiple factors. Although overall surgical outcomes have improved, perhaps early timing of surgical repair or palliation, the peri-operative course itself or medically necessary interventions place the infant at risk for growth and development delay. Determination of factors that impact oral feeding skills is difficult; however, challenges to oral feeding begin in the hospital and continue after discharge. The purpose of this study was to examine factors that may influence oral feeding progress for infants with CHD who required surgical repair as neonates.

Methods: A retrospective chart review of 55 neonates, (greater than 35 weeks gestation and less than 1 month of age) with congenital heart disease (CHD) admitted to Cardiac Intensive Care Unit (CICU) at The Children's Hospital Colorado from January 2006-December 2007 and who underwent surgical intervention. We sought to identify clinical variables that would predict success or failure in achieving full oral feeding. Variables examined included length of stay, duration of endotracheal intubation, dependence on continuous positive airway pressure (CPAP or BiPAP), duration of circulatory arrest, aortic cross clamp time, and cardiopulmonary by-pass time. We also examined interventions such as occupational, speech and lactation consults, as well as maternal desire to breast feed at time of admission as predictors of successful oral feeding at time of discharge. Primary data analyses were completed in HLM 6.0 (Raudenbush, Bryk, \& Congdon, 2006), with feeding episodes, breathing methods, and time since surgery modeled as a within-person (random) variables and infant's demographic, clinical, and treatment episode characteristics modeled as between-person (fixed) factors. Additional descriptive analyses, correlations, and psychometric calculations were performed in SPSS 16.0.

Results: Post-operatively, twenty four infants (44\%) failed to achieve full oral feeds at discharge. Factors found to be significant predictors: length of stay, days of mechanical ventilation, need for CPAP, by-pass time, cross clamp time and circulatory arrest. All with p-values $<.05$. Intra-operative trans-esophageal echocardiography was not found to be a significant risk factor for inability to achieve full oral feeds. The 24 infants were all discharged with an in-dwelling naso-gastric feeding tube. Initiation of occupational, speech, or lactation support did not influence infant's ability to achieve full oral feedings at discharge. Although all mothers met with lactation upon admission, only 13 mothers (24\%) attempted to breastfeed during the hospitalization and none of the infants were discharged breastfeeding. Conclusions: Almost half of infants with CHD that required surgical intervention during the neonatal period are unable to achieve full oral feeds at discharge. This may be a significant cause of morbidity and likely affects somatic growth, cognitive and social development and parental confidence. None of the infants studied were discharged home fully breastfeeding. In a time when many benefits of breastfeeding are well known, this is concerning. The optimal methods for support of oral feeding progression in this population have yet to be established. Specific feeding management strategies based on scientific rationale are needed.

\section{Impact of a Unique Statewide Fetal Cardiology Program on Birth Location of the Norwood Candidate over a 12-Year Period Amy Svenson MD, Robin R. Fountain-Dommer, Donna Johnson, Scott M. Bradley, Anthony Hlavacek, Andrew M. Atz, Benjamin Horne, C. Osborne Shuler, Charles Trant, J. Philip Saul, Girish Shirali Medical University of South Carolina}

Background: In 1999, pediatric cardiologists and perinatologists in South Carolina joined together to develop a model program for providing cardiac services to children with congenital heart disease. The aim of this program was to enhance care and outcomes for these patients, in part by centralizing the births of all prenatally diagnosed cases of critical heart disease to a single tertiary care center (TCC). In our state, this tertiary care center is also the only center statewide where cardiac intervention and surgery for critical congenital heart disease is performed. In the current study, we sought to assess the success of this program by examining the birth location (tertiary care center versus elsewhere) of neonates with a functionally single ventricle and obstruction to the systemic outflow tract (Norwood candidates).

Methods: We studied all fetuses or newborns identified by echocardiography at our institution to be Norwood candidates between October 1995 and December 2007. A retrospective chart review was performed on those patients who met criteria to determine location of birth and prenatal diagnosis. Patients were then grouped by year of birth, and the percentage of patients born at the tertiary care center versus elsewhere was calculated for each year. We then compared the birth years prior to starting the statewide program (1995-1999) to those afterward (2000-2007), using a T-test.

Results: Over the 12-year study period, 181 Norwood candidates were identified. Grouped by year of birth, results are as follows: 1995,1 of $4(25 \%)$ candidates was born at the TCC; 1996, 2 of 11 $(18 \%)$ candidates were born at the TCC; 1997,1 of $7(14 \%)$ candidates was born at the TCC; 1998, 2 of $14(14 \%)$ candidates were born at the TCC; 1999, 8 of $16(50 \%)$ were born at the TCC; 2000,6 of $16(38 \%)$ were born at the TCC; 2001,10 of $18(56 \%)$ were born at the TCC; 2002, 9 of $17(53 \%)$ were born at the TCC; 2003,8 of $16(50 \%)$ were born at the TCC; 2004,16 of $23(70 \%)$ were born at the TCC; 2005,5 of $13(39 \%)$ were born at the TCC; 2006, 7 of $16(44 \%)$ were born at the TCC; 2007,9 of $10(90 \%)$ were born at the TCC. After institution of the statewide program, the percentage of Norwood candidates born at the TCC versus elsewhere increased significantly (24\% for $1994-1999$ vs. $55 \%$ for 2000-2007, $\mathrm{p}=0.008$ ). 
Conclusion: The statewide program has resulted in a statistically significant increase in the rates of Norwood candidates born at our tertiary care center over time $(\mathrm{p}=0.008)$. Centralization of care of complex congenital heart defects may have important implications for the uniformity of pre-operative management.

The Bedside View of Family-Nurse Communication: An Evidence-Based Review of Best Practices Erin Forster RN, Ashley Patton RN, Jennifer Poserina RN, J-S Rim RN, Mary Stuart RN, Michele Ulrich RN, Christina Walden $R N$, Sharon Barton RN PhD

The Children's Hospital of Philadelphia

Background: Nurses have a unique role in facilitating information sharing between patients, families and the interdisciplinary patient care team. Lapses in communication have been associated with hospital errors. Clear communication between nurses and families can improve patient safety, increase adherence to the plan of care, decrease patient length of stay, and increase family satisfaction. In our institution, communication between nurses and families has not been optimal. A plan of care communication tool has been developed, but has not been validated. A review of current practice indicates usage of $90 \%$ in our ICU and $10 \%$ in our step down unit, with a clear decrease noted on nights and weekends. Our current tool in the ICU does not allow for family input. Our step down unit's tool allows for family input, but is not consistently utilized.

Methods: Granger and Chulay's rating worksheet was used to prioritize research questions for further study. Staff scored familynurse communication as a top choice for investigation. Using the IOWA model of evidence-based practice, the research committee began the process of searching, reviewing, and grading existing evidence for best practices in family-nurse communication about the plan of care. Summary tools developed by Johns Hopkins served as our framework for summarizing and grading research and non-research evidence.

Results: Forty articles were reviewed investigating the question "what is the best practice for communication between nurses and families about the plan of care?" Of those 40, the strength of evidence was scored from 1 to 5 . A score of 1 is a well-designed randomized controlled trial while a score of 5 is evidence based on opinion. Two articles were at score 1,4 at score 2,1 at score $2-3$, 20 at score 3,7 at score 4,2 at score $4-5$, and 4 at score 5 . The Johns-Hopkins scoring model assigns quality grades ranging from A-D with $\mathrm{A}$ being highest quality and $\mathrm{D}$ indicating lowest quality. Of the 40 articles, 15 were A quality, $4 \mathrm{~A}-\mathrm{B}$ quality, and 21 B quality.

Literature reviewed did not identify one best practice, but recommended that practice should include: an effective tool for information sharing with patients and families, parent participation in rounds, clear and transparent information, and maintaining informational continuity despite shift change. Best communication practices included asking parents their opinions and for questions, frequent updates about the plan of care, using a shared decision making model, striving for consistency, empathy and listening.

Conclusion: As family-centered models of bedside care evolve, nursing evaluates our process of keeping families informed in order to deliver safe patient care and improve family satisfaction. Next steps for our group of bedside nurses are to complete a staff and family communication survey and validate and test a bedside communication tool.
Nutritional Status and Short Term Surgical Outcome in Children with a Single Ventricle Undergoing Fontan Completion

Martha Willis RN, MS, CPNP, Mary Pat Alfaro, RD, MEd, Jeffrey Anderson, MD, MEd Catherine Krawczeski, MD, Peter Manning, MD Cincinnati Children's Hospital Medical Center

Background: Children with single ventricle physiology are often small and at risk for failure to thrive. Malnutrition is particularly prevalent in this population. It is known that malnutrition in hospitalized patients leads to increased complication rates, increased length of hospital stay and increased hospital costs. Research shows that infants with a single ventricle and failure to thrive have worse short-term surgical outcomes at their second stage surgical repair. There is little known about the relationship between failure to thrive, nutritional management, and surgical outcome at the time of the Fontan procedure. With this knowledge we have recently developed and implemented guidelines in an attempt to improve nutrition and outcomes in our population of children undergoing the Fontan procedure.

Methods: Our guidelines include speaking to the patient's caregivers about a month prior to surgery and encouraging them to give their children a high calorie diet and a daily multivitamin with zinc. Postoperatively, we are providing the children with enteral feeds until oral intake improves. Enteral feeds, via a nasogastric tube which is placed in the operating room, are started the first postoperative day and increased as the patient tolerates. We complete a nutritional assessment form and implement intravenous supplementation based on tolerance of enteral feeds to achieve adequate caloric intake. We reviewed Fontan patient charts from the previous two years and compared the average number of days to goal intake, length of stay and infection rates to the patients who received the nutritional guidelines.

Results: We reviewed 17 patient's charts prior to the implementation of our guideline. Only 11 patients had nutritional assessment forms. Nutrition goal intake was defined as $70 \mathrm{Kcal} / \mathrm{kg} .7$ patients met nutrition goal intake before discharge. It took the patients an average of 6.9 days to meet the goal. Their average length of stay was 13.7 days, and 3 patients had wound infections during their hospitalization. When comparing these results to the patients that received nutritional education pre-operatviely and more aggressive enteral feedings, we found that all 13 patients (April 2008November 2008) had a nutritional assessment form. Caloric goal intake was achieved in 3.25 days which is a decrease of 3.65 days. The average length of stay is 9 days. This is 4.7 days less than prior to the implementation of our guidelines. Finally, there were no wound infections.

Conclusion: While these guidelines were recently implemented, the preliminary results show that diligent nutritional assessment and better pre-and post-operative nutrition can improve outcomes in the patients receiving the Fontan procedure. With continued implementation of these guidelines post-operative patients will achieve their caloric goals more rapidly. This will result in continued decreased length of stay and wound infection rates.

\section{Beyond the Building of Hybrid Suites; The Hybrid Nurse Staffing Model Terri Miller RN, Karen Krusinski Akron Children's Hospital}

Background: The Heart Center at Akron Children's Hospital in Akron, Ohio is a de novo program started in July, 2001. It is a unified team of cardiologists and surgeons under one single 
administrative structure, going beyond traditional interdisciplinary care provided between different departments. This team works to diagnose, treat and provide follow-up care to complex patients in a noncompetitive manner. In order to deepen this approach, we developed one nursing team for our operating room and cath lab. Methods: Our nursing staffing model is unique in many ways. Our heart center does approximately 250 cases in the cath lab and 125 surgeries. Our team consists of three registered nurses, three radiology technicians, and two surgical technicians. The registered nurses' responsibilities include pre-surgical assessments for outpatients, circulating in the operating room as well as the cath lab which include EP and interventional cases, and scrubbing in the operating room. A typical weekly assignment for the nurse includes two days in the operating room, one day in the cath lab, with one nurse being assigned to the extra cath lab day each week. The nurses' responsibility for call includes both the operating room and the cath lab therefore only requiring one call team. For training and orientation, a new employee is assigned to a mentor. The new orient follows the mentor for approximately a six month period. The focus begins with circulating, first in the cath lab then moving to the operating room. Finally, the scrub role in the OR is introduced. A successfully oriented nurse is able to fill each of these roles.

Results: The OR nurse is not just a visitor in the cath lab, tiptoeing back and forth, but an actual member of one unified nursing team that is interchangeable. The knowledge base of this hybrid nurse not only includes the anatomy of congenital cardiac defects and repairs, but also the physiology and electrophysiology of our complex patients. This increases the nursing knowledge to a 3dimentional view. Staffing is more efficient because the nurses staff both operating room and cath lab decreasing staffing costs. This staffing model provides a savings of $\$ 125,000$ per year by not needing two additional full time nurses. The hybrid nurse will save approximately $\$ 11,232$ per year in call pay. The greatest benefit for the patient is continuity of care. Frequently, the same nurse provides care for a patient who has both a diagnostic cath followed by a surgical repair. Being familiar with the needs of both the patient and the surgeons, benefits the team by anticipating the supplies and equipment needed for the case.

Conclusion: As more and more congenital cardiac cases require interplay between cath labs and operating rooms, our hybrid nurses will easily adapt to this trend. With rising costs in healthcare, our cost savings in our staffing model will help us allocate these monies to other needed areas. Finally the hybrid nurse staffing model contributes to the continuity of care of the patient as well as the expertise of our unique cardiac team.

\section{Attitude Toward and Current Practice of Transfer and Transition of Adolescents with Congenital Heart Disease in the United States of America and Europe \\ Philip Moons PhD, RN, Deborah Hilderson, Kristien Van Deyk, Amy Verstappen, Adrienne Kovacs, Susan Fernandes, Mary M Canobbio, Desiree Fleck, Alison Meadows, Rachel Linstead, Arwa Saidi Katholieke Universiteit Leuven, Belgium}

Background: More than $90 \%$ of children with congenital heart disease survive into adulthood. This has resulted in an increased awareness of the importance of coordinated processes to transition and transfer adolescents and young adults from pediatrics to adultfocused health care. Moreover, the 32nd Bethesda Conference of the American College of Cardiology and other international recommendations recognized the need to prepare the transfer of patients with congenital heart disease with a structured transition plan. To date, it is not known to what extend pediatric cardiology programs have implemented these international guidelines. The aims of this study were to describe the current practice and to explore the attitudes of pediatric cardiologists in the United States of America and Europe on transfer and transition of children with congenital heart disease.

Methods: An international survey among the chief pediatric cardiologists in the US and Europe was undertaken. Seventy-two centers completed and returned the 61-item questionnaire, which was specifically devised for this survey.

Results: Of 72 centers, $73.6 \%$ reported that they transfer their patients to adult-focused care. When transfer is organized, $75 \%$ of the centers transfer the patients to a formalized Adult Congenital Heart Disease Program. In $67.9 \%$ of these transfers the ACHD program has the same location as pediatric care. The median age of transfer is 18 years for centers with a predetermined age of transfer, and ranges from a median 15 to 18 years of age among centers with has a more flexible policies. Co-morbidities, pregnancy or patient/family request to leave pediatric cardiology were identified as initiators for transfer. Complexity of the heart defect was relatively less important when deciding whether to transfer patients. Only, one-third of the centers that transfer their patients provide a structured preparation for the patient and family. The most frequently reported transition-related behaviors were explaining why and how patients would be transferred and providing education about the heart, heart treatments and health behaviors. A minority of the programs distributed a leaflet or pamphlet or held workshops to facilitate transition. Development of a formal transition program is planned in $78.4 \%$ of the centers that transfer patients.

Conclusion: Although task forces and international guidelines emphasize the importance of a timely transfer and structured transition process of children with congenital heart disease, this is not implemented in all pediatric cardiology programs. This study offers important information to improve healthcare provision for patients with congenital heart disease. Physicians, nurses and administrators working in pediatric cardiology should make their transfer policies explicit and formal transition programs or transition clinics ought to be developed.

\section{Can Adherence to Cardiac Follow-up in Pregnant Women with Congenital Heart Disease Improve by Advanced Practice Nurses? \\ Philip Moons PhD, RN, Kristien Van Deyk, Els Troost, Werner Budts Katholieke Universiteit Leuven, Belgium}

Background: Pregnancy in women with congenital heart disease is associated with cardiac and non-cardiac complications. Cardiac complications occur more frequently in women with congenital heart disease than in the general population, i.c. heart failure; arrhythmias; cardiovascular events; and endocarditis. Hence, cardiac follow-up during pregnancy is imperative for women with congenital heart disease. In 2000, our hospital started with Advanced Practice Nurses in adult congenital heart disease. These Masters-prepared nurses developed and implemented a structured patient education programme, including information on the impact of pregnancy on the heart and the need for trimestrial follow-up visits with a cardiologist during pregnancy. The aims of the present study were (1) to investigate the prevalence of cardiac follow-up during pregnancy in females with congenital heart disease; and (2) to explore whether Advanced Practice Nurses have had an impact on the adherence to cardiac follow-up recommendations.

Methods: This study was a secondary data analysis on data of a descriptive, retrospective study on health behaviour during 
pregnancy, in which 137 women with congenital heart disease were included. Patients born between January 1, 1958 and December 31, 1983, who had been pregnant for at least 20 weeks and who had their last outpatient visit after January 1, 2000, were eligible for inclusion. Patients with isolated patent foramen ovale, rheumatic heart disease, and mental retardation were excluded. For the purpose of this study, we devised a specific questionnaire that could be administered during a telephone interview.

Results: During their first pregnancy, $56.9 \%$ of the patients had follow-up visits with their cardiologist. Before the implementation of Advanced Practice Nursing in our programme, in the year 2000 , the proportion of patients who had cardiac follow-up during pregnancy was $44 \%$. This proportion increased up to $71 \%$ since the Advanced Practice Nursing team started with systematic educational interventions $(\mathrm{Chi} \mathrm{Sq}=9.63 ; \mathrm{P}=0.002)$.

Conclusion: This study shows that a considerable number of women with congenital heart disease do not present for cardiac follow-up during pregnancy. However, we found the possible impact of the Advanced Practice Nursing team in pregestational counselling. Indeed, Advanced Practice Nurses in adult congenital heart disease can contribute to a better understanding of the rationale for cardiac appointments during pregnancy and a better adherence with follow-up recommendations.

Staying Ahead of the Curve: Fostering Productive Change, Innovation, and Collaboration From Within in Pediatric Cardiology

Jeanine Swenson MD, FAAP, FACC, MFT, Janette F. Strasberger, MD Medical College of Wisconsin, University of Wisconsin, Family Therapy Training Institute

Background: While the current "medical care delivery crisis" is ripe for analysis by economists, legislators, policy makers, and other interested parties (NEJM 358:6), the most effective and familycentered, solution-focused, strength-based change can and must come from within the system and the seeds for this change exist currently. Using both a methods and multi-model approach and keeping in mind the somewhat technical focus of modern pediatric cardiology, we hope to rebalance the fine art and science of our great field highlighting twenty-first century strengths such as empathy, emotional intelligence, interpersonal skills, and integration.

Methods: Initially, we will use a diverse and broad-sweeping literature review to analyze possible breakdowns and barriers to practitioner-patient connection, system growth, and engagement by stakeholders. This is important in identifying existing skills and strengths and to visualize a thriving system for the future that includes balance, harmony, leadership and development (Schwartz, et al, IFS model, 1995). Also realizing the unique stresses and challenges of our field, we will also discuss the causes and highlight antidotes for caregiver and professional burn-out. Winter and worsening problems are looming, we may need to identify the branches and blight on our tree of support in order to grow.

Results: With inclusion of our collaborative partners who provide primary and mental health care to patients and families, we can have many entry points into a new educational and caregiving system that is quality-focused, yet inclusive, equitable and sustainable. Internal reform and a slightly different focus in analyzing a multitude of success variables may lead to long-term and lasting results.

Conclusion: Using systemic paradigms and cooperative thinking principles, we hope to provide ways for our audience to stay ahead of the curve in pediatric cardiology, foster change and creativity in their personal workplace, and minimize resistance to promote success for practitioners, patients, families and systems.
Innovation in Patient Care Management Through a Staff Support Role

Lisa Mitchell RN, Heather Meldrum, Kevin McNally, Marie Johnson The Children's Hospital of Philadelphia

Background: In 2007, The Cardiac Center had a large percentage of less experienced staff, both at the bedside and in the charge role. The Charge nurse role also experienced increased responsibilities related to the daily operations of the unit. The leadership group observed an increased need for support to the charge role, as well as support to the newer staff that had completed orientation.

The Clinical Support Role was developed to assist the charge nurse in developing decision making and mentoring skills. The role also added an additional resource for the bedside nurse caring for a patient in a complex clinical environment. Charge nurses in the role needed support to deal with clinical decision making, staffing, and supporting newer staff in patient care.

Methods: The Clinical Support Role was developed using evidence from management literature and conducting focus group meetings with charge nurses regarding their role development needs. Data collection from bedside nurses as part of a retention collaborative was used to identify staff needs from the charge nurse and leadership group. A national database of nursing quality indicators (NDNQI) surveyed staff on management issues and was compared with results from 2006.

The Clinical Support Role includes attendance at morning report to get an overview of patient care needs and to review staffing plan for the day with the charge nurse. This role supports the bedside nurses in various ways including: reviewing the plan of care to assure that all elements of patient care are planned and met, advocacy for bedside nurse participation in interdisciplinary rounds, just in time support and assistance to staff and the charge nurse in procedures and mentoring through unique issues that arise.

Results: The role was implemented in April 2007 and has been continuously evaluated. Strengths of the role include continuous leadership accessibility on the unit. Nurses in the charge nurse role indicated that the hospital wide education provided the basic foundation for the leadership role, however, identified that further development was still needed specific to the Cardiac Center. The Clinical Support Role enabled the charge nurse to have one on one real time support. Charge Nurse meetings were held every other month. Topics included regular evaluation of the staff support role and their development and education. These meetings include time for feedback on the value of the Clinical Support Role.

Data from the most recent NDNQI survey showed a slight increase in perception of management support in 2 of 3 units. Results from the May 2008 Morehead Survey question on how work unit employees feel connected to the supervisor/manager reported a score of 3.76 of 4 .

Conclusion: We need to continue to develop a consistent plan to evaluate and improve the Clinical Support Role. NDNQI results can be dissected to provide more unit and role specific information to evaluate the role. The Clinical Support Role is valued by charge nurses and by staff.

\section{Recombinant Factor Seven Therapy for Postoperative Bleeding in Pediatric Cardiac Surgery Marilyn Torres Pharm. D, D Kimura, A Rossi Miami Children's Hospital}

Background: Postoperative bleeding in cardiac surgery is mutifactorial. Bleeding can result from a definite source at the operation site or an acute coagulopathy. Clinical bleeding may persist despite 
the administration of cryoprecipitate, fresh frozen plasma (FFP), and platelet bleeding.

Methods: Retrospective non-randomized study of forty-nine infants and children with Congenital Heart Disease (CHD), including patients with systemic to pulmonary shunts who received Recombinant Factor Seven (rFVIIa) from January 2005-June 2007 were reviewed for safety and efficacy.

Results: The median age and weight were 0.011 years $(0.003-16)$ and $3.46 \mathrm{~kg}$ (2-65). Before administration of factor VIIa (recombinant), all patients received blood products. Total administered dose $35961.43 \mathrm{mcg}$. The average dose administered of rFVIIa for all patients with (CHD) was $609 \mathrm{mcg}$ $(67-4800 \mathrm{mcg})$. The average dose for the patients with systemic to pulmonary shunts was $76 \mathrm{mcg}$ ( $100 \mathrm{u} 400 \mathrm{mcg}$ ).

The median estimated hourly blood loss from chest tubes one hour before administration of rFVIIa was $77 \mathrm{ml} / \mathrm{hr}$ (range 2-350 ml/hr); one hour after administration was $20 \mathrm{ml} / \mathrm{hr}$ (range $0-175 \mathrm{ml} / \mathrm{hr}$ ); two hours after administration $17 \mathrm{ml} / \mathrm{hr}$ (range $0-42$ ).

Conclusion: Our observations are; seven patients chest was explored after receiving blood products and factor VIIa due to significant bleeding, administration of factor VIIa was associated with a clinical significant decreased in blood loss from chest tubes, (Mann-Whitney test $(p=0.04)$. There was no episode of shunt thrombosis in patients with systemic to pulmonary shunts and no reported adverse effects. Factor VIIa appears to be safe and effective to administer in patients with Congenital Heart Disease.

Enteral Feeding the Neonate with Prostaglandin-Dependent Congenital Heart Disease in the Presence of Umbilical Catheters: Variations in Practice from an International Survey

Erica Wymore MD, E da Cruz, J Kaufman, P Thureen, L Willis University of Colorado Health Sciences Center \& The Children's Hospital Denver

Background: Nutritional strategies for preoperative neonates with prostaglandin (PGE1)-dependent congenital heart disease vary widely among medical caregivers around the world. The presence of umbilical catheters often impacts the decision to enterally feed these PGE1-dependent cardiac infants due to concerns of compromised intestinal perfusion. Albeit, safety of early enteral feeding in the presence of umbilical arterial (UAC) and venous catheters (UVC) has been well-documented in high risk preterm and term infants, no data is available for PGE1-dependent newborns. These partial results of an international multidisciplinary survey specifically evaluate variations in enteral feeding practices in the preoperative neonate with PGE1-dependent congenital heart disease with regards to the presence of umbilical catheters.

Methods: Pediatric practitioners who provide care for neonates with PGE1-dependent congenital heart disease were contacted via email to respond to an internet-based survey using commercially available software (www.surveymonkey.com). The survey encompassed preoperative enteral nutrition strategies and practices for neonates with PGE1-dependent cardiac disease.

Results: The survey had 200 medical provider respondents with a survey completion rate of $90.5 \%$. Of those who completed the survey, $79 \%$ were from the United States (US) and $20.9 \%$ were international respondents. The roles of the responders included: cardiologists $34.8 \%$, neonatologists $37 \%$, pediatric intensivists $6.6 \%$ and $\mathrm{PA} / \mathrm{NP} / \mathrm{RNs} 21.5 \%$. A total of $79 / 143(55 \%)$ US respondents and $27 / 38(71 \%)$ of international respondents reported that it is safe to enterally feed PGE1-dependent neonates with UACs. Comparing cardiologists and neonatalogists, 30/63
(47\%) and 49/67 (71\%) respectively reported it was safe to provide enteral nutrition in the presence of a UAC. Enteral feeding in the presence of a UVC was reportedly safer among all respondents, with 110/143 (76.9\%) US and 33/38 (86.8\%) international medical providers willing to enterally feed such patients. A total of $49 / 63(77.8 \%)$ of cardiologists and $55 / 67(82.1 \%)$ of neonatologists reported the safety of enterally feeding with a UVC in place. Conclusion: This survey demonstrates wide variation in enteral feeding practices in the presence of umbilical catheters for neonates with uncorrected congenital heart disease on PGE1. Notably, providers from abroad and neonatologists appear more comfortable with providing enteral nutrition in the presence of UACs. The comfort of enterally feeding with a UVC in place appears similar among all caregivers. While no guidelines exist regarding preoperative nutrition for this PGE1-dependent population, the results of this survey exhibit the need for prospective studies to determine enteral feeding safety in the presence of umbilical catheters in order to optimize preoperative nutritional delivery.

Addressing the Informational and Developmental Needs of Adolescent Patients with Congenital Heart Disease Kathi Kinnett RN, MSN, CNP, Kathy Gosney, Mark Norris, Phil Khoury, Linda Cripe, Larry Markham, Robert Spicer Cincinnati Children's Hospital Medical Center

Background: Advances in the field of pediatric cardiology have resulted in an increased number of adolescent and young adult survivors of congenital heart disease (CHD). Patients in this age group are known to participate in risk taking behaviors and have an increased incidence of depression. Subspecialists often defer the discussion of these topics to the patients' primary care provider. The objective of this study was to determine whether pediatric cardiologists obtained age appropriate history from adolescent and young adult CHD patients in the outpatient setting.

Methods: From January 2008-August 2008, 75 subjects ages 14-21 years were selected from an urban hospital outpatient cardiology clinic with an ICD code for CHD to participate in an anonymous computer based survey asking subjects to recall how often their cardiologist asked questions regarding physical and mental health, and risk taking behaviors. The severity of disease was classified as mild to moderate (65) and severe (10). Patient responses were categorized as "never/once", "sometimes" and "usually/always".

Results: "Usually/always" was reported in response to questions regarding physical health: medications 65\% (48/74), cardiac symptoms $90 \%$ (67/75); dental care 30\% (23/75), diet 50\% (37/ $74)$, exercise $60 \%$ (50/75); mental health: depression $7 \%$ (6/75); risk taking behaviors: sexual activity $10 \%(8 / 75)$, STI prevention $6 \%(5 / 75)$, tattooing $7 \%(5 / 75)$, body piercing $5 \%(5 / 75)$, drug use $13 \%$ (10/75), alcohol use 12\% (9/75), smoking $20 \%$ (15/75). Conclusion: When taking a history in an adolescent or young adult with CHD, pediatric cardiologists frequently ask questions regarding physical health. Rarely, however, do they inquire about mental health or risk taking behaviors. As this patient population does not regularly see their primary care provider, the cardiologist is left as the only individual asking these important health related questions. Risk taking behaviors and neglect of age appropriate health maintenance can both lead to devastating results for all adolescents, particularly those with CHD. Consequences of risk taking behaviors may directly impact short and long term cardiovascular health. The importance and implications of age appropriate history taking must be emphasized in the pediatric cardiology setting. 
AIIM for Best Practice

Kirsten Schroeder RN, Jennifer Bishop, Megan Crane

British Columbia Children's Hospital

Background: Creating a culture of safety is a necessity in every work place. At British Columbia Children's Hospital (BCCH) with the guidance of the Canadian Patient Safety Institution and the Canadian Nurses Association, we promote a safe environment for our patients and health care professionals. Current initiatives at $\mathrm{BCCH}$ include daily safety rounds, an "AIIM for best practice" statement, and a new reporting system for near misses and adverse events.

Methods: Analysis of current safety practices and respective plans for improving safety were made by front-line leadership and staff of a pediatric cardiac and medical unit. Areas for improvement were found in attendance and effectiveness of safety rounds and performance of standard safety checks. Daily morning safety rounds in place of bi-weekly afternoon safety rounds and a mnemonic abbreviation "AIIM for best practice" were developed and implemented following a more condensed shift handover. AIIM stands for ABC, IV, Identification (ID) bands and Medications. By following the AIIM statement, nurses would first check each patient's $\mathrm{ABCs}$, ensure safety equipment is present and functioning, perform an IV site to source assessment, and verify the ID band and allergy status of each of their patients prior to dispensing medications and completing a full head to toe assessment.

The aforementioned initiatives corresponded well with the British Columbia (BC) wide Patient Safety and Learning System (PSLS) for reporting and analyzing safety concerns. PSLS replaces existing paper-based incident reports with an electronic reporting system that allows a collective approach to sharing information, promoting a culture of safety for clients and health care professionals across BC. It also allows for anonymous reporting improving system-wide safety and trust.

Results: The combined implementation of daily safety rounds, the "AIIM for best practice" statement, and the PSLS initiatives marked a measurable increase in safe practices. Trends such as a decreased number and severity of interstitial IV's, an increased number of patients wearing ID bands, and appropriate safety equipment in rooms were observed. Positive feedback from nurses and improved attendance also revealed that daily safety rounds were more convenient when held in the morning. The PSLS also proved to be a useful tool in tracking the number of incident reports such as interstitial IV's.

Conclusion: Evidence-based practice in conjunction with a culture of curiosity and learning at $\mathrm{BCCH}$ demonstrates that we prioritize safety and are continually striving for best practice and optimal client well-being. The Code of Ethics for Registered Nurses of British Columbia states we provide "safe, competent and ethical care", which we are achieving through these initiatives. Daily safety rounds, the "AIIM for best practice" statement and PSLS are a few steps toward optimizing patient and staff safety. Teamwork and the opportunity to share safety events collaboratively have created a no-blame environment where health care professionals are able to learn together and provide safe patient care.

Acute Kidney Injury (AKI) Associated with Angiotensin Converting Enzyme (ACE) Inhibitor Initiation after Pediatric Cardiothoracic Surgery

Brady Moffett PharmD, Michelle Adusei, Julia Kuzin, Princy Mohan, Stuart L. Goldstein, Antonio R. Mott

Texas Children's Hospital, Baylor College of Medicine

Background: Angiotensin converting enzyme (ACE) inhibitors are used in pediatric patients (pts) who have had cardiac surgery.
Acute kidney injury (AKI) can occur after initiation of ACE inhibitors therapy. The primary objective of this study is to determine risk factors for the development of AKI after initiation of ACE inhibitor therapy in this group of patients.

Methods: Using the pharmacy and surgical databases, we identified all pts $<18$ yo who: (1) had cardiac surgery and (2) received an ACE inhibitor after a cardiac surgery from January 2006 to December 2007. Pts who did not have a baseline serum creatinine (SCr) or at least one SCr obtained after ACE inhibitor initiation were excluded. Data collected included: demographic information and cardiac pathophysiology/surgery. Also collected were ACE inhibitor type, dose, frequency, and therapy initiation date. Baseline, daily, and maximum SCr values were also collected. Serum creatinine clearance $(\mathrm{CrCl})$ was calculated using the modified Schwarz equation. The use of diuretic and/or other nephrotoxic medications was documented. AKI was defined as a change in pRIFLE criteria within 48 hours of initiation or after increase in ACE inhibitor dose.

Descriptive statistics were used for initial evaluation of the cohort. Ordinal multivariate logistic regression analysis with clustering of standard errors was used to determine significant factors for development of ACE induced AKI. A p value of $<0.05$ was set a priori.

Results: There were 409 pts that comprised 448 admissions [Neonate $(\mathrm{n}=76,17 \%)$, Infants $(\mathrm{n}=211,47 \%)$, Children $(\mathrm{n}=139,31 \%)$, and Adolescents $(\mathrm{n}=22,5 \%)]$. Cyanosis was present in $157 / 448(35 \%)$ pts. Most common cardiac operations were: Bidirectional Glenn shunt $(n=63)$, Fontan $(n=62)$, VSD closure $(\mathrm{n}=42)$, Norwood $(\mathrm{n}=34)$, and CAVC repair $(\mathrm{n}=29)$. The ACE of choice was Enalapril in $264(59 \%)$ pts and captopril in $184(41 \%)$. AKI occurred in $166(37 \%)$ pts $(\mathrm{R}=23 \%, \mathrm{I}=4 \%$, $\mathrm{F}=10 \%)$. ACE inhibitor induced AKI occurred in $99(22 \%)$ pts $(\mathrm{R}=14 \%, \mathrm{I}=3 \%, \mathrm{~F}=5 \%)$. Multivariate regression analysis identified young age (OR 0.98, $\mathrm{p}<0.05$ ), low baseline $\mathrm{CrCl}(\mathrm{OR}$ $1.01, \mathrm{p}<0.05)$, cyanosis (OR 1.7, p $<0.05$ ), and furosemide use (OR 3.6, $\mathrm{p}<0.05)$ as risk factors. Pts with ACE inhibitor induced AKI received a higher dose of furosemide (1.7 vs $2.2 \mathrm{mg} / \mathrm{kg} /$ day, $\mathrm{p}<0.05)$.

Conclusion: ACE inhibition therapy is commonly used in pediatric pts who had cardiac surgery. ACE inhibitor induced AKI occurs in these patients. Preliminary data suggest that risk factors for developing ACE inhibitor induced AKI include young age, low baseline $\mathrm{CrCl}$, cyanosis, and high dose furosemide.

\section{Short and Medium Term Outcomes for Patients with Modified Extra-Cardiac Fontan \\ Daniel Rowland MD, Tracey John RN, Roberta Rodeman RN, Bethany Hashiguchi, Mark Galantowicz MD \\ Nationwide Children's Hospital, The Obio State University School of Medicine and Public Health}

Background: An ideal Fontan connection should maximize flow dynamics while minimizing complications such as arrhythmias, myocardial dysfunction and thrombosis. The original Fontan operation has undergone numerous modifications since its initial description in 1968 in an attempt to meet these goals. In the current era, the intra-cardiac lateral tunnel (ICLT) and the extracardiac tube graft (ECTG) are the two primary surgical techniques utilized. Each technique offers both advantages and disadvantages. The ICLT poses the risk of arrhythmias by placing extensive suture lines in the right atrium and requires cardiac arrest. While the ECTG avoids atrial suture lines and cardiac arrest, it does not allow growth. A modified extra-cardiac Fontan (MECF) which 
creates a Fontan pathway utilizing the outer surface of the right atrium, native pericardium and a donor pericardial roof offers a third approach. Advantages of this modification include the avoidance of atrial suture lines, does not require arresting the heart, has the ability to grow, and a theoretical possibility of pulsatile blood flow through the Fontan circuit from the heart wall motion and respiratory cycle.

Methods: An IRB approved retrospective chart review was preformed of all patients who underwent a Fontan procedure between $11 / 1 / 2001$ to $8 / 11 / 2008$ at Nationwide Children's Hospital. Pre-operative data collected included patient demographics, ventricular morphology, atrioventricular valve regurgitation (AVVR), presence of sinus rhythm (NSR), pre-existing arrhythmias and ventricular function (VFx). Intra-operative data included bypass time (CPB), and inotrope score (ORIS). Postoperative data collected included ICU LOS, hospital LOS, chest tube days (CTd), days until feeding, time to extubation, postoperative complications and 24 hour inotrope score(24IS). Long term follow-up focused on AVVR, maintenance of NSR, new arrhythmias, thrombosis and VFx.

Results: A total of 100 patients were identified with 14 pts excluded due to redo Fontan or other complex issues. The remaining 86 patients were divided into 3 groups based on surgical technique; 40 MECF pts, 28 ICLT pts and 18 ECTG pts. There were 4 early ( 3 ICLT, 1 ECTG) post-operative deaths and 2 late deaths (1 ECTG, $1 \mathrm{MECF}$ ). One MECF required conversion to ECTG due to poor hemodyanmcs. There was no significant difference in any pre-operative variables between groups. MECF were least likely to be fenestrated $(20 \%)$, had no XCT, shortest CPT and the lowest ORIS. The mean time to extubation was similar and less than 4 hours for all groups. ICU LOS and hospital LOS were similar; however, MECF did require longer CTd compared to both ICLT and ECTG. The mean follow-up was 17, 35, and 49 months for MECF, ICLT and ECTC respectively. 81\% of all pts were in NSR. No significant intergroup difference in arrhythmias, NSR or thrombus was found, although there was a trend for preserved NSR in MECF (90\%) and ECTG (80\%) vs ICLT $(66 \%)$.

Conclusion: The MECF minimizes CPB, post-op inotropes, and eliminates XCT while preserving NSR. Longer post-op effusions are associated with this technique. The incidence of short and medium term arrhythmias and thrombosis are low. Longer detailed follow-up is needed to determine if long term benefits exist.

\section{Transition: What do Pediatric Cardiologists Understand About it Anyway? \\ Desire Fleck PhD(c), CRNP, Barbara Riegel, DNSc, RN \\ The Children's Hospital of Philadelphia/The University of Pennsylvania School of Medicine}

Background: One in ten neonates is affected by congenital heart disease (CHD). Adults with CHD often see pediatric cardiologists, even though transition to self-care and an adult provider is desirable. Transition is a multifaceted process where adolescents and young adults prepare to assume responsibility for self-care. Although pediatric providers' attitudes and beliefs are thought to influence successful transition, there are limited empirical data to support this assumption. The purpose of this preliminary descriptive qualitative study was to explore pediatric cardiologists' understanding of and participation in transition of CHD patients. Methods: A purposive sample of four pediatric cardiologists was interviewed about their understanding of and participation in transition of CHD patients. Data were collected using in-depth semi-structured interviews guided by an interview guide. Interviews were audiotaped, transcribed verbatim, and analyzed using content analysis. Content codes were systematically organized into conceptual categories. Categories were collapsed for parsimony.

Results: The CHD experience of the four pediatric cardiologists ranged from 13-20 years with 2-20 years at a large urban academic medical center. All providers viewed transition as important and 3 out of 4 reported transferring care of patients to the adult center. Providers reported participating in transition by introducing the topic, sending a letter to the new center, and assessing the patient's/family's response to the suggestion of transition to adult care. Nonetheless, 3 of 4 pediatric cardiologists expressed a lack of understanding about the subtleties of the transition process. During content analysis, 190 codes were identified and reduced to 14 categories clustered into four themes: 1) factors that promote transition; 2) barriers to transition; 3) the process of transition; and 4) successful transition. Factors that promote transition included the presence of an internationally known expert in adult CHD care and an eagerness and willingness to learn and participate in the process. Barriers included time constraints that prohibit conversations about transition, lack of knowledge about or how to transition patients, and concern regarding accessibility to adult care and insurance. All participants identified the need for improvement in transitioning adult patients with CHD

Conclusion: While pediatric cardiologists understand the mechanics of transitioning adult CHD patients to adult care, they had little understanding of the full depth and breadth of what is required for a successful transition. Further research aimed at understanding the CHD patient's perspective on transition may provide insights to improve the care provided to adults with CHD.

Continuous Renal Oximetry Correlates with Renal Dysfunction, Systemic Oxygen Delivery, and Post-Operative Course in Neonates and Infants with Congenital Heart Disease Undergoing Biventricular Repair

Gabe Owens M.D., PhD., Lindsay Ryerson, Christopher Jackson, John Charpie

University of Michigan Congenital Heart Center

Background: With advances in surgical techniques and perioperative care, mortality has declined significantly in infants with congenital heart disease undergoing cardiac repair or palliation. As a result, there has been a greater emphasis placed on limiting morbidity following cardiac operations in children. Acute renal injury is a frequent complication following cardiopulmonary bypass surgery that has been correlated with poor outcomes, prolonged hospital stays, and increased mortality. Standard methods for evaluating renal function include urine output, serum creatinine, and more recently changes in urinary biomarkers. None of these measures are particularly sensitive or early indicators of renal dysfunction that allow intervention in real-time. Near infrared spectroscopy (NIRS) is a newer noninvasive technology that evaluates regional oximetry and has been found to be a surrogate measure of regional blood flow and organ perfusion. The objective of this study was to evaluate if continuous renal oximetry correlates with renal injury and adverse outcomes following cardiac surgery in neonates and infants.

Methods: Neonates and infants (0-12 months of age) without preexisting renal dysfunction who were undergoing biventricular repair with $\mathrm{CPB}$ were enrolled in this prospective observational study. All patients had an INVOS Pediatric near-infrared spectroscopy sensor (Somanetics Corp., Troy Michigan) placed above the 
right kidney, and continuous regional oximetry data were collected for the first 48 post-operative hours. Oximetry data were correlated with post-operative course, hemodynamics, standard laboratory data, and the occurrence of renal injury.

Results: To date, 27 subjects have been enrolled in the study. In the immediate post-operative period, 13 of 27 subjects (48\%) had elevated creatinine levels from preoperative values. Only four of these subjects progressed to meet Pediatric RIFLE criteria for renal dysfunction for the subsequent 48 hours by both serum creatinine and urine output criteria. These 4 subjects had prolonged (10 hrs) and persistent renal oximetry measurements $>$; 2-SD below their own baseline values compared to subjects without renal dysfunction $(1.7 \mathrm{hrs}, \mathrm{p}<0.001)$. Furthermore, a cohort of subjects $(\mathrm{n}=11)$ who had greater than $2 \mathrm{hrs}$ of renal oximetry values $>$; 2-SD below baseline had significantly higher creatinine levels $(0.8$ vs. $0.4, \mathrm{p}=0.004)$, lactates $(4.3$ vs. $2.4, \mathrm{p}=0.02)$, and lowest $\mathrm{pH}(7.28$ vs. $7.32, \mathrm{p}=0.04)$. These patients also had significantly longer mechanical ventilation days ( 7 vs. $3, p=0.003)$, ICU (11 vs. $4, \mathrm{p}=0.003)$ and overall lengths of stay (16 vs. 8.5, $\mathrm{p}=0.005)$. There were no significant differences in cardiopulmonary bypass times, aortic cross clamp times, circulatory arrest times, or inotropic scores between subjects with and without renal dysfunction. No subject required dialysis.

Conclusion: Prolonged low renal near-infrared oximetry appears to correlate with renal dysfunction, decreased systemic oxygen delivery, and overall post-operative course in neonates and infants with congenital heart disease undergoing biventricular repair. Near-infrared spectroscopy may play an important role in helping identify renal injury in a real-time, noninvasive manner, and may help guide interventions aimed at preserving post-operative renal function and decreasing overall morbidity and mortality.

Risk Factors for Refractory Hypotension After Cardiopulmonary Bypass in Neonates

Lois Chiu MD, David Bateman MD, Veniamin Ratner MD, Ganga Krishnamurthy $M D$

Morgan Stanley Children's Hospital of New York, Columbia University

Background: Systemic inflammatory response syndrome occurs in up to $20-25 \%$ of neonates who undergo cardiac surgery under cardiopulmonary bypass (CPB). Clinical manifestations include a hyperdynamic circulatory state with refractory hypotension $(\mathrm{RH})$ and capillary leak that is both fluid and pressor resistant. $\mathrm{RH}$ in this context poses a management challenge and is an important cause of postoperative morbidity and mortality. Risk factors that could potentially predispose certain infants to develop refractory hypotension in the postoperative setting remain unknown.

The objective of this study was to identify potential risk factors for the development of refractory hypotension in neonates after palliative or reparative cardiac surgery performed under $\mathrm{CPB}$.

Methods: A retrospective chart review was conducted of all neonates who underwent either palliative or reparative surgery under $\mathrm{CPB}$ between January 2006 and September 2007. The following variables were examined as potential risk factors for RH: Birth weight, gender, age at surgery, preoperative need for mechanical ventilation, type of congenital heart defect, surgical risk category based on a consensus conference (Jenkins, 2002), intraoperative use of etomidate as an induction agent, lack of intraoperative corticosteroids, total $\mathrm{CPB}$ time, aortic cross clamp time and circulatory arrest time. Univariate and multivariate logistic regression methods were used to determine statistical significance ( $\mathrm{p}<0.05$ was considered significant).

Results: Of a total of 184 neonates who underwent surgery under CPB, 44 (24\%) developed RH. Using Pearson chi-square test, the use of etomidate as an induction agent, and lack of administration of intraoperative corticosteroids were factors that significantly increased the risk for development of $\mathrm{RH}$ in the postoperative period $(\mathrm{p}<0.01)$. Similarly, under multivariate logistic regression, the use of etomidate and lack of administration of corticosteroids intraoperatively were found to increase the risk of $\mathrm{RH}$ by odds ratios of $4.38(95 \% \mathrm{CI} 1.27-15.10 ; \mathrm{p}=0.02)$ and 2.28 (95\% CI 1.09-4.75; p $=0.03$ ) respectively.

Conclusion: In this retrospective study, the use of etomidate as an anesthetic induction agent and the non-administration of intraoperative corticosteroids emerge as significant risk factors for the development of refractory hypotension after congenital heart surgery in the neonatal period. Etomidate, a hypnotic, carboxylated imidazole derivative is used as an induction agent in rapid sequence intubation. It is speculated that etomidate with its previously well described suppressive effect on glucocorticoid synthesis induces a state of "relative adrenal insufficiency" leading to a picture of vasopressor resistant hypotension.

\section{Hemodynamic Consequence of Interventional Cardiac Catheterization in the Early Postoperative Period after Congenital Heart Surgery Eric Eason DO, Khan DM, Ross AF, Suciu GP Congenital Heart Institute at Miami Children's Hospital}

Background: While still considered to be high risk procedures, interventional and diagnostic cardiac catheterization in the early postoperative period are being performed more frequently in the current era. There is no data currently available concerning the acute hemodynamic consequences of these procedures.

Methods: We completed a retrospective review of all catheterizations performed between 2/2002 and 2/2008 that occurred within 30 days of $\mathrm{CHS}$. Procedures were performed as a result of failure to progress or hemodynamic deterioration in the early postoperative period. The physiologic consequence of these procedures was determined by assessing the affects pre and post procedure on hemodynamic parameters, metabolic markers of tissue perfusion and renal function, and inotrope requirements.

Results: During the study period, there were 1799 congenital heart surgeries (1545 cardiopulmonary bypass cases). There were 100 patients $(6 \%)$ at a median age of $124 \mathrm{~d}(5 \mathrm{~d}-16 \mathrm{yr}$; neonates $\mathrm{n}=38$, infants $\mathrm{n}=35)$ and mean weight $7.73 \mathrm{~kg}(1.7-37.8 \mathrm{~kg})$ who underwent catheterization on mean postoperative d $11(0-30$ d). Sixty-three cases received a total of 71 interventions. Prior to catheterization, $62 \%$ were intubated and $11 \%$ were requiring mechanical cardiopulmonary support (CPS). Complications in the interventional group included arrhythmias in 5, intimal tears with insignificant hemorrhagic loss in 2, atrial septal stent failure requiring surgical retrieval in 1 , and cardiac arrest in 1 patient. Consequence of acute postoperative intervention included an increase in creatinine from 0.6 pre cath to 0.84 immediate post cath $(\mathrm{p}=0.03)$ and back to 0.7 at $24 \mathrm{hrs}(\mathrm{p}=0.04)$. Blood lactate decreased from 1.84 pre-cath to 1.27 at 24 hrs post cath $(\mathrm{p}=0.01)$ while no significant change was seen in the diagnostic group $(\mathrm{p}=0.7)$. There was no significant change in HR, systolic or diastolic $\mathrm{BP}, \mathrm{sPO}_{2}$, or inotrope scores immediately or at $24 \mathrm{hrs}$ post cath. There were 5 patients who required pre-catheterization CPS with 2 being weaned off support after cath. One patient required CPS immediately post cath. Seven patients undergoing intervention died at 0-12 days post cath (mean 5d). Survival for patients not requiring cardiac catheterization postoperatively was 99\%. Survival for patients undergoing diagnostic cath $(81 \%)$ did not differ significantly for those who underwent intervention $(90 \%)$. 
Conclusion: Cardiac catheterization was performed on $5.7 \%$ of patients undergoing CHS in our series. Acute postoperative cardiac catheter intervention was performed in $3.5 \%$. Intervention or diagnostic catheterization was performed on those patients at highest risk for death in the postoperative period. Catheter intervention did not increase the risk for death. Those patients undergoing catheter intervention did not seem to undergo any significant negative hemodynamic or renal consequence from their catheterization but achieved mild improvement in tissue perfusion.

Assessment of Inotrope Score as a Predictor of Morbidity and Mortality in Infants after Cardiopulmonary Bypass

Michael Gaies MD, MPH, Yen AH, Napoli M, Gurney JG, Gajarski RJ, Charpie JR, Hirsch JC

University of Michigan

Background: Since being initially described by Wernovsky et al. (Circulation 1995;92(8):2226-35), inotrope score has been used by several investigators as a measure of illness severity in patients undergoing congenital heart surgery (CHS). However, inotrope score has not been validated as an independent predictor of outcome after CHS.

Methods: Statistical properties of a revised inotrope score were evaluated for predicting poor outcome following CHS with cardiopulmonary bypass. From a consecutive case series of 200 infants, ages 6 months or younger and treated between August 2007 and June 2008 at our institution, $173(86.5 \%)$ had complete hourly inotrope doses recorded for the first 48 hours after surgery from medical records and were included in this retrospective analysis. The inotrope score (IS) was calculated as described by Wernovsky et al: dopamine dose $(\mathrm{mcg} / \mathrm{kg} / \mathrm{min})+$ dobutamine $(\mathrm{mcg} / \mathrm{kg} / \mathrm{min})+100 \times$ epinephrine $(\mathrm{mcg} / \mathrm{kg} / \mathrm{min})$. We expanded the IS to define a vasoactive/inotrope score (VIS) represented by IS $+10 \mathrm{x}$ milrinone $(\mathrm{mcg} / \mathrm{kg} / \mathrm{min})+10,000 \mathrm{x}$ vasopressin (units/ $\mathrm{kg} / \mathrm{min}$ ). Mean IS and VIS for the first and subsequent 24 hours were calculated by adding hourly scores and dividing by 24 during each time period. Areas under the receiver operating characteristic (ROC) curves were compared based on cutpoints using a priori combinations of 24 and 48 hour maximum VIS versus maximum IS, and mean VIS versus mean IS. A dichotomous composite morbidity and mortality outcome variable (poor outcome) was reached if any of the following occurred: hospital or 30-day mortality, cardiac arrest, mechanical circulatory support, dialysis, or central nervous system injury.

Results: A poor outcome was experienced by 35 (20\%) patients, including 20 deaths. The ROC areas comparing each of the four inotropic score comparison groups were not statistically different from one another $(\mathrm{p}=0.63)$, but seemed to favor the VIS maximum $(0.829 \pm 0.035$ standard error) over the IS maximum $(0.78 \pm 0.042)$, and the VIS mean $(0.823 \pm 0.029)$ over the IS mean $(0.766 \pm 0.046)$. The best performing a priori cutoff point was for the maximum VIS, in which patients had a maximum IS $>20$ during the first 24 hours or $>15$ during the second 24 hours. This cutoff produced a sensitivity of 0.714 and a specificity of 0.797 . Multivariable logistic regression (controlling for diagnosis using the RACHS-1 system) was used to estimate the relative odds of having a poor outcome for each of the five cutoff points considered. Again the results favored the maximum VIS (adjusted OR $=8.08,95 \%$ CI 3.40-19.2, $\mathrm{p}<0.001$ ) at the cutoff point described above over the IS method and other cutoffs.

Conclusion: In a series of infants undergoing CHS at a single institution, post-operative inotropic support appears to be a strong independent predictor of poor outcome. Our results suggest that

the VIS measure of inotropic support provides improved predictive benefit over the IS, and may be best expressed as a maximum score rather than a mean. Prospective validation of this measure in a larger study is necessary to confirm its utility as an early marker of illness severity after CHS.

\section{Association Between RACHS-1 Classification and Long-Term Survival \\ Signe Holm Larsen MD, Soren Paaske Johnsen, Kristian Emmertsen, Vibeke Hjortdal \\ Aarbus University Hospital Skejby, Denmark}

Background: The Risk Adjusted Classification for Congenital Heart Surgery (RACHS-1) was created in order to compare in-hospital survival for patients undergoing congenital heart surgery. We have previously shown that this classification predicted in-hospital survival in our patient series, however it has remained uncertain to which extent the RACHS-1 classification could also predict longterm survival.

Methods: We identified 1039 operations performed in 878 children from 1996 to 2002 at Aarhus University Hospital, Skejby (72\% were operated once, $20 \%$ twice, $8 \%$ three times and $0.4 \%$ four times). All patients were followed-up from date of surgery until date of death, emigration or end of follow-up (August 8th 2008), whichever came first, using the Danish National Patient Register. Each operation was matched with a RACHS-1 risk category. For patients operated multiple times only the first operation was included. The ability of the RACHS-1 classification to predict long-term survival was examined by estimating the area under the ROC curve. Cox regression analysis was used to examine if sex, age and weight in addition to the RACHS-1 classification were associated with long-term survival.

Results: RACHS-1 could be applied in 93\% of the operations. Mean length of follow-up was 8.1 years, giving a total follow-up at 6603 years. Thirty days survival was 94\% (95\% confidence interval 92-95\%) and overall survival was $85 \%$ (81-88\%) after up to 12.6 years of follow-up. Overall survival in RACHS-1 category one was 90\% (65-98\%); category two 94\% (91-96\%); category three $78 \%(72-82 \%)$ and category four $65 \%(52-75 \%)$. The area under the ROC curve was 0.78 (95\% confidence interval $0.74-0.83$ ). There was a significant difference in long-term survival between the RACHS-1 categories, when comparing category two, three and four to one $(\mathrm{p}<0.001)$, but not when comparing category two and one $(\mathrm{p}=0.21)$. Besides the RACHS1 classification weight, but not age and sex, was associated with long-term survival.

Conclusion: The RACHS-1 classification seems to be associated to long-term survival as well. However, we were not able to show a difference between RACHS-1 category one and two. Further studies are needed to clarify the predictive value of the RACHS-1 classification for long-term survival.

\author{
An Innovative Way to Revalidate Cardiac Nursing \\ Competency: An Annual Staff Skills Fair \\ Karen Goldschmidt MSN, RNC, Jeong-Sook Rim BA, RN, CPN; \\ Marilyn Blaustein BSN, RN; Donna Rust, MSN, RN, CRNP; \\ Linda Goss MSN, RN \\ The Children's Hospital of Philadelphia
}

Background: The Cardiac Center at The Children's Hospital of Philadelphia is comprised of three separate units: the Cardiac Intensive Care Unit, The Cardiac Care Unit and The Cardiac 
Preparation Recovery Unit with over 250 staff members. Revalidating skills on an annual basis, as required by the Joint Commission, can be a challenge for a large center providing specialty services to a wide variety of patients.

Methods: A skills fair committee was developed that included staff nurses, the clinical nurse specialist, the education nurse specialist and the nurse manager. Skills requiring revalidation were identified by analysis of: incident reports, Joint Commission Patient safety goals, institutional initiatives, and anecdotal data collected from patient rounds by the clinical nurse specialist. Critical high risk, low volume skills were also included. Eleven skill stations were identified through prioritizing patient care needs and matching nursing skills sets across all areas. Staff were identified to coordinate skills stations. Station coordinators met with designated clinical experts and designed the station with assistance of the nurse educator. Coordinators were responsible for training additional staff to be qualified observers for each station. The skills fair committee identified all clinician roles throughout the center and defined which skills each clinician was required to complete. The skills fair was offered over a 5 day period over a variety of shifts in an effort to accommodate patient care needs and individual nurses' schedules. Flexibility in scheduling was maintained so that nurses could attend the skills fair at a time convenient to their schedule. An online evaluation tool was created for staff to complete upon finishing the skills fair.

Results: Two hundred and thirty five nurses completed the skills fair. The skills fair was open for a period of 60 hours and was staffed by 59 qualified observers. Survey results revealed that the majority of staff worked 4 hours of additional time to complete the stations. Qualified observers were provided with 8 hours of paid time within their status to teach and were asked to work a minimum of 12 hours.

Conclusion: This is the fifth year that the Cardiac Center has run the skills fair. The skills fair is an effective way to revalidate annual competency. The skills fair builds teamwork and staff morale in the unit. Staff enjoy the atmosphere of sharing their expertise and learning from one another. To encourage completion, prize drawings were held at the end of each day and all nurses who completed the skills fair were given a Cardiac Center t-shirt commemorating the event. Each year we work at streamlining our process. This year improvements were seen in online evaluation, reduction in amount of stations taught and increased time paid to qualified observers to teach. Having paid time allowed for less qualified observers and increased the quality of teaching. The majority of the stations and teachers were rated in evaluations as "very good" to "excellent". The next step is to conduct a Strengths, Weaknesses, Opportunities \& Threat (SWOT) analysis of each station to determine areas for further improvement.

Comparison of Shunt Type at Norwood Palliation with Clinical Assessment and Outcome through Fontan Completion Eric Grabam MD, Jacob W. Phillips, Girish S. Shirali, Scott M. Bradley, Varsha M. Bandisode, Andrew M. Atz Medical University of South Carolina

Background: A modification to the Norwood procedure involving a right ventricle to pulmonary artery (RV-PA) shunt may improve early postoperative outcomes. Concerns remain about the effect of the RV-PA shunt and the accompanying ventriculotomy on ventricular and tricuspid valve function, pulmonary artery growth, candidacy for and outcome following Fontan completion.

Methods: We compared 76 patients who underwent the Norwood procedure, from January 2000 through April 2005, with a modified Blalock-Taussig shunt $(\mathrm{mBT}, \mathrm{n}=35)$ versus RV-PA shunt $(n=41)$. Patients were followed until Fontan palliation or October 1, 2008. Courses were reviewed and compared between groups. Values are median (range), or mean $\pm \mathrm{SD}$ as appropriate. Results: Mortality occurred in 6/35 mBT and 5/41 RV-PA patients while hospitalized for the initial Norwood palliation, $\mathrm{p}=0.75$. Interstage deaths occurred in 6 additional patients with a mBT shunt and 2 patients with an RV-PA shunt, $\mathrm{p}=0.13$. There was one hospital death at second stage palliation in the RV-PA shunt group, $\mathrm{p}=1.0$. No patient in either group expired between stage two palliation and Fontan completion. There were no significant hemodynamic differences between groups at the pre-Fontan cardiac catheterization. The Nakata index was $272 \pm 57 \mathrm{~mm}^{2} / \mathrm{m}^{2}$ and $275 \pm 72 \mathrm{~mm}^{2} / \mathrm{m}^{2}$ in the $\mathrm{mBT}$ and RV-PA shunt groups respectively, $\mathrm{p}=0.9$. Pre-Fontan echocardiographic assessment of ventricular dysfunction, graded 1(none)-4(severe), revealed worse ventricular function in patients who had received an RV-PA shunt; $1(1-2) \mathrm{mBT}$ versus $2(1-4) \mathrm{RV}-\mathrm{PA}, \mathrm{p}=0.01$. The severity of tricuspid regurgitation was similar in both groups. There were no differences between groups at the Fontan hospitalization for mortality, time on mechanical ventilation, intensive care stay or total hospital stay. A single patient in each group required a cardiac transplant prior to Fontan completion. Fontan completion has been delayed, while considering cardiac transplantation, in 1 patient in the mBT group and 8 patients in the RV-PA group, $\mathrm{p}=0.07$

Conclusion: The RV-PA shunt is associated with increased echocardiographic evidence of RV dysfunction at the time of pre-Fontan assessment. Despite similar hemodynamic data between groups, there is a trend for delaying Fontan completion in the RV-PA group. In patients who underwent Fontan completion, post-operative outcomes were not different between groups. Whether the delay in Fontan completion in the RV-PA group is justified warrants further evaluation.

\section{Heterotaxy Syndrome: Institutional Surveillance for and Management of Intestinal Malrotation \\ Hayden Zaccagni MD, Andy Atz MD, Tim McQuinn MD Medical University of South Carolina}

Background: Heterotaxy syndrome (HS) complicates the perioperative course of infants born with congenital heart disease, especially those with functional single ventricle (SV) lesions. It remains an independent risk factor for morbidity following palliative or corrective cardiac surgeries. Screening and management of the extracardiac manifestations of HS is infrequently described in this population. The aim of this report is to examine our institutional management of HS patients with respect to intestinal malrotation in a cohort of patients with SV physiology.

Methods: During the period from August 2002 to May 2007, we prospectively screened all neonates with SV physiology for extracardiac abnormalities. Patients with echocardiographic or radiographic suspicion for HS were evaluated with abdominal ultrasound. If the ultrasound was supportive of a diagnosis of HS, an upper GI with small bowel follow through (SBFT) was performed to screen for malrotation. If malrotation was identified, the patient underwent a Ladd's procedure after the initial NHS recovery and prior to discharge from the hospital. Chart review of these patients was performed looking for total hospital length of stay (LOS), pediatric cardiac intensive care unit (PCICU) LOS after neonatal heart surgery (NHS), PCICU LOS after Ladd's procedure, time between NHS and Ladd's procedure, and complications after Ladd's procedure. 
Results: 112 functional SV patients required cardiac surgery in first 30 days of life from 2002 to 2007. 17 were identified to have HS. 2 of the 17 patients suffered abdominal catastrophes due to malrotation and so were not considered candidates for NHS. 10 of the 17 were found to have asymptomatic malrotation and were taken to the OR for prophylactic Ladd's procedure. 5 of the $17 \mathrm{did}$ not have malrotation. When comparing the latter two groups, median total hospital LOS was 35 days for both groups, median PCICU LOS after NHS was 13 days for the group with malrotation and 24 days for the group without malrotation, and $90 \%$ in the malrotation group went on to Glenn compared to $40 \%$ in the non-malrotation group. For the malrotation group, median PCICU LOS after Ladd's procedure was 1.5 days, and the median time between NHS and Ladd's procedure was 20 days. 1 patient had peritonitis with dehiscence after her Ladd's procedure with gastrostomy tube placement, but was able to be discharged home after full recovery.

Conclusion: Data documenting the incidence of midgut volvulus and GI complications in HS patients with malrotation are scanty. In our cohort, 12 of 17 with HS demonstrated malrotation, and 2/ 12 with malrotation died prior to any palliative cardiac procedure as a direct consequence of their malrotation. Of the 10 patients with palliated hearts that underwent Ladd's procedure, there was one major complication, and no deaths were attributable to GI intervention. Our data suggest that Ladd's procedure adds little to the risk or duration of neonatal hospitalization and supports consideration of aggressive identification and management of this potentially lethal malformation in HS.

The Creation and Use of a Pediatric Cardiac Catheterization Database to Improve Efficiency of Patient Flow

Heather Shockley RN, BSN, CCRN, C Denise Kaufholz CPNP, RN, MSN The Children's Hospital, Denver

Background: The scheduling of patients through a pediatric catheterization lab is subjective. The unpredictability of case length due to subjective scheduling may cause unnecessary delay of cases, overtime shifts for staff, inpatient admissions that would not otherwise be warranted, and at times, case cancellations. The issues related to subjective scheduling of patients directly impacts patients, their families, and staff satisfaction.

After extensive review, there was not sufficient data pertaining to objective scheduling tools to assist in forecasting the length of case for the pediatric cardiac catheterization patient. Therefore, a database was created to pursue the creation of an objective scheduling tool for the pediatric cardiac catheterization lab. Results from the database will be evaluated to implement changes in the scheduling process to ensure proper scheduling times which will ultimately improve patient, family and staff satisfaction.

Methods: A database was created in Microsoft Access to retrospectively analyze data from all patients undergoing a cardiac catheterization procedure beginning January 1, 2008. Variables included 1) procedure date, 2) procedure room, 3) ASA, 4) sedation versus anesthesia, 5) weight, 6) height, 7) age, 8) diagnosis, 9) intervention versus diagnostic case, 10) scheduled amount of time for case, 11) actual length of case, 12) patient arrival time in procedure room, 13) time patient prep complete, 14) time procedure began, 15) time all access achieved, 16) procedure end time, 17) time patient transferred out of procedure room. To keep diagnoses and interventions consistent, they were each assigned codes to better identify them statistically.

Queries were run from the documented data to find correlations between the variables and information was then transferred into
Microsoft Excel in order to perform the statistical analysis and relevance.

Results: Currently there are 668 subjects with additional enrollment into the database as cases are completed throughout the end of the year. The queries and statistical analysis are in process. Transplant assessments have been analyzed with a sample size of 115 patients. There was a significant difference in the average time to access based on weight; less than or equal to $20 \mathrm{~kg}$ versus greater than $20 \mathrm{kgs}$. There was not a significant difference between anesthesia versus sedation cases; however there did appear to be a significant difference in both the variance and average of case time between anesthesia patients less than $20 \mathrm{kgs}$ and greater than $20 \mathrm{kgs}$; even though this was a small sample.

Conclusion: Reviewing results of the analysis will promote changes in the scheduling process for the pediatric cardiac catheterization lab. Objective scheduling will improve efficiency of the lab and therefore patients, their families, and staff satisfaction as well as improve customer service. Furthermore, results from the data collected will make it possible to develop an objective scheduling tool to assist enhanced schedule forecasting. The implementation of the scheduling tool will be in a staging process after one year of data has been obtained. The increased objectivity and accuracy of scheduling for pediatric cardiac catheterization patients will greatly improve efficiency as well as decrease if not eliminate delays in cases and cases running late.

\author{
Complexity of Cardiac Surgical Procedure Fails to Predict \\ Cardiac ICU Resource Utilization: The Time is Right to \\ Develop an ICU Severity Scoring System \\ Christine Agee MSN, CPNP, Jon Meliones, Andy Lodge, Scott Lawson, \\ Joe Cesari, Michael Camitta, James Jaggers \\ Duke University
}

Background: Predictors of ICU course in postoperative (postop) pediatric cardiac patients remains elusive. The Aristotle Comprehensive Complexity Score (ACCS) was derived by an international expert panel as a means to quantify the complexity for pediatric patients undergoing cardiac surgery. While this score correlates with operative mortality, it may not contain elements which determine patients' ICU resource utilization including length of stay (LOS), variable direct cost, or total cost per case. We hypothesized that for postop pediatric cardiac patients, the ACCS would not predict LOS, variable direct cost, or total cost per case. Methods: We prospectively collected data on patients from 2007 to 2008. During this time protocols were in place to guide postop and ventilatory management. Patient specific clinical data from the CardioAccess database was linked with the corresponding financial data for each patient from our hospital cost accounting system. The data was then imported into a statistical software program for analysis.

Results: 211 patients had complete clinical and financial data. There was a poor correlation between the ACCS and ICU resource utilization including: postop LOS ( $\mathrm{R} 2=0.28)$, variable direct cost $(\mathrm{R} 2=0.20)$, and total cost per case $(\mathrm{R} 2=0.21)$. ACCS showed a weak correlation with diagnosis $(\mathrm{R} 2=0.56)$. There was, however, a strong correlation between postop LOS and ICU metrics including; total ICU LOS ( $2=0.96)$ and ventilation cycle time $(\mathrm{R} 2=0.76)$.

Conclusion: The ACCS does not accurately predict ICU resource utilization of cardiac surgical patients. An ICU severity scoring system for postop pediatric cardiac pts is being developed. This would allow clinicians to predict ICU resource utilization, as well as objectively compare different treatment options in order to 
impact outcomes. A scoring tool would assist in achieving high quality outcomes and potentially identify opportunities to reduce hospital cost and increase hospital revenue.

The Disturbing Dichotomy of Female Gender in Grown-Up Congenital Heart Disease: Higher In-Hospital Mortality and Lower Resource Utilization

Tara Karamlou MD, MSc, Brian S. Diggs PhD, Ross M. Ungerleider $M D$, Karl F. Welke MD

University of Michigan and Oregon Health and Science University

Background: Relative to men, women who undergo cardiac surgery for acquired cardiac disease experience higher morbidity, higher mortality, and increased hospital length of stay (LOS). The association of female gender with mortality and resource utilization following surgery for grown-up congenital heart disease (GUCH), however, is unknown. We sought to determine riskadjusted trends over time for in-hospital mortality and resource utilization in female patients with GUCH and compare these to male patients with GUCH.

Methods: Index cardiac procedures in patients with 12 congenital heart disease diagnostic groups were retrospectively identified using the Nationwide Inpatient Sample 1988-2003. Riskadjusted logistic regression and linear regression models for inhospital mortality, hospital length of stay (LOS), and total hospital charges were constructed using quintiles of years.

Results: $\mathrm{N}=7937$ cases were identified, yielding a national estimate of $40369 \pm 1343$ cases. $N=22663 \pm 739$ (56\%) were females and $17669 \pm 681(44 \%)$ were males. Females outnumbered $(\mathrm{M})$ across all year quintiles, $\mathrm{P}<0.001$. Risk-adjusted mortality declined over time (percent decrease $0.44 \pm 0.22$ per more recent year-quintile; $\mathrm{P}<0.03)$. Estimated risk-unadjusted in-hospital mortality for female patients $(1055 \pm 82 ; 2.6 \%)$ was higher than for male patients $(844 \pm 67 ; 2.1 \%)$. After adjustment for other factors, female gender remained a significant risk-factor for in-hospital death throughout all year-quintiles, despite a decline in overall in-hospital mortality over time (adjusted odds ratio of 1.3). Male gender, however, was significantly associated with longer hospital LOS (parameter estimate $0.80 \pm 24$ days; $\mathrm{P}<0.001)$ and higher total hospital charges $(71 \pm 13$ (X1000 dollars); P < 0.001).

Conclusion: Despite an overall improvement in national outcomes for GUCH over time, female gender continues to be a significant risk-factor for in-hospital death. It is concerning that resource utilization appears to be higher among male patients despite the higher mortality among females, especially in the absence of obvious gender-specific differences in age, comorbidities, or diagnostic group. Further studies examining the underlying reasons for these discrepancies are warranted.

Slow and Steady Wins the Race: A Standardized Feeding Protocol for Infants With Single Ventricle Physiology Decreases the Incidence of Necrotizing Enterocolitis Coleen Miller RN, MS, CPNP, Bronwyn Bartle CPNP, Kelly Nicklas CPNP, Stephanie Bercherer RN, Susan Fussell RD, D. Scott Lawson $C C P$, Sarah Pasquali MD, Jon Meliones MD, Andrew Lodge MD, James Jaggers MD, Piers Barker MD

Duke University Medical Center

Background: While the mortality rate for infants with single ventricle physiology undergoing stage 1 palliation has significantly decreased due to improved surgical and critical care techniques, considerable morbidity persists. In particular, these infants are prone to gastrointestinal complications, with the most serious being necrotizing enterocolitis (NEC). The etiology of NEC in this population is multifactorial and includes diastolic retrograde mesenteric blood flow, decreased postprandial mesenteric blood flow, baseline cyanosis, and periods of low cardiac output. We therefore formed a multidisciplinary team to develop an evidence-based feeding protocol for this high-risk population. Specifically, the protocol defines standardized methods for advancing feeds to goal volume and transitioning to high caloric bolus feeds. The protocol also incorporates a standardized evaluation and treatment plan for NEC based on radiographic and physical exam findings, and patient hemodynamic status. We now hypothesize that this standardized approach will decrease the incidence of NEC without delaying time to full enteral feeds.

Methods: Following IRB approval, retrospective chart review of 22 infants with single ventricle physiology before (control group) and after (treatment group) implementation of the feeding protocol was conducted. Data recorded included baseline cardiac diagnosis, demographics, days required to advance to full goal volume feeds, and the incidence of NEC. NEC events were defined as clinical or radiographic findings concerning for NEC that resulted in stopping enteral feeds and/or providing additional medical therapy. NEC events were categorized as occurring during the advancement to full goal volume or after reaching full goal volume feeds. The numbers of days of treatment for the NEC event were also compared.

Results: There were 10 infants in the control group $(\mathrm{HLHS}=9$, $\mathrm{PA} / \mathrm{IVS}=1)$ and 12 infants $(\mathrm{HLHS}=10, \mathrm{TA}=1, \mathrm{UAVSD}=1$ ) in the treatment group. There was no significant difference between the two groups in regard to age at surgery, birth weight, type of single ventricle defect, type of systemic to pulmonary shunt or mortality. The incidence of NEC was significantly decreased in the treatment group (group $(\mathrm{p}=0.03)$ ). When NEC concerns were identified, the total number of NPO/treatment days were also significantly decreased $(\mathrm{p}=0.02)$ without increasing mortality $(p=0.08)$ in the treatment group. The number of days to full goal volume feeds (control $=11$ days, treatment $=8.5$ days) indicated a trend towards decreased time to full feeds in the treatment group, but this did not reach statistical significance $(\mathrm{p}=0.86)$.

Conclusion: The use of a standardized feeding protocol for infants with single ventricle physiology decreases the incidence of NEC after stage 1 palliation and shortens the duration of NEC treatment with no delay in time to reach full volume enteral feeds. Ongoing evaluation with a larger group should further define the benefits of a standardized approach to feeding infants with single ventricle physiology.

\section{Intravenous Ibuprofen and Closure of Patent Ductus Arteriosus in Preterm Infants \\ Lia Pedersen MD, MPH, Lars Madsen, Finn Ebbesen Aalborg Hospital, Aarbus University Hospital}

Background: Spontaneous closure of patent ductus arteriosus (PDA) in preterm infants is delayed or does not occur. Its incidence in preterm infants is between 30 and $70 \%$, being higher the lower gestational age. A PDA will lead to left to right shunt, increasing risk of intraventricular hemorrage, necrotising enterocolitis (NEC) and bronchopulmonary dysplasia.

We report our experience with IV ibuprofen (Pedea) treatment in a danish neonatal intensive care unit with a mixed population of preterm infants.

Methods: During the period december 2006 to july 2008, 199 infants were born before full 34 gestational weeks. PDA was 
diagnosed clinically and echocardiographically in 31 infants with an incidence of $16 \%$. The PDA was evaluated as moderate or severe, if the $2 \mathrm{D}$ diameter was $>; 2 \mathrm{~mm}$, left atrium/aorta ratio $>1.3$ and the gradient through the duct $<20 \mathrm{mmHg}$. Eighteen infants had a hemodynamical significant PDA and received ibuprofen at median 11 days of life (range 2 û 20). It was given IV as recommended in doses of $10 \mathrm{mg} / \mathrm{kg}$ followed by $5 \mathrm{mg} / \mathrm{kg}$ after 24 and 48 hours. Ratio girls/boys was 11/7, median birth weight $1092 \mathrm{~g}$ (range $810 \hat{\mathrm{u}}$ 1700) and median gestational age 28.4 weeks (range 25.9 û 33.6). Thrombocyte count, serum INR, creatinin/urea, sodium, potassium, body weight and 24 hour-urine output were controlled before, during and after treatment. Echocardiography was repeated 24 to 72 hours after the last ibuprofen dosis.In all infants with PDA at discharge, clinical and echocardiographical follow-up was undertaken after 6 and 12 months or until the PDA was closed.

Results: After the ibuprofen treatment, PDA was only closed in 2 infants $(11 \%)$, was smaller in $10(56 \%)$, although still moderate in 2 , unchanged in $5(28 \%)$ and larger in one infant $(6 \%)$. Six infants received a second treatment with ibuprofen and after that the PDA was smaller in one and unchanged moderate in 5 patients. Of the 16 infants in whom the ductus was patent after ibuprofen, 3 infants had a large PDA with clinical symptoms and underwent surgical closure (17\%), 3 closed spontaneous before discharge, 3 closed spontaneous after discharge, 1 is referred to transcatheter closure, 3 has a small PDA, one is missing to control and one died suddenly after discharge and the PDA was found closed at the autopsy. One infant with a small PDA died of NEC 17 days after ibuprofen treatment. Three others developed NEC, one before treatment and 2 others 5 and 15 days after ibuprofen treatment. No serious side effects were observed.

Conclusion: Our rate of pharmacological closure of PDA with ibuprofen was surprisingly low. There are several randomized controlled trials in preterm infants, which showed a rate of closure of $60-80 \%$. In all these series a very restricted population of preterm infants were included and the treatment given at $48-72 \mathrm{~h}$ of life. We believe that when ibuprofen is given so early, many infants in whom the PDA would close spontaneously, will be treated unnecessary. On the other hand, in our series the treatment was possibly given too late, as the effect is better in the first week of life. Our surgical closure rate was similar to those found in the literature. The ideal timing for treatment is probably between 3 and 7 days of life, and a routine echocardiography in this period will help in decision-making.

Experience of a Nursing Led Peripherally Inserted Central Catheter (PICC) Team in a Congenital Heart Center Deborab King RN, CRNI, Jon Kaufman MD, Eduardo DaCruz MD The Children's Hospital, University of Colorado

Background: Neonates and infants with congenital heart disease (CHD) present significant challenges throughout the perioperative period. Often this population will require central venous access prior to surgical intervention and repair, as well as for an extended period of time afterwards. These patients are at substantial risk for catheter associated blood stream infection, vessel thrombosis as well as the inherent risks of repeated invasive procedures and sedation. The size and venous anatomy of these infants may also present technical challenges for placement of central venous access. There is long term and broad experience with the use of peripherally inserted central catheters (PICCs) in neonates and infants. PICCs are well tolerated for extended periods of time and avoid disruption and damage to the larger central venous catheters placed in femoral, subclavian and internal jugular veins. At The Children's Hospital Denver the PICC team is exclusively led and staffed by dedicated registered nurses (RNs). We present a 33 month review of this team's performance for patients in the Cardiac Intensive Care Unit (CICU).

Methods: A retrospective chart review of 141 neonates and infants under 12 months of age admitted to the CICU from January 2006 to September 2008 who had undergone PICC insertion during this time period. All insertions were performed bedside by PICC RN's using direct visualization, palpation or ultrasound to access peripheral veins. Catheter placement was confirmed radiographically without the use of fluoroscopy. A database included patient demographics, insertion information, reason for removal and catheter duration.

Results: 107 catheters were placed in 91 patients for a success rate of $75.9 \%$. The most common diagnoses were hypoplastic left heart syndrome and transposition of the great vessels. Cyanotic heart lesions accounted for $75 \%$ if the patients. The median age at time of insertion was 43.9 days and the mean weight at time of insertion was $3.6 \mathrm{~kg}$ (range 2.0 to $7.6 \mathrm{~kg}$ ). Ultrasound was used in $20 \%$ of insertions. Central access, defined by superior vena cava or cavoatrial junction placement, was achieved $75.7 \%$ of the time. Procedural complications included 5 arterial punctures (3.5\%), 2 desaturations in non-ventilated patients requiring mask CPAP (no intubations) $(1.4 \%)$, and one hematoma $(0.7 \%)$. Successful completion of therapy occurred in $69 \%$ of patients. Catheter dwell time ranged from 1-71 days with a mean of 17.4 days and a median of 12 days. The infection rate was 2.3 incidences per 1000 catheter days. Catheter insertion success over this time period has also increased.

Conclusion: We demonstrate a successful model of a PICC team for neonates and infants with CHD led by dedicated nurses. Our center experienced an overall high rate of successful central PICC insertions in a diverse and complex patient population, as well as a very low rate of complications. We have also seen an increase in the overall success rate as our staff gains experience with bedside ultrasound. PICC teams staffed by RN's trained in the use of bedside ultrasonography can provide an efficient and safe means for central PICC insertion in the infant with congenital heart disease.

\section{Rounding in the CVICU: New Efficiencies, Improved Communication, Teaching and Patient Care \\ Kerry Sembera MSN, Gay Matthews, Anthony Mott, Dave Nelson, Joseph Rossano}

Texas Children's Hospital, Houston

Background: Miscommunication, lack of efficiencies, and potentially poorly coordinated care may result from ineffective patient rounds in the CVICU. We designed an observation protocol and survey to evaluate these potential problems in the CVICU at Texas Children's Hospital, Houston.

Methods: The observation protocol and survey was designed by cardiology faculty with support of the nursing clinical specialist to survey CVICU nursing, fellows, attendings, and surgical team members, with 87 total respondents. We evaluated communication amongst team members, attitudes and comfort in regard to providing input, potential conflicting opinions between the medical and surgical team in regard to patient care, and how to improve teaching on rounds. This was undertaken from May-June, 2008. Results: $82 \%$ of those surveyed thought communication on rounds needed improving. $73 \%$ of those surveyed were comfortable expressing management opinions to the cardiologist while only $44 \%$ with the surgery team. Only $54 \%$ felt the amount teaching on rounds was satisfactory. 
Conclusion: Based on observational and survey results, we have implemented a new rounding strategy. The medical (attending and fellow) and surgical (surgeon, fellow and physician assistants) teams round together in the morning and there is a representative from both teams on rounds later in the day. This strategy has strongly improved communication between services, enhanced teaching, and positively effected patient care. Follow-up post implementation survey results will also be reported.

\section{Near Infrared Spectroscopic (NIRS) Monitoring During Cardiopulmonary Exercise Testing: Emerging New Tool for Investigating Cardiovascular Physiology in Pediatric Cardiology \\ Michael Danduran MS, Robit Rao MD, George Hoffman MD, \\ Nancy Ghanayem MD, Peter Frommelt MD \\ Children's Hospital of Wisconsin}

Background: Cardiopulmonary exercise testing (CPET) provides assessment of the physiologic limits of integrative responses which are not adequately assessed through the measurement of global or individual organ system function during resting conditions. Blood flow distribution during exercise depends on regional, autonomic and humoral mechanisms affecting vascular resistance resulting in a change in the flow-metabolism relationships as a progressive to exhaustion ensues. Near-infrared spectroscopy (NIRS) provides a non-invasive, continuous method to monitor regional venous-weighted oxyhemoglobin saturation $\left(\mathrm{rSO}_{2}\right)$ reflecting regional oxygen supply-demand economy. We hypothesize that multi-site NIRS will reveal differential desaturation patterns during CPET in normal and pathologic states, and characterize the systemic oxygen consumption to flow-coupling dynamics observed during increasing levels of exercise.

Methods: Thirty-Two control subjects without heart disease and eleven patients with heart disease undergoing CPET were recruited. The eleven patients with pathology included five children with Fontan palliation, two with dilated cardiomyopathy, and one child each with transposition of the great arteries (Mustard), Tetralogy of Fallot, coarctation and heart transplantation NIRS probes were placed on the forehead (cerebral $\mathrm{rSO}_{2}$ ), and three somatic sites: para vertebral space, vastus lateralis, and deltoid muscle. $\mathrm{rSO}_{2}$ was recorded at six second intervals at rest, exercise, and through a five minute recovery period, with an identical protocol for all subjects.

Results: In control subjects, oxygenation in exercising muscle tended to increase early in aerobic exercise and decrease below baseline at the anaerobic threshold (AT), with hyperemia during recovery. The regional oxygenation trends in non-exercising somatic sites showed progressive desaturation with an accelerated slope during the anaerobic phase, followed by rapid recovery and more variable hyperemia. Differences were identified in pathologic states. Two patients with dilated cardiomyopathy with similar ejection fractions (23 and 30\% respectively) were tested. "Patient A" showed accelerated premature desaturation trends in all tissue beds with severe exercise limitations $\left(\mathrm{VO}_{2} \quad 16 \mathrm{ml} / \mathrm{kg} / \mathrm{min}\right)$. "Patient B" showed paradoxical improvement in the cerebral $\mathrm{rSO}_{2}$ consistent with improved cardiac output during the early part of aerobic exercise followed by near normal desaturation trends of all non-cerebral tissue with progressive exercise $\left(\mathrm{VO}_{2}\right.$ $41 \mathrm{ml} / \mathrm{kg} / \mathrm{min}$ ). Fontan patients have a steep initial slope of desaturation in cerebral circulation, while the post AT slope is lower than for normals with a larger rebound after exercise termination in the cerebral bed. Reduced anaerobic exercise capacity in Fontan patients may be secondary to impaired cerebral autoregulation secondary to low systemic venous compliance.
Conclusion: This data highlights the distinct advantage of multisite NIRS monitoring under physiological control in CPET as it provides real time data of flow to demand coupling dynamics in different vascular beds with differing physiological control mechanisms. Specific representative variations in regional patterns of blood flow during incremental exercise are likely to emerge under different pathophysiologic conditions. Further investigation into its clinical significance is warranted.

\author{
Impact: When Healthcare Leaders and Providers \\ Communicate Poorly \\ Maryanne Kessel RN, MBA, Lee Anne Eddy, Kathy Mussatto, \\ Mary Beth Petersen, Maureen Otto \\ Children's Hospital of Wisconsin
}

Background: Communication among healthcare providers directly affects patient care and safety. In 2005, 69\% of sentinel events reported to The Joint Commission (TJC) had communication as their root cause, and $73 \%$ of those communication-related sentinel events resulted in patient deaths, according to TJC National Sentinel Event Root Cause \& Trend Data. In a study sponsored by American Association of Critical Care Nurses, "Silence Kills," key findings included: $84 \%$ of doctors have seen coworkers taking shortcuts dangerous to patients and $88 \%$ of doctors felt they worked with people who showed poor clinical judgment. However, less than $10 \%$ of healthcare providers were willing to directly confront their colleagues about their concerns. Consider the impact: Nearly three in four medical errors are caused by mistakes in interpersonal communication.

Methods: We evaluated current practice used to review quality of care provided. We had established monthly reviews to evaluate the following: diagnostic errors, surgical misadventures, medication \& therapy mishaps, and deaths. Communication errors were never defined or tracked. First goal was to identify and prioritize communication as a "risk factor" in cardiac patient care. Next to determine communication tools that had to be: Believable (valid \& credible), Useable (defined \& able to be implemented), and Practical (user-friendly \& "intuitive"). Tools utilized to provide a balanced approach for both immediate communication and for changing culture. Immediate communication goal was "right data to right person at right time" via SBARR (Situation, Background, Assessment, Recommendation, Response). Changing culture targeted through Crucial Conversations. SBARR education supplied hospital-wide. Crucial Conversations focused on formal and informal leadership within the Herma Heart Center (HHC). Outside consultant (facilitator) hired. Mastery Course: 16 hours of classroom time. Cost \$250/participant (material); External facilitator cost (16 hours) equals $\$ 19,413$. Initial class of 20 attendees of leaders in the heart center. Consideration paid to including early adopters and skeptics. Significant cost of 2 days of salaries for this team. Second phase included 30 Herma Heart Center staff and faculty interested in acquiring these tools. Final education effort included 20 staff, who attended a 4 hour Crucial Conversation exposure class.

Results: 70 HHC team members engaged in an education process that required a significant time commitment and vulnerable exposure to change in the culture of communication. Anecdotal, qualitative observations similar to social sciences describe behavioral changes. The team has reported an increased success in "safe" communications around challenging patient care interactions. Quantitative results pending.

Conclusion: The cardiac leadership team embraced a new approach to communication in an effort to improve patient care. There 
remains the sense of intimidation that is a continued barrier for consistently practicing safe dialogue. We remain challenged to create and sustain an environment where effective, safe communication is embedded into the fabric of the team's culture We need tools for qualitative and quantitative measurement of improvement and the impact on patient outcomes.

End of the Day: "Knowing is not enough; We must apply. Willing is not enough; We must do." -Goethe

Evaluating Potential Swallowing and Feeding Disorders in Neonates After Cardiac Surgical Interventions Elena Ocampo MD, Constance Cephus, RN, Heather Dickerson, MD, Jack Price, MD, Antonio Mott, MD, J. Lynn Jefferies, MD, Joseph Rossano, MD, David Nelson, MD

Baylor College of Medicine, Texas Children's Hospital

Background: The incidence of vocal cord dysfunction and feeding difficulties is well documented in adults after cardiac surgery. In infants and children, there is evidence to suggest a higher incidence, particularly in association with aortic arch interventions, the intra-operative use of transesophageal echocardiography (TEE), and prolonged intubation. There is a known association between post-cardiac surgical airway and laryngeal abnormalities and uncoordinated suck and swallow mechanisms. Feeding intolerance can lead to prolonged hospital stays with the need for nasogastric (NG) and gastric tube (GT) feedings secondary to inadequate ingestion of calories for weight gain and recovery. Vocal cord dysfunction and swallowing abnormalities increase the risk of aspiration. Recovery from cardiac surgery requires vigilance in identifying and addressing vocal cord (VC) and swallowing abnormalities to ensure adequate caloric intake, shorten hospital stay and avoid morbidity associated with unrecognized aspiration. We evaluated a recently instituted standardized approach to identifying and addressing potential feeding and vocal cord problems in neonates who have undergone cardiac surgery in our institution.

Methods: All neonates who underwent cardiac surgery from May 2007 until May 2008 were recruited for this study. Flexible laryngoscopy (ENT) was performed to evaluate VC function after extubation. Suck and swallow were evaluated by an occupational therapist $(\mathrm{OT})$ prior to initiating oral feeds. Initially all neonates underwent a modified barium swallow study (MBSS) to evaluate for aspiration. During the second half of the study period, an MBSS was performed only for abnormal ENT or OT evaluation or if there were other clinical indications.

Results: 107/125 neonates were included in this study. 82/125 had median sternotomies (MS), with 45/82 undergoing aortic arch interventions (AI). 25/125 had thoracotomies (TC). There was no significant difference in age, weight at operation, or duration of intubation between MS and AI; there was shorter duration of intubation for TC. Only the MS group underwent intraoperative TEE.

17/107 (16\%) had abnormal laryngoscopy: (12) left VC paresis, (4) left VC paralysis, (1) bilateral VC paresis. 16/17 had MS, with 14/16 having AI. Only 1/25 TC had abnormal ENT and OT evaluation and was discharged on thickened feedings.

21/107 (20\%) had abnormal OT exam (gag, cough, stridor) all in MS. 24/92 (26\%) had abnormal MBBS. 3/24 (12\%) TC group had aspiration of thin liquids; discharged on thickened feeds.

105 patients survived to hospital discharge. 11(10\%) GT, $11(10 \%)$ NG, $5(4.8 \%)$ were on thickened oral feeds. The length of stay was significantly longer for patients who required GT and NG vs oral feedings $(\mathrm{p}<0.005)$.
Conclusion: The overall incidence of VC dysfunction and swallowing abnormality was low in our population, but was significantly higher in MS vs TC, particularly with AI as reported in the literature. TEE did not appear to increase the risk of VC and swallow abnormality. Neonates who had VC dysfunction and swallow abnormality were more likely to require a GT, home NG feedings or oral thickened feeds. Because of the low incidence of VC abnormality in neonates after thoracotomy, it is reasonable to follow them clinically with further evaluation only if there are concerns.

\section{Gastrointestinal Morbidity for the Hybrid Approach to Hypoplastic Left Heart Syndrome Wendy Luce MD, R Schwartz, W Beauseau, P Giannone, $B$ Hashiguchi, JP Cheatham, M Galantowicz, CL Cua The Heart Center at The Research Institute at Nationwide Children's Hospital}

Background: Neonates undergoing the Norwood surgery for hypoplastic left heart syndrome (HLHS) have the highest risk for necrotizing enterocolitis (NEC) of all congenital heart disease (CHD) patients. The hybrid procedure is another palliative option for HLHS but NEC in neonates undergoing this procedure has not been reported. The goal of this study was to assess the incidence of NEC in patients undergoing the hybrid procedure.

Methods: Retrospective chart review of neonates who underwent the hybrid procedure for the palliation of complex CHD from 4/02 through 4/08 was conducted to determine the incidence of NEC defined as Bell's Stage II+. Demographic, perinatal, perioperative, clinical and procedural data were collected to determine risk factors. Patients were excluded if they underwent the initial procedure $>30$ days of life or if they were transferred back to their home hospital $<10$ days after the procedure. T-tests and Fisher's exact tests were used to compare groups. Odds ratios from bivariate logistic regressions were used to assess odds of being diagnosed with NEC or of death by the explanatory variables. $\mathrm{P}<0.05$ was significant.

Results: Seventy-three patients were reviewed. Sixty-two patients (84.5\%) had a diagnosis of HLHS, with the remainder having other forms of single ventricle physiology. $11.0 \%$ (8/73) developed moderate/severe NEC. Of the patients with NEC, 37.5\% (3/8) died and one patient required surgical intervention. Earlier gestational age $(<37$ weeks) and lower birth weight were associated with NEC. Prenatal diagnosis and APGAR scores did not correlate with NEC. Preoperative variables (mechanical ventilation, sub-ambient oxygen, prostaglandin infusion rate, inotrope use, umbilical venous and arterial lines, enteral feeding, lowest blood $\mathrm{pH}$, highest blood lactate, creatinine $>2.5 \mathrm{mg} / \mathrm{dL}$, AST or ALT $>250 \mathrm{U} / \mathrm{L}$ ) and procedural variations such as timing of septostomy (with or without bands/stent) and mechanism of ductal stent placement (catheter vs. main pulmonary artery incision) were also not associated with the development of NEC. Postoperative inotrope use, time to initiate enteral feedings and time to full enteral feedings did not increase the risk of NEC. Neonates who experienced abdominal distension (odds ratio 14) and bloody stool (odds ratio 6.96) were more likely to have NEC. Length of hospital stay was not increased in patients who developed NEC.

Conclusion: Earlier gestational age and birth weight are risk factors for developing NEC in patients undergoing the hybrid procedure. Clinicians should have a heightened index of suspicion for NEC if bloody stool or abdominal distension is present. Multidisciplinary approaches to better understand abdominal complications in 
neonates undergoing palliation for severe CHD are needed to prevent this devastating morbidity.

ECMO Simulation for New Unit Development Christine Smith BSN, RNC, Anne Ades, MD, Natalie Rintoul, MD, Roberta Hales, RRT-NPS, RN, Patricia Clifford, MSN-RNC, Lauren Gresh, MSN-RNC, Holly Hedrick, MD

The Children's Hospital of Philadelphia

Background: The Special Delivery Unit (SDU) is the first unit in the world designed specifically for mothers carrying fetuses with cardiac anomalies and congenital defects. With state of the art prenatal counselling and diagnostic imaging, infants at high risk for hemodynamic instability and respiratory compromise after birth can be identified. Despite extensive prenatal assessments, some infants have cardiovascular collapse and respiratory decompensation with cord clamping that may not respond to conventional methods of neonatal resuscitation. Since its inception in June 2008, the SDU has not yet required Extracorporeal Membrane Oxygenation (ECMO) but it has been identified as a potential new site for ECMO cannulation.

Methods: An ECMO simulation scenario for the birth of a baby with Congenital Cystic Adenomatoid Malformation (CCAM) status post an Ex Utero Intrapartum (EXIT) procedure requiring Extracorporeal Membrane Oxygenation (ECMO) support was designed. The scenario ran five times with different multidisciplinary teams participating in SDU Simulation training. The ECMO team was required to respond to the simulation with a circuit and cannulation supplies and simulate the initial steps of an ECMO cannulation.

Results: Multiple critical issues were identified. Some of the issues included new paperwork for releasing blood products emergently to the new unit, ECMO team access to restricted areas in the unit, ECMO medications and additives not available in new unit pyxis, identification and isolation of ECMO electrical outlets on head wall. An unexpected concern not encountered previously with our out born population was Vitamin $\mathrm{K}$ administration essential prior to cannulation and anticoagulation with the ECMO circuit. These defects in the process were unexpected based on the rigorous planning that had occurred prior to the simulation.

Conclusion: ECMO cannulation on a patient has not yet occurred in the SDU. The use of simulation training did alert the team to many potential complications that might have occurred if cannulation was needed. Since the simulation training the issues have been resolved leading to smoother process for real-time cannulation. A repeat multi-disciplinary simulation is being planned to ensure that ECMO cannulation in the SDU will be safe and efficient.

The Cycle of Quality Improvement - Delivering the Highest Standards for Qualification of Network Facilities

Jean Cherry RN

Clinical Sciences Institute, Optum Health

Background: Insurance companies face three major challenges providing relevant and sound standards for qualification of network facilities: 1) administering the qualification process involves time and resources; 2) qualification can be time intensive and cumbersome for the facilities that participate; and 3) the network needs to deliver superior outcomes. To address these challenges, we have developed a cycle of Quality Improvement to evaluate programs on an annual basis. This process has proven very effective for qualifying network facilities for both Transplant and Congenital Heart Disease. Here we focus on our experience qualifying CHD facilities that result in documented improved outcomes for our members as a result.

Methods: The Cycle of Quality Improvement starts with a thorough review of the Qualification Criteria for CHD. We created a document listing all criteria with supporting information from the following sources:

- Published evidence,

- Regulatory requirements,

- Professional organizations,

- Government requirements,

- Expert consensus.

Second, Expert Panels - made up of leaders in the CHD field review all qualification criteria against current published evidence and current clinical practice on an annual basis. The criteria must also be approved by our Policy Committee which includes OptumHealth Complex Medical Conditions senior executives, medical and product leadership.

Third, the evaluation is delivered to facilities in the form of a Request for Information (RFI). The RFI is distributed via the web with pre-populated fields from previous RFIs wherever possible. It tracks user comments for each question to help us continually improve the questions, and includes a customer satisfaction questionnaire for additional input from participating providers. It is aligned with the Pediatric Heart RFI and the UNOS standardized RFIs. In addition to qualification, the RFI is also used to provide information for our web portal, and maintain an information database for our case managers.

Results: Our first baseline Satisfaction Survey for the 2008 annual survey process had a $100 \%$ response rate (17 facilities), and $88 \%$ of those surveyed agreed that they were satisfied with the survey process. It is notable that we received similar responses from the 401 Transplant programs that also went through this process, with a $100 \%$ response rate and $74 \%$ agreeing the survey was easy to use.

We have also validated the network's performance. RFI survey data shows that the average CHD discharge mortality rate at qualified facilities is $31 \%$ lower than that at facilities that did not qualify. The average inpatient mortality rate for RACHS 6 patients is $59 \%$ lower at qualified facilities. In terms of efficiency, the average length of stay at a qualified facility is $25 \%$ shorter (10.7 days vs. 14.2 days) than that at an unqualified facility. This holds true for every RACHS category. RACHS 6 patients at qualified facilities average $32 \%$ fewer inpatient days (23 days vs. 34 days).

Conclusion: The Cycle of Quality Improvement is a unique solution that solves key challenges faced by insurance companies, network facilities and even patients. The process reduces resource requirements for survey administration, streamlines the process for participating facilities, and is proven to help build a high performing network of facilities. In addition, the information is used to steer members to facilities where they can expect a reduction in mortality rates and improved outcomes.

Outcome Advantage of Affiliation with a Single Center of Excellence for Pediatric Cardiothoracic Surgical Services Beth Goens MD

University of New Mexico

Background: The division of Pediatric Cardiology at the University of New Mexico (UNM) is a small program with 2000 outpatient 
visits, 2400 echocardiography studies and $<100$ cardiac surgical cases per year. In November of 2003, we established an affiliation with Lucile Packard Children's Hospital (LPCH) to provide outstanding surgical outcomes for our patients. Our affiliation involves 2 weekly teleconferences (one to review diagnostic studies and discuss medical/surgical plans and the other to review social aspects of patient care); collaboration in pediatric rotation in cardiology for UNM residents; and a social work/Child Life project to train parent mentors who provide support for families of new patients needing surgery.

Many insurance providers have been supportive of our affiliation; however, there are some that dictate which center is used for surgical referral. We compared the outcomes of UNM patients cared for within the affiliation to the outcomes of patients cared for at multiple institutions based on insurance company financial arrangements.

Methods: From November 2003 to present, 212 operations were performed at LPCH for patients from UNM. In the same period, 61 operations were performed at 8 other institutions based on insurance provider contracts. We reviewed the mortality and morbidity as assessed by need for re-operation and prolonged hospital stay between the two groups. Re-operations for abnormalities present during the first postoperative hospitalization were included. Staged palliation and late progression of hemodynamic abnormalities requiring re-operation were excluded. The basic Aristotle score was used to grade the complexity of the cases. Occurrence of extra-cardiac congenital abnormalities was noted. Prolonged length of hospital stay (PLOS) was considered $>21$ days.

Results: The two groups varied in complexity. The average Aristotle score for the LPCH patients was 8.3 (median 8, 3.8\% Aristotle $<6,16.5 \%$ Aristotle $>$; 10) and for the other institutions was 6.1 (median 3, 36\% Aristotle $<6,13 \%$ Aristotle $>$; 10). There were 12 patients (5.7\% of all surgeries) in the $\mathrm{LPCH}$ group, without extra-cardiac abnormalities, who had a PLOS $>21$ days. There were 8 patients ( $3.8 \%$ of all surgeries) who had PLOS and had extra-cardiac abnormalities including chromosomal, biliary atresia, prematurity $<30$ weeks. At the other institutions, 5 patients (8.2\% of all surgeries) had PLOS and none of these children had extra-cardiac abnormalities. The incidence of reoperation was $3.3 \%$ at $\mathrm{LPCH}$ and $7.1 \%$ at other institutions. Overall mortality was $2.8 \%$ at $\mathrm{LPCH}$ and $8.2 \%$ at the other institutions.

Conclusion: Our experience includes a small number of patients so that statistical analysis is difficult. However, outcomes based on mortality, PLOS and need for re-operation, were better at LPCH for our UNM patients than at the multiple other institutions despite the more complex patients being sent to LPCH. Much of this can be attributed to the outstanding cardiothoracic team at LPCH. We believe that close communication between the referring cardiologists and the accepting team is essential to the planning of care. This type of communication is established over time with a single institution via teleconferencing and cannot be replicated with multiple institutions.

Morbidity Factors in the Arterial Switch Operation Serban Stoica MD, David Clarke, Debbie Kozik, Steven Goldberg, David Campbell, Mark Twite, Eduardo Da Cruz, Adel Younoszai, Thomas Fagan, Dunbar Ivy, Francois Lacour-Gayet

The Children's Hospital, Denver, CO

Background: In the absence of mortality (discharge and 30 days) in a consecutive series of arterial switch operations (ASO), we sought to identify the morbidity-related outcome measures that best describe the postoperative course of these patients. We therefore analyzed a number of patient and procedure-related factors.

Methods: All patients undergoing ASO between January 2003 through to September 2008 at a single center were enrolled. We examined the following variables: gestational age, age at operation, weight, ventilated going to surgery, enteral feeding preoperatively, basic and comprehensive Aristotle score, primary and associated diagnoses and procedures, redo sternotomy, preoperative left ventricular training, coronary arteries patterns, bypass and crossclamp times, open chest, ECMO and LV function at discharge. The outcomes studied in isolation and combined were length of hospitalization, duration of ventilation and the presence of specific complications: postoperative mechanical support, pacemaker implantation, diaphragmatic plication, neurological deficit persistent at discharge, renal failure requiring dialysis, unplanned early and late reoperations, transplantation and late death. The most representative outcomes were defined by correlation and multivariate analysis was used to identify independent risk factors.

Results: In the 73 consecutive patients enrolled the average basic and comprehensive Aristotle scores were 10.6 and 13.4 respectively, whereas the hospital mortality was zero. Forty four patients had transposition of the great arteries (TGA) with intact septum (60\%), 19 had TGA with VSD (26\%), 8 had TGA/DORV (double outlet right ventricle) with coarctation of the aorta (11\%) and 2 had DORV non-committed VSD (3\%). The coronary patterns were identified as: normal $(66 \%)$, circumflex off right coronary $(17 \%)$, double loop (5\%), single ostium (5\%) and intramural course (7\%). The prevalence of other risk factors was: weight less than $2.5 \mathrm{~kg}$ $11 \%$, no enteral feeding preoperatively $47 \%$, ventilated going to surgery $22 \%, \mathrm{LV}$ retraining $5 \%$, redo sternotomy $10 \%$, sacrifice of an infundibular artery $27 \%$, malaligned commissures $12 \%$, commissure takedown $16 \%$. The median bypass and cross-clamp times were $170 \mathrm{~min}(78-358)$ and $91 \mathrm{~min}(47-180)$ respectively. Fifteen patients $(21 \%)$ had their chest left open and in 6 others $(8 \%)$ the sternum was reopened in intensive care. ECMO was required in 2 patients $(3 \%)$. The median ventilation time and hospital length of stay were 16 (range 4-70) and 3 days (8-28) respectively. One patient died 3 months after the operation and 2 patients were transplanted, 1 with new onset of a viral cardiomyopathy with normal coronary angiogram. The best predictor of morbidity-related outcome was the composite score of: length of hospital stay + ventilation time + weighted complications. By multivariate analysis, independent risk factors for high morbidity were the comprehensive Aristotle score, malalignment of the commissures, and chest reopening.

Conclusion: With the exception of commissural malalignment, traditional risk factors for ASO may be neutralized by careful intraoperative and postoperative management. The comprehensive Aristotle score is a good predictor of postoperative morbidity.

\section{EEG Seizures After Neonatal Cardiac Surgery with High-Flow Bypass and Maximized Oxygen Delivery Strategy \\ Dean Andropoulos M.D., M.H.C.M., DP Nelson M.D., SA Stayer \\ M.D., AR Stark M.D., ED McKenzie M.D., JS Heinle M.D., CD Fraser M.D., Jr., RA Hrachovy, M.D., E Mizrahi M.D. Texas Children's Hospital/Baylor College of Medicine}

Background: EEG seizures are seen in 11-20\% of neonates undergoing complex cardiac surgery with cardiopulmonary bypass with low flow or circulatory arrest.(1,2) The etiology of EEG seizures is thought to be hypoxic-ischemic injury to the brain, and EEG seizures have been correlated with long term neurodevelopmental 
and MRI abnormalities.(1) The objective of this study was to assess neonates for EEG seizures after surgery with high flow CPB and maximized cerebral oxygenation protocols. The hypothesis was that EEG seizures would be minimized.

Methods: Neonates (<30 days) undergoing surgery with hypothermic bypass were enrolled. CPB protocol was $150-\mathrm{ml} / \mathrm{kg} / \mathrm{min}$ flows, $\mathrm{pH}$ stat strategy, hematocrit 30-35\%. Antegrade cerebral perfusion (ACP) was used, DHCA was minimized. ACP flow was guided by transcranial Doppler flow velocity and NIRS. A treatment protocol designed to maintain $\mathrm{rSO}_{2}>50 \%$ was utilized. Anesthetic regimens included fentanyl $100-400 \mathrm{mcg} / \mathrm{kg}$, midazolam $0.3-2 \mathrm{mg} / \mathrm{kg}$, and isoflurane supplementation. Postoperative analgesia/sedation consisted of morphine and/or fentanyl, midazolam and/or lorazepam provided as indicated. No barbiturates or ketamine were used in any patient. 10 lead neonatal EEG placement was used for a 6 hour baseline EEG preoperatively, and 72 hours of continuous postoperative video EEG. Seizure definition: Sudden onset of repetitive, evolving, stereotypical ictal pattern; Defined beginning, middle, and end; Minimum amplitude of 2 \&\#956;V; Minimum duration of 10 seconds.(2) Seizure burden defined as number of seizures multiplied by average duration of each seizure in each patient.

Results: 68 patients were studied: 36 single ventricle, and 32 two ventricle repairs. In 4968 hours of postoperative EEG, no 2V patients had seizures, and $2 \mathrm{SV}$ patients had brief seizures, for an overall incidence of $2.9 \%$. ( $\mathrm{p}=0.49$, SV vs. $2 \mathrm{~V})$. A single $30-$ second episode of seizure activity in a Norwood Stage I palliation patient at hour 72, and a SV patient with a 30 second seizure at hour 7, and 95 second seizure at hour 10. (See Table). Both patients experienced prolonged $\mathrm{rSO}_{2}<45 \%$ (total of 2105 and 668 minutes, respectively), although prolonged low $\mathrm{rSO}_{2}$ was not associated with EEG seizures in the group as a whole $(p=0.1$ Fisher Exact Test).

Conclusion: Previous reports in similar patient populations have noted a median duration of ictal activity of 139 minutes in one study, and mean number of seizures 36 in another, in the 11-20\% of patients with EEG seizures. Our patient cohort exhibits a dramatically reduced EEG seizure burden. Reasons for this reduced seizure burden are likely improved oxygen delivery to the brain in the perioperative period. Our patients also did receive a significant dose of benzodiazepines in the perioperative period.

Table. Seizure Burden.

\begin{tabular}{lll}
\hline Parameter & SV $(\mathrm{n}=36)$ & $2 \mathrm{~V}(\mathrm{n}=32)$ \\
\hline Postop EEG seizures no., (\%) & $2(6)$ & 0 \\
Seizure burden (seizures x seconds) & $4.3 \pm 21.1$ & 0 \\
\hline
\end{tabular}

Data reported as number and percentage of patients for EEG abnormalities, and number of seizures per $\mathrm{pt} \mathrm{x}$ average seizure duration for seizure burden. Changes were statistically nonsignificant by Fisher Exact or $\mathrm{T}$ test.

Bivalirudin anticoagulation in ECLS therapy

Robert Pretzlaff MD, MS, Gary Raff, Richard Yoshikawa, Laura

Kenny, William Dager

University of California, Davis

Background: Bivalirudin is a member of a class of anticoagulants that act as direct thrombin inhibitors (DTI). The archetypical DTI is hirudin. Heparin, as opposed to DTI's, works indirectly to inhibit thrombin. Patients undergoing prolonged extracorporeal circulation require the use of titratable anticoagulation. Heparin has long been the mainstay of such management. Recently heparin anticoagulation has become problematic in some patients due to the occurrence of heparin induced thrombocytopenia (HIT). DTI's offer an alternative to heparin.

Bivalirudin has been studied in patients requiring anticoagulation during percutaneous coronary interventions, and is being evaluated for patients undergoing cardiopulmonary bypass. Recommended bivalirudin dosing for percutaneous coronary procedures is a bolus of $0.75 \mathrm{mg} / \mathrm{kg}$ followed by an infusion of $1.75 \mathrm{mg} / \mathrm{kg} /$ hour. Advantages of bivalirudin are that it is short acting (t1/2 -25 min), non-immunogenic and exhibits reversible binding. This abstract reports our experience using bivalirudin as an anticoagulant in patients undergoing Extracorporeal Life Support (ECLS).

Methods: Chart review of 7 patients receiving bivalirudin as their anticoagulant while undergoing ECLS. ECLS, in all patients, was performed using a closed circuit with a Biomedicus centrifugal pump, Medtronic hollow-fiber oxygenator and a Carmeda-coated circuit. Patients receiving bivalirudin were monitored using partial thromboplastin times (PTT). Target PTT's were determined by the treating team and were measured every two hours. Titration of bivalirudin was made by making $10 \%$ adjustments in the dose until the target PTT's were obtained.

Results: Since 2006, seven patients, age 0 to 444 months and with weights from 2.7 to $68 \mathrm{~kg}$, have received bivalirudin as their anticoagulant for ECLS. In all but one patient, bivalirudin was begun after difficulty with heparin anticoagulation. Indications included heparin-induced thrombocytopenia, as well as a variety of reasons that made heparin management difficult. Two of the patients were infants following surgery for congenital heart disease, and the others were patients with diagnoses including sepsis and congenital diaphragmatic hernia. Five of patients survived their ECLS course and three patients survived to discharge. No patients died as a result of complications of their anticoagulation and no serious complications attributable to bivalirudin were recorded. The average starting dose of bivalirudin was $0.13 \mathrm{mg} / \mathrm{kg} /$ hour (range $0.05-0.22 \mathrm{mg} / \mathrm{kg} / \mathrm{hr}$ ). The highest average dose was $0.47 \mathrm{mg} / \mathrm{kg} /$ hour (range 0.2 û $1.35 \mathrm{mg} / \mathrm{kg} / \mathrm{hr}$ ). The average time receiving bivalirudin was 204 hours (range 49 û 670 hours).

Conclusion: This abstract details the largest series of patients receiving the DTI, bivalirudin, during treatment with ECLS. In our experience, bivalirudin is an effective anticoagulant, and its ease of titration make it an attractive alternative to heparin therapy.

Educating Baccalaureate Nursing Students in a Pediatric Cardiac Step-Down Unit: Case Study of One Clinical Instructor's Experience

Amy Jo Lisanti RN, BSN

The Children's Hospital of Philadelphia

Background: Junior and senior-level baccalaureate nursing students from a large metropolitan university spend a 6-week clinical rotation in a pediatric cardiac step-down unit (PCSDU) of a nearby children's hospital. This experienced cardiovascular nurse began serving as the clinical instructor (CI) for this rotation 18 months ago. This case study provides insight into the educational process for students in a setting with a specialized pediatric patient population with congenital heart disease (CHD).

Initially, many students lacked motivation and verbalized their interest in specialty areas other than the PCSDU. The CI attempted to stimulate interest by teaching CHD and telemetry 
monitoring, but quickly found that students lacked the foundational knowledge to grasp these complex topics. Students experienced difficulty with basic nursing skills such as assessments, documentation, and planning care. The CI needed to enhance students' motivation while focusing on the students' application of the nursing process at an appropriate level.

Methods: The CI utilized the theoretical framework of mastery goal theory (MGT) to enhance students' motivation, interest, and effort in learning. According to MGT, learning tasks presented as short-term goals (STGs) will gain value when linked to a mastery goal of higher importance. Therefore, if specific tasks in clinical education are not directly linked to the mastery goal of becoming a competent practitioner, students may not place value on the clinical experience and may exhibit low motivation.

The CI made short-term goal-setting a priority in the clinical experience. Students were instructed to provide daily, challenging, yet realistic STGs and reflect in a weekly journal how each goal was or was not met. The CI also returned to the "basics" of nursing education and their application in the specialized clinical setting. On-site clinical education included patient assessment, critical thinking, objective documentation, developmental care, and application of the nursing process. Cardiac teaching was focused on normal cardiac anatomy and basic physiological concepts related to cardiac output, rather than CHD. Also, the CI linked specific patient issues with its relevancy to nursing as a whole.

Results: Since implementing these teaching strategies, the CI has observed enhanced motivation in students. They exhibited improvement in utilizing the nursing process and verbalized increased confidence performing basic nursing skills in a developmentally appropriate manner. Students identified alterations in tissue perfusion, gas exchange, and other pertinent nursing diagnoses in patients with CHD. Also, several students expressed interest in pursuing careers in pediatric, cardiovascular nursing. Conclusion: MGT provided a useful framework for this CI to improve the motivation of students in a PCSDU by promoting goal-directed behavior. By focusing clinical education on appropriate STGs and application of the nursing process, students were able to demonstrate the ability to care for complex pediatric patients with CHD. Due to the national nursing shortage, it is imperative that specialty patient care settings, such as the PCSDU, provide positive experiences for students to enhance recruitment of newly graduated nurses. This CI proposes future research to study students' experiences with STGs in the clinical setting, as well as to measure relevant student learning outcomes, using qualitative and quantitative methods, respectively.

Improving Patient Satisfaction Throughout the Hospital Stay for Pediatric Cardiac Catheterization Amanda Green RN, MSN, FNP-C, Bronwyn Bartle MSN CPNP-AC, Edito Mondigo RN BSN, Linda Peterson RN BSN, Rich Campbell RN $B S N$, Kristi Ryan RN BSN

Duke University Medical Center

Background: The Pediatric Cardiac catheterization lab has traditionally had very good patient satisfaction scores; however, the Press-Ganey survey results from the second quarter of this year showed a dramatic decrease in patient satisfaction. The precatheterization, catheterization, and post catheterization experience (which include the post anesthesia care unit, and hospital ward) are complied together under the title "Procedural experience" in the Press - Ganey survey. In order to improve patient satisfaction, we needed to define the areas of dissatisfaction. This was achieved by implementing a patient follow up plan that allowed us to: 1. Make patients aware of the Press - Ganey survey, the importance completing and returning the survey, and how their feedback affects our practice. 2. Define the problem areas during the patient stay. 3. Ultimately improve patient satisfaction. Methods: A team comprised of some of the Pediatric Cardiac Catheterization Lab nursing staff, advanced practice personnel, and pediatric cardiology administration developed several tools. The first was a laminated copy of the Press-Ganey survey posted in the pre-procedure holding room. The second was a $6 \times 9$ index card with a message about the Press-Ganey survey and the value of patient feedback. The third tool was a patient call back worksheet, which was developed to identify the areas of patient care needing improvement. All outpatients who came for a procedure in the pediatric cardiac catheterization lab were either called or e-mailed (determined by patient preference) approximately one week after their procedure. They were asked certain "scripted" open ended questions which gave them the opportunity to reflect on the good and bad experiences surrounding their recent cardiac catheterization lab experience. After the surveys were completed the results were complied, and the results were categorized by positive or negative experience, and further broken down into location of and details of negative experience.

Results: The preliminary results of the patient satisfaction surveys reveled that the greatest percentage of patient dissatisfaction is related to the Post Anesthesia Care Unit experience. Total number of surveys completed as of October 30, 2008, $\mathrm{n}=10$. Of those 10 $\mathrm{n}=5$ were with cardiac catheterizations done with moderate sedation, and $n=5$ were cardiac catheterization with General Anesthesia. There were $n=2$ which had a negative experience, both involved the Post Anesthesia Care Unit.

Conclusion: The pediatric cardiac catheterization experience is multi faceted. And all areas and aspects of care affect the patient experience. The preliminary results of our survey revealed that the negative experiences reflected in the surveys, were most often occurring in the pediatric post anesthesia care unit. Based on the results of this survey we have identified the areas that need the improvement, and have been able to begin to develop an educational and instructional plan for the staff of the Pediatric and Adult post anesthesia care unit regarding our specific patient population and their needs after cardiac catheterization. By educating the staff of the Pediatric and Adult Post anesthesia care units, and educating the patients about the Press-Ganey surveys and their importance, it is our hope that the patient experience will be improved.

Recipe for Success: Acquisition of Oral Feeding Skills Prior to Presentation for Stage 2 Single Ventricle Palliation Bronwyn Bartle RN, MSN, CPNP-AC/PC, Coleen Miller CPNP, Laura Oliver MA CCC-SLP, Maura Baldwin RN, Janel VanSickle $R N$, Piers Barker MD Duke University Medical Center

Background: Infants with single ventricle (SV) physiology frequently fail to achieve full oral feeds at the time of discharge following stage 1 palliation (S1P). The causes for feeding problems are many, and include gastroesophageal reflux (GERD), decreased muscle tone due to a prolonged catabolic state, congestive heart failure and prolonged nasogastric feeds. Additionally, outpatient provider variability can lead to variable success with achieving all oral feeds by presentation for stage II palliation (S2P). The acquisition of oral feeding skills can be viewed as a corollary for overall development and is vital in improving family bonding, maintaining adequate nutrition and preventing the later significant 
morbidity associated with oral aversion. We therefore evaluated whether an intensive program of feeding support as part of a dedicated Single Ventricle Clinic would result in greater success to achieve full oral feeds in this high risk population.

Methods: Following IRB approval, retrospective chart review was performed on all single ventricle patients $(n=43)$ operated on at a single institution between 01/2004 and 10/2008 who returned for S2P. These patients were divided into 2 groups: a control group operated on prior to the creation of the dedicated SV clinic $(n=13)$, and a treatment group operated on after the SV clinic inception $(n=30)$. Patients in the treatment group were further divided into those patients followed at a referring institution $(n=9)$ which did not strictly follow our SV protocol, or those followed in our SV Clinic $(n=21)$. Data collected included demographics, referring institution, birth weight, feeding method, use of anti-reflux medication and weights at discharge from S1P and at presentation for S2P.

Results: Control Group (Pre SV Clinic):

$4 / 13(30 \%)$ achieved all oral feeds prior to S2P; $88 \%$ mean increase from BW to S2P presentation weight; 10/13 (77\%) on anti-reflux medications.

Treatment Group (Post-SV Clinic), followed at our institution.

14/21 (66\%) achieved all oral feeds prior to S2P; Mean \% increase from BW to S2P presentation weight: for all patients, regardless of feeding method $-88 \%$, for patients who achieved all oral feeds û $110 \%$.

Use of anti-reflux medications: for all patients, regardless of feeding method: $17 / 21(81 \%)$ for patients who achieved all oral feeds $11 / 14(79 \%)$.

Treatment Group followed at referring institution.

$1 / 9(11 \%)$ achieved all oral feeds prior to S2P; $88 \%$ mean increase from $\mathrm{BW}$ to $\mathrm{S} 2 \mathrm{P}$ presentation weight.

9/9 $(100 \%)$ on anti-reflux medications.

Conclusion: Aggressive intervention in a dedicated single ventricle clinic with a designated team of $\mathrm{MD}, \mathrm{RN}$, and feeding therapists can help high risk SV infants achieve full oral feeds and increased weight gain compared to standard outpatient care. GERD is a persistent problem in the single ventricle population and is not impacted by feeding method or ability to gain weight. The feeding success of this clinic is built upon the successful introduction of oral feeding skills and the methodical increase of enteral feeding as part of our inpatient SV feeding protocol. Continued monitoring of the success of the dedicated SV clinic will help to identify morbidity trends and allow for targeted interventions for this high risk population.

Technically Difficult: Improving Echoes in Adult Congenital Heart Disease

Desiree Fleck $P h D(c), R N$, Rita Novello

The Children's Hospital of Philadelphia, University of Pennsylvania

Background: Approximately 1 million adults are living with congenital heart disease in the United States today. Of these 1 million adults, approximately half require lifelong follow-up care. Most should be cared for in a regional Adult Congenital Heart Center. Recent guidelines for the management of adult congenital heart care recommend an annual echocardiogram. It remains unclear which diagnostic tests yield the most information for each annual visits and surveillance of prominent issues among adults with congenital heart disease (CHD). Clearly, echocardiography is a mainstay of cardiology care and a widely accepted diagnostic test for adults with CHD. In addition, experience in performing echocardiography adds to the surveillance of adults with congenital heart disease. As echocardiographs are inherently subjective, it makes intuitive sense that a sonographer trained in adult CHD increases the yield of information obtained in theses studies. When setting up a new adult CHD center, having a congenitally trained sonographer may yield increased surveillance techniques allowing for better care in these patients without increasing exposure to radiation or IV dye. Therefore, the purpose of this retrospective chart review was to determine the correlation between echocardiographs and MRI in a cohort of patients in a newly developed ACHD center.

Methods: A retrospective chart review of 400 patients was undertaken. Measurements of ejection fraction, valvular sufficiency or pathology, and right and left ventricular size were obtained. When available, the same measurements were obtained from Computerized tomography or magnetic resonance imaging. Descriptive statistics were used to describe the sample. Pearson $\mathrm{R}$ Correlations were performed.

Results: Within the last 2 years, since hiring a congenitally trained sonographer, 400 echocardiograms were performed. Since the sonographer was hired, the number of echoes has increased allowing for a more robust database and allowing for comparisons from previous echoes. Time to perform echoes decreased over time. These comparisons allow for increased confidence in cardiologists providing care for this burgeoning population.

Conclusion: When setting up an ACHD clinic, adding a congenitally trained sonographer can increase the information available to care for these patients. Echoes can be plagued with difficulties with body habitus, difficult windows making it difficult to get accurate readings. Where appropriate, serial echoes can add information and confidence to the ACHD provider while provider care to patients with CHD. In addition, patients express confidence when having the same person performing their annual echo. Less time is needed to perform the echo. In the presence of "technically difficult" echoes, the sonographer knows the patients and can adjust techniques to obtain the necessary views and measurements for complete and accurate information. Recommendations are made for improving echocardiograms in this difficult population.

\section{Bacteremia is Associated with Aorta-Pulmonary Shunt Thrombosis in Patients with Surgical Single Ventricle Palliation \\ Harun Fakioglu MD, Andrea Sorbello, ARNP Arnold Palmer Hospital for Children}

Background: We have implemented a home surveillance program which has detected patients with aorta-pulmonary shunt thrombosis and has led to prompt intervention and subsequent restoration of adequate pulmonary blood flow without significant complications. This program involves the caregiver monitoring feeding patterns, weight gain, pulse-oximetry and contacting our medical staff if any significant changes are detected in the patient. All the patients who had shunt thrombosis also had bacteremia. Formulating and implementing preventative clinical practice techniques may improve patient outcomes and further decrease the incidence of interstage mortality. Further studies are needed to stratify risks for this patient population. Investigating coagulation disorders and tailoring anticoagulation therapy together with changing post-operative prophylactic antibiotic therapy may decrease shunt thrombosis.

Methods: Data was analyzed retrospectively in patients with single ventricle palliation who presented with signs and symptoms of shunt occlusion. The study was approved by our institutions IRB. 
Hospital medical records, the Cardiac Center databases and outpatient cardiology records were reviewed to identify factors and gather data. We analyzed patients less than 180 days old, underwent surgical palliation for single ventricle with aortapulmonary shunt and who were readmitted for symptoms of shunt occlusion. Three patients were identified between 9/2006 and 8/ 2008 who had documented thrombosis of their aorta-pulmonary shunts on echocardiogram and/or cardiac catheterization.

Results: All patients had their stage I palliation with a modified Blalock-Taussig shunt (BTS) in the first week of life, average age was 5.6 days (range 3-7 days). Two were full term and one was premature; average gestational age at surgery was 38.3 weeks (range 34-41 weeks). The average weight at the time of surgery was 3201gms (range 2804-3700 gms). One patient had delayed sternal closure. Average length of stay for first hospitalization was 40 days (range 14 days to 73 days). Length of intubation postoperatively was 5.6 days (range 3-10 days). The average age at time of readmission was 107 days (range 91-122 days). The average time post-operatively for presentation was 79.7 days (range 39-116 days). All three patients with shunt thrombosis had positive blood cultures and were treated with antibiotics for presumed endovascular infection. One patient had the same bacterial organism as the previous infection in the first hospitalization. Another patient developed bacteremia about sixteen days after a diagnostic cardiac catheterization. All patients were worked up for coagulation disorders. One patient was found to have prothrombin gene 20210 G-A mutation.

Conclusion: In our retrospective study we found three patients who experienced and survived shunt thrombosis with bacteremia. Shunt thrombosis is a significant cause for morbidity and mortality in this group of patients. Infection may be a major factor contributing to a hypercoagulable, low cardiac output state that results in shunt thrombosis. In addition, presence of a heterozygous mutation as a thrombophillic factor may further increase thrombosis. Changing post-op antibiotic prophylaxis may improve bacteremia related shunt thrombosis and thus improve outcomes. This population is too small to make a conclusive argument. However, the information gathered from reviewing these three cases is helpful for constructing a prospective study with larger cohort.

Extubation in the Operating Room after Neonatal and Infant Congenital Heart Surgery

Brian Boe B.S., David S. Cooper, Jeffrey P. Jacobs, Jean Wilhelm, Janet Kreutzer, Paul J. Chai, Harald L. Lindberg, Dien N. Vu, Jeff Miller, Gul H. Dadlani, Albert Saltiel, Arabela Stock, Michelle Smith and James A. Quintessenza

All Children's Hospital, The Congenital Heart Institute of Florida (CHIF), and University of South Florida, St. Petersburg, and Tampa, FL

Background: Potential advantages of early extubation include decreases in ventilator-associated nosocomial infections and use of sedative medications, shorter ICU and hospital stay, and decreased cost. We adopted an aggressive strategy to avoid unnecessary mechanical ventilation that employed extubation in the operating room in selected infants and neonates after open heart surgery. We review our experience.

Methods: A retrospective chart review was performed of all infants $(<365$ days) who underwent open heart surgery from January 2002 to December 2006 at The Congenital Heart Institute of Florida (CHIF). Two subgroups were analyzed:

1) All VSD, AVSD, TOF repair, and Glenn,

2) Neonates $(<31$ days $)$.
Results: In the 5 years reviewed, CHIF performed 3971 cardiothoracic operations, 2293 in infants, 1029 infant open heart operations, and 374 neonatal open heart operations.

$433 / 1029$ infant operations were in subgroup 1: VSD (17\%), AVSD (20\%), TOF (40\%), Glenn (23\%). 263 of the 433 patients $(61 \%)$ were extubated in the operating room with only $4(1.5 \%)$ requiring re-intubation. For these subgroup 1 infants, operative mortality was $1 \%$ if extubated in the operating theater and $6 \%$ if not.

In subgroup 2, 13/274 (3.5\%) of neonates undergoing open heart surgery were extubated in the operating room with one requiring reintubation: TGA/IVS (3), TGA/VSD (2), BT shunt (2), Aoarch hypoplasia/VSD (2), IAA/VSD (1), TOF (1), Truncus (1), AVSD (1). Conclusion: Extubation in the OR after open heart surgery in infants and neonates can be done safely with appropriate patient selection. Further study will help elucidate criteria for application of this strategy.

Incidence of Renal Anomalies Detected by Pre-operative Renal Ultrasound in Infants with Congenital Heart Disease Emilio Quezada MD, J Pilchard, M Ayers, B Agha, B Boe, N Visyak, $S$ Dodson, Jeffrey P. Jacobs, Gul H. Dadlani, David S. Cooper All Children's Hospital, The Congenital Heart Institute of Florida (CHIF), and University of South Florida, St. Petersburg, and Tampa, FL

Background: Infants with congenital heart disease can have other associated congenital anomalies. The incidence of associated renal anomalies in children with congenital heart disease is unknown. Renal insufficiency can cause significant co-morbidity in patients with congenital heart disease. At our institution, over 400 congenital heart surgeries are performed annually and infants with congenital heart disease routinely receive a pre-operative renal ultrasound. We sought to determine the incidence of renal anomalies in this large cohort of patients with congenital heart disease.

Methods: A retrospective chart review was performed in all infants $<60$ days of age who underwent open heart surgery for congenital heart disease at our institution over the four year interval inclusive of 2004 to 2008. The type of congenital heart disease, associated chromosomal anomalies or genetic syndromes, renal ultrasound and any additional renal testing were recorded from the CardioAccess database and the electronic medical record in an Excel database. Results are displayed as a percentage of total patients.

Results: A total of 244 patients met criteria for analysis. The various types of congenital heart disease were: ventricular septal defect (VSD) (1\%), AV canal (5\%), tetralogy of Fallot (TOF) variants $(16 \%)$, truncus arteriosus $(6 \%)$, transposition of the great arteries $(15 \%)$, total anomalous pulmonary venous return $(5 \%)$, biventricular repair of left ventricular outflow tract obstruction (LVOTO) $(25 \%)$, single ventricle (24\%), and other (2\%). The genetics data demonstrated $32 / 244(13 \%)$ patients with abnormal chromosomes, 40/244 (16\%) with associated syndromes (Heterotaxy, Marfan, Down, Trisomy 18, DiGeorge, Turner, or other). Renal ultrasound data was available for 212/244 (87\%) patients. The renal ultrasounds were classified as normal 128/212 (61\%), mild abnormality (no further testing or antibiotic prophylaxis needed) 58/212 (27\%), and major abnormality (further testing or antibiotic prophylaxis was required) $26 / 212(12 \%)$. Of the 26 patients with major abnormalities, 17/26 (65\%) had VCUG stidies with vesiculo-urethral reflux noted in 5/17 (30\%). In addition, 5/26 (19\%) had abnormal chromosomes and 8/26 (31\%) had an identifiable genetic syndrome. The incidence of major renal abnormalities and various congenital heart defects was: TOF variants 6/26 (23\%), biventricular repair of LVOTO 6/26 (23\%), single ventricles $5 / 26(19 \%)$, transposition of the great arteries 
4/26 (15\%), AV canal defects $3 / 26(12 \%)$, other defects $2 / 26(8 \%)$, and $0 / 26(0 \%)$ for VSD, truncus arteriosus and total anomalous pulmonary venous return.

Conclusion: The incidence of significant (major) renal anomalies in infants undergoing heart surgery for congenital heart disease was only $12 \%$. Patients with major renal anomalies had a higher incidence of chromosomal abnormalities and genetic syndromes. Major renal abnormalities appeared to occur more commonly in patients with TOF, LVOTO, single ventricle, transposition of the great arteries and AV canal defects. The use of pre-operative renal ultrasounds to detect associated renal abnormalities may be useful in selected groups of infants undergoing heart surgery for congenital heart disease. Further analysis with a larger cohort will be needed.

Incidence of Intracranial Anomalies as Detected by Pre-operative Brain Ultrasound in Infants with Congenital Heart Disease

Mark Ayers MD, J Pilchard, E Quesado, B Agha, B Boe, N Visyak, $S$ Dodson, Jeffrey P. Jacobs, David S. Cooper, Gul H. Dadlani All Children's Hospital, The Congenital Heart Institute of Florida (CHIF), University of South Florida, St. Petersburg, and Tampa, FL

Background: Infants with congenital heart disease can have other associated congenital anomalies. Central nervous system anomalies, developmental delay and learning disabilities may occur in children with congenital heart disease. Pre- and post-operative CNS injury can cause significant co-morbidity in patients with congenital heart disease. At our institution, over 400 congenital heart surgeries are performed annually and infants with congenital heart disease routinely receive a pre-operative brain ultrasound prior to heart surgery. We sought to determine the incidence of central nervous system anomalies in this cohort of patients with various types of congenital heart disease.

Methods: A retrospective chart review was performed in all infants $<60$ days of age who underwent open heart surgery for congenital heart disease at our institution over the four year interval inclusive of 2004 to 2008. The type of congenital heart disease, associated chromosomal anomalies or genetic syndromes, brain ultrasound and any additional CNS testing were recorded from the CardioAccess database and the electronic medical record in an Excel database. Results are displayed as a percentage of total patients.

Results: A total of 244 patients met criteria for analysis. The various types of congenital heart disease were: ventricular septal defects (VSD)(1\%), AV canal (5\%), tetralogy of Fallot (TOF) variants $(16 \%)$, truncus arteriosus $(6 \%)$, transposition of the great arteries $(15 \%)$, total anomalous pulmonary venous return $(5 \%)$, biventricular repair of left ventricular outflow tract obstruction (LVOTO) $(25 \%)$, single ventricle $(24 \%)$, and other $(2 \%)$. The genetics data demonstrated $32 / 244(13 \%)$ patients with abnormal chromosomes, 40/244 (16\%) with associated syndromes (Heterotaxy, Marfan, Down, Trisomy 18, DiGeorge, Turner, or other). Brain ultrasound data was available for 216/244 (88\%) patients. The brain ultrasounds were classified as normal 174/216 (81\%), mild abnormality (no further testing needed) 23/216 (11\%), and major abnormality (further testing or delay in surgery was required) $19 / 216(8 \%)$. Of the 19 patients with major abnormalities, 2/19 (11\%) had abnormal chromosomes and 2/19 $(11 \%)$ had a known genetic syndrome identified (Turner and Heterotaxy syndromes). The incidence of major intracranial abnormalities and various congenital heart defects was: TOF variants $5 / 19$ (26\%), biventricular repair of LVOTO $5 / 19$ (26\%), single ventricle $4 / 19(21 \%)$, transposition of the great arteries 3/19 (16\%), AV canal defects $1 / 19$ (5\%), VSD $1 / 19(5 \%)$, and $0 / 19$
$(0 \%)$ for truncus arteriosus, total anomalous pulmonary venous return and other.

Conclusion: The incidence of significant (major) intracranial anomalies in infants undergoing heart surgery for congenital heart disease was only $8 \%$. Patients with major central nervous system anomalies had a slightly lower incidence of chromosomal abnormalities and genetic syndromes. Major intracranial abnormalities appeared to occur more commonly in patients with TOF, LVOTO, single ventricle, and transposition of the great arteries. The utility of pre-operative brain ultrasounds to detect associated intracranial abnormalities may not be useful in infants undergoing heart surgery for congenital heart disease. Further analysis with a larger cohort and evaluation of associated post-operative morbidity and mortality is needed.

Bridge-to-Bridge-to-Transplant in the Berlin Heart Era Stephanie Rockett BSN, MSN, Janet C. Bryant RN, W. Robert Morrow $M D$, Elizabeth A Frazier MD, William P. Fiser $M D$, Robert $D B$, Jaquiss $M D$, Michiaki Imamura $M D, P h D$ Arkansas Children's Hospital and University of Arkansas for Medical Sciences

Background: Although there has recently been increasing use of ventricular assist devices (VAD) to bridge children to cardiac transplantation, currently available VAD technology cannot be deployed rapidly. Some patients who are or may become transplant candidates may present in extremis or deteriorate so suddenly that elective implantation of a VAD is not feasible. In such patients, the only alternative is to initiate mechanical support with extracorporeal membrane oxygenation (ECMO), and later transition to VAD support. We sought to evaluate the outcomes for patients supported with bridge-to-bridge technology and compare these outcomes to a contemporary group of patients supported with VAD alone.

Methods: We conducted a retrospective review of all patients supported with intent to transplant with the Berlin Heart VAD system at a single institution between January 2007 and November 2008. Data were abstracted from medical records and an IRB approved cardiac surgical database. Specific variable collected included the underlying cardiac diagnosis, age, size, duration of ECMO support, occurrence of neurologic event, and ultimate outcome. Patients were divided into two groups for the purposes of analysis: Group I $(n=6)$ were patients supported with ECMO and transitioned to VAD and Group II $(n=9)$ were placed on VAD directly. One additional patient was placed on a centrifugal LVAD and transitioned to Berlin Heart, but not analyzed with either group.

Results: The groups were not different in weight (Group I $12.3 \pm 6.9 \mathrm{~kg}$ vs. Group II $-15.8 \pm 12.1 \mathrm{~kg}, \mathrm{p}=0.48$ ) or age (Group I $2.81 \pm 3.36$ yrs. Vs. Group II $3.69 \pm 3.22$ yrs., p = 0.63). Diagnoses did differ between groups: $8 / 9$ patients in Group I had dilated cardiomyopathy and 1/9 had congenital heart disease; 4/6 patients in Group II had acute myocarditis and 2/6 had congenital heart disease. The mean duration of ECMO support in Group I was $6 \pm 4$ days. Overall, 15 of 16 VAD patients remain alive. In Group I, $4 / 6$ have received a transplant and are alive, 1 died after weaning from the device, and 1 remains on support. In Group II, 8/9 received a transplant and are alive and 1 is alive with normal cardiac function after weaning from the device. Of those who have reached an end state (weaned from VAD, transplanted, or died on support), mean support time did not differ between groups (Group I $54 \pm 59$ days vs. Group II $36 \pm 44$ days, $\mathrm{p}=0.53$ ). Two patients sustained strokes in Group I and 4 sustained strokes in Group II. 
Conclusion: Despite the recent introduction of a bridge-totransplant technology designed for use in children, preliminary emergency support with ECMO continues to be necessary for a significant number of patients. Such support is more commonly required in patients with acute myocarditis and rare in children with dilated cardiomyopathy. Outcomes for patients supported with the Berlin Heart VAD are excellent and equivalent whether or not preliminary support with ECMO is required, although a significant incidence of stroke was observed.

Referrals for Fetal Arrhythmias to a Tertiary Care Jess Pilchard MD, B Agha, E Quesado, M Ayers, B Boe, D Nollman, $S$ Atherly, JB Leshko, N Visyak, S Dodson, David S. Cooper, Jeffrey P. Jacobs, James C. Hubta, Gul H. Dadlani

All Childrens Hospital, The Congenital Heart Institute of Florida

$(\mathrm{CHIF})$, and University of South Florida, St. Petersburg, and Tampa, FL

Background: Fetal arrhythmias are a common cause for a perinatal cardiology consultation. Fetal arrhythmias may cause congestive heart failure, hydrops and fetal demise. Fetal echocardiograms can detect congenital heart disease (CHD), fetal arrhythmias, assess for fetal congestive heart failure and provide a cardiovascular profile score. We evaluated the incidence of post-natal arrhythmias in fetuses with pre-natal arrhythmias.

Methods: Retrospective chart review was performed on all patients between 2003 and 2008. The fetal arrhythmias were divided into: Group $1=$ no arrhythmias; Group $2=$ premature atrial contractions(PAC's); Group $3=$ supraventricular tachycardia other than atrial flutter (SVT); and Group $4=$ other arrhythmias. The cardiovascular profile score(CVP) was calculated with scores of 8 or less considered to be significant. Data was analyzed as percentages and p-values were calculated when appropriate using Fisher's Exact test. A P-value less than 0.05 was significant.

Results: A total of 198 patients met inclusion criteria. The incidence of fetal arrhythmias was: Group 1 - 75/198 (38\%); Group 2 - 67/ 198 (34\%); Group 3 - 17/198 (8\%) and Group 4 - 39/198 (20\%). CHD was present in 37/198 (19\%) (Group 1 - 4/75 (5\%); Group 2 - 11/67 (16\%); Group 3 - 5/17 (29\%); Group 4 - 17/39 (44\%)). The CVP score was 8 or less in 26/198 (13\%) patients. The postnatal results were available for 63/198 (32\%). In Group 1 11/12 (92\%) had no arrhythmias. Group 2 had 10/19 (53\%) with no arrhythmias, 2/19 (10\%) with PAC's, $3 / 19$ (16\%) with SVT, and 4/19 (21\%) with other. Group 3 had 4/10 (40\%) with no arrhythmia, 2/10 (20\%) with PAC's, 4/10 (40\%) with SVT. Group 4 had 7/21 (33\%) with no arrhythmias, 1/21 (5\%) with PAC's, 1/21 $(5 \%)$ with SVT and $12 / 21(57 \%)$ with other. The post-natal echocardiograms corresponded to the prenatal fetal echocardiogram (normal-normal or abnormal-abnormal) in 40/54 (74\%).

Overall $72 \%$ of patients normal rhythm or PAC's with congenital heart defects in $<20 \%$ of all patients. Prenatal normal rhythms, PAC's and SVT had a low chance of having CHD 20/159 (12\%) as compared to CHD in patients with other arrhythmias $17 / 39$ (44\%). $\mathrm{P}$-value $=0.001$. The CVP score also suggested more significant fetal congestive heart failure in patients with other arrhythmias 15/39 (38\%) as compared to normal rhythms, PAC's or SVT 11/159 (7\%) $\mathrm{P}$-value $=<0.001$. Normal prenatal rhythm significantly predicted a normal post-natal rhythm 11/12 (92\%) versus 1/12(8\%) P-value 0.025. Prenatal PAC's lead to postnatal SVT or other in $7 / 19(37 \%)$. Patients with prenatal SVT had a $40 \%$ incidence of post-natal SVT or other. Prenatal patients with other arrhythmias had 13/21 62\% incidence of postnatal SVT or other.

Conclusion: Most patients referred for perinatal consult for an arrhythmia will have a normal rhythm or PAC's and a low risk of CHD. The presence of prenatal PAC's or SVT has $>30 \%$ risk of recurrent postnatal arrhythmias in this study.

\section{RoTEM Analysis for Hemostatic Monitoring in Pediatric Cardiac Surgery \\ Hanne Ravn MD, PhD, Jo Boending Andreasen, Anne Mette Hvas \\ Dept of Anesthesia and Intensive Care, Aarhus University}

Background: Successful management of bleeding disorders after congenital heart surgery depends on precise detection of specific coagulation disturbances. Whole blood rotation thromboelastometry (RoTEM, Pentapharm, Germany) presents qualitative continuous clotting profiles with information on initiation (expressed by clotting time, CT, and clot formation time, CFT), propagation (maximum velocity, Max $\mathrm{Vel}$ ) as well as termination of the clot (Maximum clot firmness, MCF). Four standard assays allow testing of: the extrinsic pathway (Ex-TEM), the intrinsic pathway (In-TEM), contribution from fibrinogen (Fib-TEM) and heparinization (Hep-TEM). RoTEM has the advantage of a fast turnaround time, compared to conventional coagulation markers, with results available within $15 \mathrm{~min}$. However, before introducing RoTEM analysis in the management protocol a comparison with conventional coagulation parameters was warranted.

The aim of the study was to investigate whether RoTEM-assays and conventional coagulation assays showed different profiles before and immediately after surgery in pediatric patients undertaken cardiac surgery.

Methods: Sixty children scheduled for primary congenital heart surgery with extracorporeal circulation (ECC) were included in the study. Blood samples were obtained from the arterial cannula immediately prior to heparinization and after weaning off ECC, following administration of protamine to neutralize heparin. Clinicians were unaware of results from RoTEM analysis. Analysis included whole blood RoTEM and conventional coagulation parameters: activated partial thromboplastin time (APTT), prothrombin time (PT relative), fibrinogen, fibrin D-dimer, thrombin time, factor VIII (functional), and platelet count.

Results: Sixty children aged 8 days to 13 years (median 6.4, range 0.4-87.1 months) were enrolled.

Conventional coagulation tests displayed statistically significant changes when pre- and post- ECC values were compared. However, the post-ECC results were within the normal reference range for the majority of the conventional coagulation test results (with the exception of Fibrin D-dimer and platelets) giving only minor support for a targeted intervention. The majority of the RoTEM variables showed abnormal values after ECC, indicating a decreased haemostatic potential. CT increased by $15-25 \%$ in INTEM, EXTEM, FIBTEM and HEPTEM (p-values <0.001), and CFT increased $57-85 \%$, being a significant change in all assay ( $\mathrm{p}$-values $<0.0001$ ) apart from FIBTEM as expected $(\mathrm{p}=0.32)$. The MaxVel showed a significant decrease in all assays ( $\mathrm{p}$-values $<0.005$ ) ranging from $15-39 \%$, and finally, MCF decreased by $10-14 \%$ in both INTEM and EXTEM $(\mathrm{p}<0.0001)$, demonstrating a reduced clot firmness following surgery. These results indicate a higher sensitive by RoTEM analysis compared to conventional coagulation parameters.

Conclusion: Conventional coagulation tests showed minor, though statistically significant, changes after surgery in children. RoTEM analysis proved to be useful in detecting coagulation impairments after congenital heart surgery. RoTEM is already an established tool to monitor the coagulation status in adults following cardiac surgery, but the method should also be considered in the pediatric population where volume overload calls for targeted transfusion therapy to avoid unnecessary volume load. 
A Comprehensive Orientation Program for Newly Recruited Nurses to Cardiac Critical Care: A Collaborative Approach! Cecilia St. George-Hyslop RN, BScN, M Ed, CNCCP (C), Colleen

Gruenwald, David Edgell, Steve Jarvis, Leanne Davidson, Celeste Foreman, Lisa Davey, Karen Dryden-Palmer, Shaf Keshavjee,

Ben Sivarajan, Anne-Marie Guerguerian

Lebatt Family Heart Centre, The Hospital for Sick Children, Toronto, Ontario

Background: The Labatt Family Heart Centre (LFHC) at SickKids, Toronto, provides comprehensive diagnostic, medical and surgical care to children from birth to $18 \mathrm{yrs}$, with congenital and acquired heart disease. Approximately 15-18 children are in the Cardiac Critical Care Unit (CCCU) each day. With ongoing program expansion, the Interprofessional team set a goal to increase the number of cardiac critical care beds to18 from 16. Critical care is a dynamic environment with unpredictable fluctuations in census and acuity levels. Further challenges for CCCU are those that exist in all areas of healthcare today, a scarcity of financial and human resources. The most pressing challenge facing CCCU was the expansion of cardiac critical care, during a significant nursing shortage. These factors combine to create challenges in meeting needs of children and families that require CCU services. This new orientation program required rigorous assessment and evaluation. The new graduate $\mathrm{RN}$, represented a significant portion of available nursing resources for hire. From June to December 2007, 18 nurses were hired, of which 11 were new graduates. In 200810 new graduates and 5 experienced RN's were hired. The priority became provision of an orientation program that would effectively prepare newly recruited nurses for the critical care environment. A comprehensive orientation program was implemented, to facilitate new practitioners in providing competent and safe care to patients and families. The objectives were aligned with the LFHC's goals and strategic directions. The orientation program was enhanced and supported by the Ontario Ministry of Health "New Graduate Nurse Initiative", the funding provided 6 months salary for training.

Methods: The orientation program was revised from a traditional 12 weeks to 9 months of training, which included theory and practice. The theoretical component was a collaborative approach which involved the Interprofessional team in its delivery. It incorporated Simulation Training, Objective Structured Clinical Evaluation's (OSCE's), pre/post testing through Basic Knowledge Assessment Tool in Pediatric Critical Care Nursing (BKAT) examinations. Nurses also began the British Columbia Institute of Technology Critical Care Course, which is an independent study method, via distance learning. This was followed by a preceptorship period of 9 months. This clinical placement is critical to their ongoing development and success in caring for critically ill children and their families. The training of the newly recruited nurses include significant input and contributions of the entire Interprofessional team. The orientation program became rigorous in terms of assessment and evaluation processes. This presentation will include a rubric, to show methods of evaluation.

Results: The success of this orientation program is a direct result of a collaborative approach and an expert Interprofessional team. Short term results appear promising. At SickKids, $1 \mathrm{yr}$ turnover rate is $10 \%$. For Cardiac Critical Care it is $5 \% .1 \mathrm{yr}$ retention rate for the 2007 new graduates is $91 \%$. Other markers of success are based on BKAT results, satisfaction surveys, OSCE's, \& clinical competency checklists.

Conclusion: The success of this orientation program is a direct result of a collaborative approach, a shared vision and an expert Interprofessional team. With this strategy we anticipate being in an over hire situation in the near future. New graduate nurses are an effective recruitment pool for Cardiac Critical Care.

Long Term Follow-up After Surgical Repair of Aortic Coarctation: High Prevalence of Cardiovascular Morbidity

Thais Pedersen MD, Kim Munk, Niels H. Andersen, Kristian

Emmertsen, Erik Lundorf, Vibeke E. Hjortdal

Aarbus University Hospital, Skejby, Aarbus, Denmark

Background: Coarctation of the aorta $(\mathrm{CoA})$ is related to excessive cardiovascular mortality and morbidity despite successful surgical treatment. Late cardiovascular complications include recoarctation, aortic aneurysms, aortic valve dysfunction and systemic hypertension. The goal of this study is to determine the prevalence of late complications 23 to 43 years after operation for CoA.

Methods: 246 patients underwent operative repair of an aortic coarctation between 1965 and 1985 without routine flow-up. In a previously reported retrospective study, we found that 14 died at surgery, 35 died later (median age 49 years) mainly due to cardiovascular disease and 17 were lost to follow-up. Since then, further 7 patients have died, giving a total of 173 current survivors. All survivors were invited to participate in this prospective study which included 24-hour blood pressure measurements, bicycle exercise test, echocardiography and magnetic resonance imaging (MRI). So far, we have examined 82 patients (30 women) with a mean age of 44 years old (range 26-72 years).

Results: Since the primary CoA repair, 20 (24\%) among 82 patients had in the intervening period being referred and treated surgically or by catheter for recoarctation, ascending aortic aneurysms or aortic valve dysfunction. Thirty two $(39 \%)$ were on antihypertensive medication. On 24-hour blood pressure measurements, 21 (26\%) were hypertensive $(>140 / 90 \mathrm{mmHg}$ during daytime or $>135 / 85 \mathrm{mmHg}$ over a 24 hour period), 12 of them despite medication. Thirteen normotensive patients at rest $(22 \%)$ had a hypertensive response on bicycle exercise test (systolic pressure $>220 \mathrm{mmHg}$ ). Exercise performance was reduced in $18(22 \%)$, defined as a maximal achieved workload of less than $85 \%$ of the expected. Echocardiography showed a bicuspid aortic valve in 41 $(50 \%)$, more than trivial aortic or mitral valve insufficiency in 14 $(17 \%)$ and stenosis in 4 patients (5\%). On MRI, we found residual obstruction in the aortic arch in 18 patients (22\%), defined as a minimal luminal diameter in the aortic arch being smaller than $80 \%$ of the aortic diameter at the level of the diaphragm. Ectasia of the ascending aorta, defined as a maximal luminal diameter above $2.0 \mathrm{~cm} / \mathrm{m}^{2}$ was found by MRI in 6 patients $(7 \%)$ and further 5 patients $(5 \%)$ exceeding $2.75 \mathrm{~cm} / \mathrm{m}^{2}$. In consequence of those findings, $12(15 \%)$ have been referred to reintervention due to recoarctation $(\mathrm{N}=8)$ or ascending aortic aneurysm $(\mathrm{N}=4)$.

Conclusion: Repaired coarctation of the aorta is associated with excessive cardiovascular mortality and morbidity. Many patients need reintervention. Long term follow-up must be undertaken.

Coronary Flow Reserve Can Differentiate Stable from Progressive Transplant Coronary Artery Disease in Pediatric Heart Transplant Recipients

Cecile Tissot MD, Bill A. Pietra, Mark M. Boucek, Deborah J. Gilbert, David N. Campbell, D. Dunbar Ivy, Shelley D. Miyamoto

The Children's Hospital, Denver

Background: Transplant coronary artery disease (TCAD) represents the largest cause of late graft loss and the most frequent indication for retransplantation in pediatric recipients. TCAD is often 
asymptomatic and can present with sudden death. Coronary flow reserve (CFR) measures the functional status and microcirculation of epicardial coronary arteries.

Methods: We retrospectively reviewed pediatric heart transplant recipients who had invasive CFR measurements recorded at the time of routine surveillance catheterizations. CFR was obtained from the left anterior descending coronary artery using "a $12 \mathrm{MHz}$ transducer integrated into the tip of a 0.014" guidewire. CFR is defined as the ratio of hyperemic (following intracoronary adenosine) to basal peak Doppler flow velocity (normal $>; 2.5$ ). Patients with and without TCAD were compared. Patients with TCAD were separated into two groups: (1) stable TCAD - no change in angiography or coronary intravascular ultrasound (IVUS) severity after serial evaluation and (2) progressive TCAD - worsening disease or death as a result of TCAD.

Results: 486 measures of CFR were obtained in 176 patients. Fifty (28\%) were diagnosed with TCAD. At the time of diagnosis, CFR was significantly lower but still within the normal range in patients with TCAD compared with no TCAD $(2.7 \pm 0.6$ vs $3.2 \pm 0.6, \mathrm{p}<; 0.01)$. Thirty-six patients $(72 \%)$ had stable and 14 $(28 \%)$ had progressive TCAD. There was no difference between groups in time from transplant to diagnosis of TCAD (5.8 \pm 2.8 , stable vs $6.9 \pm 4$ years, progressive), total ischemic time, gender, age at transplant, weight ratio or number of prior rejections. CFR in the progressive group was lower at the time of diagnosis $(2.2 \pm 0.6$ vs $2.8 \pm 0.8, \mathrm{p}=\mathrm{ns})$ and showed a progressive decline compared to the stable group (1 year post-TCAD diagnosis, $1.6 \pm 0.4$ vs $3.0 \pm 0.8, \mathrm{p}<; 0.01$, cf graph). No patient in the stable group died or required retransplantation as a consequence of TCAD, while all patients in the progressive group died (60\%) or required retransplantation $(40 \%)$. The survival time after diagnosis of TCAD was significantly lower in the progressive group $(1 \pm 1.7$ vs $6.2 \pm 3.8$ years, $\mathrm{p}<; 0.01)$.

Conclusion: CFR remains within the normal range in pediatric recipients with stable TCAD but shows a steady decline over time in patients with progressive TCAD. Increased surveillance and a lower threshold to proceed with retransplantation in patients with an abnormal or decreasing CFR could improve survival in this subset of patients.

Exercise Capacity and Invasive Hemodynamic Measurements Predict Outcome in Children with Cardiomyopathy Cecile Tissot MD, Lisa B Willis, Karrie D Villavicencio,

Adel K Younoszai, Christina M Phelps, Jean L Cavanaugh, Shelley D Miyamoto

The Children's Hospital, Denver

Background: Doppler echocardiography is used to assess diastolic function and correlates with filling pressures in adults. The purposes of this study were to (1) identify echocardiographic measurements that correlate with filling pressures and (2) identify predictive markers for outcome in children with cardiomyopathy.

Methods: All children with dilated (DCM), hypertrophic (HCM) or restrictive (RCM) cardiomyopathy who had a cardiac catheterization and echocardiogram between 2006 and 2008 were reviewed. Patient outcome was divided into: (A) alive and free from transplant or $(\mathrm{T})$ transplanted or dead. Echocardiographic measurements included biplane ejection fraction (EF), DP/Dt of mitral regurgitation (normal $>1200 \mathrm{mmHg} / \mathrm{sec}$ ), mitral inflow and pulmonary vein Doppler, tissue Doppler (E/Ea $<; 8$ normal and $>; 15$ abnormal), color M-mode inflow propagation velocity (vp normal $<45 \mathrm{~cm} / \mathrm{sec}$ ).

Results: Thirty-four catheterizations and echocardiograms were performed in 29 children with an interval between studies of
$5 \pm 58$ days. Diagnosis was DCM in 21, HCM in 4 and RCM in 4 . The mean age was $9.5 \pm 6.2$ years and was lower in the DCM group ( $8 \pm 6.7$ vs $13 \pm 3$ years for RCM and HCM, p $<0.01$ ). There were 14 studies in $\mathrm{A}$ patients and 20 in $\mathrm{T}$ patients. Compared to A patients, the $\mathrm{T}$ group had a lower EF $(33 \pm 21$ vs $49 \pm 13 \%, \mathrm{p}<0.05)$ and mitral inflow $\mathrm{E}$ wave deceleration time (MVE DT, $0.09 \pm 0.03$ vs $0.14 \pm 0.09 \mathrm{sec}, \mathrm{p}<0.05)$. Right atrial pressure, mean PA pressure and wedge pressure (PCWP) measured at catheterization were significantly higher $(\mathrm{p}<0.05)$ in $\mathrm{T}$ patients. Mixed venous saturation, cardiac index and exercise capacity $\left(\mathrm{VO}_{2} \max 15 \pm 4\right.$ vs $\left.26 \pm 7 \mathrm{ml} / \mathrm{min} / \mathrm{kg}, \mathrm{p}<0.05\right)$ were lower in the $\mathrm{T}$ group. MVE DT was lower in patients with a PCWP $>; 12 \mathrm{mmHg}(0.08 \pm 0.03$ vs $0.14 \pm 0.08, \mathrm{p}<0.01)$. E/Ea and vp did not correlate with filling pressures or outcome. DP/Dt $<1200 \mathrm{mmHg} / \mathrm{sec}$ and a higher $\mathrm{E} / \mathrm{Ea}(11.5 \pm 4$ vs $5.5 \pm 1$, $\mathrm{p}<0.05)$ correlated with systolic dysfunction.

Conclusion: In conclusion, catheterization measurements, $\mathrm{VO}_{2} \max$ and MVE DT predicted outcome in children with cardiomyopathy. MVE DT correlated with filling pressures, while DP/Dt and E/Ea correlated with systolic function. Prospective studies in larger populations are needed to confirm these findings and to identify useful non-invasive markers of outcome in children with cardiomyopathy.

Communication and Team Building Skills in the Cardiovascular Intensive Care Unit Mary O'Brien RN, Cynthia Hogan RN, Jeanne Ahern RN, Melvin Almodovar MD Childrens Hospital Boston

Background: In 2005, nationally there were 2500 sentinel events reported to the Joint Commission. The root cause of greater than $70 \%$ of these sentinel events was determined to be a communication failure amongst team members and $70 \%$ of pts involved in these sentinel events died as a result. A group of CICU physicians and nurses at a tertiary care pediatric teaching hospital developed an educational program to reinforce communication strategies in the clinical setting and improve teamwork skills during times of crisis.

Methods: From November 2006 through February 2008, five audits were randomly performed on the day shift each month during organized times of rounds and transfers (nurse to nurse report and admission from the operating room, the cardiac catheterization lab) to assess for the presence of 16 effective communication techniques. In March 2008, five additional audits were performed which concentrated on times of crisis (CPR, semi emergent procedures) and the night shift. In July 2008 an educational program was developed for nurses and physicians to identify effective communication behaviors and how to incorporate these communication behaviors into the clinical setting.

Results: Data was collected prior to training from November 2006 until June 2008 which demonstrated compliance with the 16 behaviors identified. Behaviors were divided into four categories; coordination, awareness, cooperation, and communication. Prior to the initiation of the educational plan the audits demonstrated that CICU team members utilized effective communication strategies in $77 \%$ of opportunities in the category of coordination that includes verbalizing a plan and verbalizing expected timeframes. In the category of awareness, that includes evaluating the environment and verbalizing adjustments in the plan, techniques were observed in $83 \%$ of audited interactions. The category of cooperation, containing requests for external resources, asking for help from the team as needed, verbally requesting team input, 
cross monitoring, verbal assertion, and being receptive to assertion and ideas, occurred in $90 \%$ of exchanges. The final category of communication, containing closed loop, SBAR, thinking aloud, using names, communicating with the patient or parent, and appropriate tone of voice, was evident $88 \%$ of the time. The goal, set as an institutional baseline, is $90 \%$ compliance with all behaviors and a $>10 \%$ improvement in any one element is considered significant improvement. Following this intervention, preliminary data demonstrates improvement in 15 out of the 16 communication techniques and only two behaviors, the use of names and use of the SBAR format, remain below the institutional standard of $90 \%$. The use of names, which is below the institutional benchmark, has shown an $18 \%$ improvement in the compliance with this behavior.

Conclusion: Clarity in communication and teamwork are important skills that a healthcare team must possess to increase reliability and decrease error and harm. Our data suggests that educational programs improve compliance around key communication skills.

Early Diagnosis of Transplant Coronary Artery Disease by Intravascular Ultrasound (IVUS) Improves the Outcome of Pediatric Heart Transplant Recipients Cecile Tissot MD, Bill A. Pietra, Mark M. Boucek, Deborah J. Gilbert, David N. Campbell, Max B. Mitchell, D. Dunbar Ivy, Shelley D. Miyamoto

The Children's Hospital, Denver

Background: Transplant coronary artery disease (TCAD) represents the leading cause of late graft loss after pediatric heart transplantation and the major cause of retransplantation. The diffuse nature of the disease and coronary remodelling renders early diagnosis difficult. The incidence of TCAD is related to the method of surveillance. Intravascular ultrasound (IVUS) allows detection of TCAD at an earlier stage compared to angiography. Coronary flow reserve (CFR) measures the functional status and microcirculation of epicardial coronary arteries.

Methods: We retrospectively reviewed pediatric heart transplant recipients who had IVUS and invasive CFR at the time of routine surveillance catheterization. IVUS was performed in the left anterior descending coronary artery in children whose weight was $>; 25 \mathrm{~kg}$. CFR, defined as the ratio of hyperemic (following intracoronary adenosine) to basal peak Doppler flow velocity, was obtained using "a $12 \mathrm{MHz}$ ultrasound integrated into the tip of a 0.014" guidewire. Abnormal IVUS was defined as intimal thickening $>; 0.3 \mathrm{~mm}$ according to the Stanford classification and abnormal CFR as $<2.5$. Patients with TCAD were separated into 2 groups: (1) angiography diagnosis of TCAD (angiography group) and (2) normal angiography with IVUS diagnosis of TCAD (IVUS group).

Results: 141 IVUS procedures were performed in 57 of 310 transplanted children. Of 36 patients diagnosed with TCAD, 10 (28\%) had normal angiography but abnormal IVUS. There was no difference between the groups in ischemic time, age at transplantation, number of rejections and prior IVUS exams. Weight at transplant was lower in the angiography group $(17 \pm 17$ vs $29 \pm 8.5 \mathrm{~kg}, \mathrm{p}<; 0.01)$. Weight at TCAD diagnosis was higher in the IVUS group $(60 \pm 20$ vs $40 \pm 19 \mathrm{~kg}, \mathrm{p}<; 0.01)$, reflecting the limitation of the procedure in small patients. CFR was higher at the time of TCAD diagnosis in the IVUS group $(3.4 \pm 0.85$, IVUS group vs $2.5 \pm 0.66$, angiography group, $\mathrm{p}=0.01$ ), but by 1 year post-TCAD diagnosis, there was no appreciable difference in CFR between these 2 groups. There was a trend towards shorter time from transplant to diagnosis of TCAD in the IVUS group $(6.9 \pm 3.9$ vs $7.6 \pm 3.5$ years, $\mathrm{p}=\mathrm{ns})$. Eight patients died or required retransplantation as a consequence of TCAD in the angiography group $(8 / 26=30 \%)$ as opposed to one death and no retransplantation in the IVUS group $(1 / 10=10 \%)$. The time from TCAD diagnosis to death was shorter in the angiography group $(1.1 \pm 1.8$ vs $4.3 \pm 3.7$ years, $\mathrm{p}<; 0.01)$.

Conclusion: IVUS was able to detect early TCAD in $18 \%$ of patients with normal angiography in the population suitable for IVUS, the biggest limitation being the size of the patient. Lower CFR at TCAD diagnosis and shorter time to death in the angiography group may reflect TCAD diagnosis at a later stage of the disease. Early TCAD diagnosis by IVUS is associated with improved outcome and decreased need for retransplantation.

The Age-Dependent Effect of Sevoflurane-Induced Neuronal Degeneration in Organotypic Hippocampal Slice Cultures Lisa Wise-Faberowski MD, Estee Piehl MD University of Colorado

Background: Anesthetic induced neurodegenration in the developing brain is a concern especially when infants undergo prolonged exposure to anesthetics for surgical repair for congenital heart disease. Prolonged exposure to volatile anesthetic agents, which act via gamma-amino butyric acid (GABAA) potentiation without $\mathrm{N}$-methyl-d-aspartate (NMDA) antagonsim, such as sevoflurane, has not been investigated. Using a developmental organotypic hippocampal slice model (OHS), we determined the effect of a 5 hour sevoflurane (2\%) exposure on caspase- 3 mediated neuodegeneration. We further determined if the age of rat pup from which the OHS was prepared influenced sevoflurane induced neuronal cell death.

Methods: Organotypic hippocampal slices (OHS) were prepared from rat pups on postnatal days (PND) 4,7 and 14. The slices were exposed to $2.0 \%$ sevoflurane or fresh gas for 5 hours. Hippocampal CA1, CA3, and dentate gyrus neuronal survival, in addition to caspase-3 activity, was assessed immediately (DIV0), 24 hours (DIV1) and 72 hours (DIV3) after exposure to sevoflurane and compared to control slices (fresh gas only).

Results: Neuronal cell death was greatest in OHS prepared from PND 7 rat pups $(\mathrm{p}<0.001)$ in all three regions examined (Figure 1). Caspase-3 activity was noted immediately after exposure to sevoflurane and was significantly different than the control slices (Figure 2).

Conclusion: The etiology for poor neurodevelopmental outcome in infants who have undergone surgical repair for cogenital heart disease is a concern. The implication of anestheitcs as a possible etiology has not been fully evaluated. This in vitro investigation supports an agedependent relationship between proloned sevoflurane $(2.0 \%)$ exposure and caspase- 3 mediated neuronal cell death.

Implementing a Hospital-wide Safety Plan for a High-Risk, Low Volume Therapy

Mary McLellan BSN, RN, CMT, CPN, Katherine Cook

Children's Hospital Boston

Background: High risk procedures/therapies with low patient volume (HRLV) increases mortality and morbidity risk. Prostacycline, a medication to treat pulmonary hypertension, is a chronic continuous intravenous infusion with high-risk for patient fatality should error occur in administration. For the past 5 years, patients receiving prostacycline therapy accounted for $0.7 \%$ of patient days on the inpatient cardiology unit (8E) at Children's Hospital 
Boston. With prostacycline being a HRLV therapy, 8E established guidelines, standard management plans, templated orders and an annual nursing competency for safe prostacycline administration and management. Adverse events related to incorrect prostacycline administration in other hospital locations and outside community care settings.

Methods: The Pulmonary Hypertension Team \& 8E nurses met with staff from the emergency department, procedural areas, intensive care units, pharmacy and anesthesiology to develop a safety plan for this HRLV population. Other institutions and infusion companies administering prostacycline were consulted regarding their safety protocols. These, combined with existing $8 \mathrm{E}$ prostacycline guidelines, were used to create a hospital-wide policy for managing prostacycline patients across the continuum of their hospitalization. A key teaching point list was created communicating the critical aspects of prostacycline administration. This was distributed via interoffice emails, staff/departmental meetings, in-services, and computer based learning module to staff in locations that may encounter patients receiving prostacycline. Prostacycline patients received cards with the list to keep with them at all times should they enter an outside community setting. Coverage $24 / 7$ was established to provide hospital support on prostacycline therapy.

Results: The policy creation \& education provided increased awareness on this HRLV medication. There has been decreased notification time to the Pulmonary Hypertension Team when a prostacycline patient enters the hospital system, decrease time to patient management consultation. There have been decreased adverse safety events at the hospital related to prostacycline therapy.

Conclusion: Patient safety can be improved in the management of low-volume high risk patients through multi-disciplinary, interdisciplinary collaboration on policy creation, implementation and education.

Nuclear Magnetic Resonance Spectroscopy (NMRS): A Means to Evaluate Metabolic Changes within the Developing Cortex and Hippocampus

Lisa Faberowski MD, Natalie Serkova PhD

University of Colorado

Background: Basic science investigation has progressed from genomics, proteomics to metabolomics. NMRS is the present technology for measuring metabolomics. Developing white matter is sensitive to hypoxic-ischemic injury in the very preterm infant and the vulnerability of grey matter, cortex and basal ganglia, increases toward term into the early post-natal brain period. Using a developing rat brain model and NMRS, we evaluated the metabolic differences between hippocampus and cortex.

Methods: Brain tissue from PND 4,7,14 and 21 Sprague Dawley rat pups was immediately harvested, cortices and hippocampi dissected, immediately placed in liquid nitrogen and stored at -80\&\#9702;C. The brain extracts were homogenized and $\mathrm{pH}$ adjusted, separated into lipid and protein components and later analyzed using NMRS.

Results: The developing brain contains a high level of osmoregulator taurine, as well as myo-inositol. The signal of phosphomonoesters (PME, precursors of membrane phospholipids) is particularly high on the 31P-NMR due to the high cell proliferation and membrane synthesis in the developing brain. The hippocampal regions are less metabolically active when compared with cortex; however, the concentration of hippocampal glutamate is increased with brain development.

Conclusion: To evaluate changes in the developing brain, specifically the hippocampus and cortex, in response to hypoxia-ischemia, such as cardiopulmonary bypass, in combination with anesthetic agents it is important to evaluate the individual brain components separately. Brain region specific vulnerability is a function of developmental age. We have a provided a baseline developmental evaluation using NMRS and future investigation will include combinations of chronic hypoxia, hypoxia-ischemia and/or anesthesia.

Nuclear Magnetic Resonance Spectroscopy (NMRS): A Means to Evaluate Metabolic Changes in a Developing Hypoxic Rat Pup Model

Lisa Wise-Faberowski MD, Natalie Serkova PhD

University of Colorado

Background: Chronic hypoxia, as manifested in children with cyanotic congenital heart disease, can result in delayed progression in developmental milestones in growth and learning. Timing of surgical repair and a life of continued cyanosis can have many developmental implications. Using a developmental normoxic and hypoxic rat pup model and NMRS of blood, we attempted to evaluate the developmental metabolic profile of chronic hypoxia.

Methods: Sprague Dawley rat pups of differing postnatal age $(\mathrm{P})$ were maintained in a hypoxic $(<10 \%$ oxygen) or normoxic environment (21\% oxygen) beginning at P2 and continuing until P4 or P7. At this time, upon sacrifice, blood samples were obtained via direct cardiac puncture, immediately frozen in liquid nitrogen and later analyzed via NMRS.

Results: There were no differences in P4 and P7 normoxia, except decreased free fatty acid in $\mathrm{P} 7$, probably due to increased muscle activity.

Hypoxia P4 (vs. normoxia P4) has less metabolic consequences than in older pups; however, an increase in circulating alantoin (renal oxidative damage), creatine (muscle dysfunction), total glutathione (oxidative stress), and alanine (decreased protein synthesis) were seen. No changes in lipid metabolism were observed in hypoxia P4. P7 hypoxia (vs. P7 normoxia) led to dramatic metabolic changes: all previous, hypoxia $\mathrm{P} 4$ changes were enhanced, plus a significant increase in lactate, lysine, $\mathrm{CH}_{2}$ group, glutamine, ornithine and tyrosine. All suggest global hypoxia and acidosis, a significant shutdown of systemic protein synthesis and a decrease in renal amino acid filtration. Also an increase in polyols, (osmotic stress) and changes in blood viscosity were seen. Finally, a dramatic increase in almost all circulating lipids is a direct proof of dramatically decreased lipid uptake and catabolism in the liver, muscle, kidney and adipose tissue. Hydroxybutyrate, a favorite substrate in normal kidney, was also increased in $\mathrm{P} 7$ hypoxia.

Conclusion: NMRS of blood, urine and tissue samples is a reliable means for obtaining the metabolic profile of a living organism at various time points in development. Though this data does not suggest metabolic changes as a function of normal development, it supports a progression of overall metabolic dysfunction with increasing durations of chronic hypoxia. Such analysis can be later used to assess interventions as a function of developmental age and chronic hypoxia.

\section{Reducing Cumulative Trauma: Nursing Interventions to Decrease Cardiac Morbidity \\ Mary Ernst ARNP, MSN, Danielle Emerick, Stefania Sarno, Sue Rojas Miami Children's Hospital}

Background: Factors such as patient volume have been identified as being related to decreased mortality rates. Recently, the notion of focusing solely on mortality rates to compare programs has been 
called into question. Patient volume is associated with numbers of cases performed and does not speak to the complexity of care that is involved. (Welke, Diggs, Karamlou, \& Ungerleider, 2008). Program outcomes should consist of both adjusted mortality rates and morbidity indices. One factor that has not been examined in the literature is the impact of specialty or "dedicated" cardiac intensive care units (CICU) in decreasing cardiac morbidity and mortality. Miami Children's Congenital Heart Institute focuses on risk adjusted mortality rates as well as a philosophy of reducing the cumulative trauma and morbidity for all patients. Some morbidities have been deemed indicative of quality care. Preventive measures for selected indicators were implemented at our institution. These include: (a) pressure ulcers, (b) blood stream infections (BSI), and (c) medication errors.

Methods: In order to identify the environmental attributes of cardiac units, a survey which differentiated the characteristics of CICUs versus shared units (i.e. pediatric intensive care unit [PICU]) was conducted. In addition, the three selected indicators were examined to determine how nursing interventions impacted patient morbidity.

Results: Occurrences of pressure ulcers, rates and length of time between BSIs and rates of near-miss and actual medication errors will be reported. A description of the nursing interventions and how they have or have not reduced morbidity will be presented. Implications for practice and potential research questions surrounding these initiatives will be discussed.

Conclusion: Pressure ulcers, BSIs and medication errors are areas that can be significantly influenced by nursing practice. Nursing interventions focusing on preventive measures, good surgical outcomes and a programmatic approach to reducing cumulative trauma may lead to a decrease in overall morbidity for cardiac patients.

Infant Temperament and Parent Stress in 3 month old infants Post Neonatal Cardiac Surgery for Complex Congenital Heart Disease Deborah Lloyd Torowicz MSN RN PhD (c), Geoffrey Bird, Sharon Irving, Chritra Ravishankar, Danica Sumpter, Gil Wernovsky, Barbara Medoff-Cooper

The Children's Hospital of Philadelphia

Background: Infants who have experienced neonatal surgery for complex congenital heart disease (CHD) have often been described as being both irritable and difficult to soothe. The stress experienced by parents at the time of the infant's CHD diagnosis or hospitalization is well recognized by healthcare professionals and may be further exacerbated by the infant's temperament characteristics. The purposes of the study were to: 1) classify the temperament of critically ill cardiac infants at three months of age, 2) determine if there is a difference in temperament between infants classified as single ventricle (SV) or biventricular (BV), and healthy controls (C), and 3) if a relationship exists between temperament and parent stress.

Methods: One hundred and thirty five infants participated in a prospective NIH funded study on feeding and energy expenditure. All infants with CHD had undergone surgical palliation or correction within the first six weeks of life. Infants were classified by echo prior to discharge as having single ventricle $(n=35)$ or biventricular physiology $(n=37)$ and were compared were healthy controls $(n=63)$.

The Early Infancy Temperament Questionnaire (EITQ) and Parental Stress Index (PSI) (short form) were completed at a 3 month follow-up visit. PSI measures adjustment to the parenting role and has three sub-scores as well as a total stress score (TSS).
The EITQ is a 76 item parent questionnaire for assessing the 9 New York Longitudinal Study temperament characteristics in 1 to 4 month old infants. Data was analyzed using descriptive statistics, correlation, ANOVA, and multivariate regression model.

Results: All EITQ temperament characteristics and PSI sub-scores were significantly correlated for all groups of infants. EITQ ANOVA revealed SV infants were significantly more negative in $\operatorname{mood}(\mathrm{F}=7.14, \mathrm{p}<0.001)$ and less distractible $(\mathrm{F}=5.00$, $\mathrm{p}<0.008)$ than $\mathrm{C}$ infants. In SV infants significant differences were found in adaptability $(p=0.048)$ and demandingness $(\mathrm{p}=0.000)$ compared to control infants on PSI. BV infants were significantly more demanding than control infants $(\mathrm{p}=0.009)$ on the PSI. PSI: Both SV $(p<0.001)$ and BV $(p<0.009)$ were significantly more demanding than control infants. SV infants were significantly less adaptable than control infants ( $\mathrm{p}<0.048$ ). TSS revealed parents of SV infants were significantly more stressed $(\mathrm{p}<0.005)$ and felt significantly less accepting $(\mathrm{p}<0.03)$ and less competent $(\mathrm{p}<0.003)$ in infant care. Regression (SV infants): EITQ mood explained $40 \%$ of the variance $(\mathrm{R} 2=.396, \mathrm{~F}=18.40$, $\mathrm{p}<0.0002)$ for total child domain stress. EITQ distractablility explained $53 \%$ of the variance of the total child domain stress $(\mathrm{R} 2=.528, \mathrm{~F}=31.38, \mathrm{p}<0.0001)$. Life Stress analysis revealed distractibility/soothability accounted for $42 \%$ of the variance $(\mathrm{R} 2=0.416, \mathrm{~F}=19.95, \mathrm{p}<0.0001)$ for Total Life Stress and mood accounted for $36 \%$ of the variance $(\mathrm{R} 2=0.365, \mathrm{~F}=16.14$, $\mathrm{p}=0.0004)$ for Total Life Stress.

Conclusion: Little is known as to why temperament styles in cardiac surgery infants differ from normal infants. Understanding the development styles of infants as well as recognition of parental stress can guide cardiac nurses in developing evidenced based nursing interventions that will maximize parental coping skills and support parents in how to parent complex infants prior to discharge.

\section{Bicuspid Aortic Valve Morphology Does Not Affect Pathologic Phenotype \\ Deepika Thacker MD, Paul M Weinberg MD, Martin St. John Sutton $M D$ \\ The Children's Hospital of Philadelphia}

Background: Bicuspid aortic valve (BAV) occurs in $1-2 \%$ of the general population. Surgery is often required to address ascending aortic (AAO) dilation and/or valvular dysfunction. It is not known if valve morphology determines the clinical pathologic phenotype. Prior studies have shown that patients with fusion of right and non coronary leaflets (R-N) have a more rapid progression of aortic stenosis and aortic regurgitation.

In patients with fusion of the right and left coronary leaflets (R-L), orientation of the valvar orifice might be expected to direct blood flow towards the anterolateral wall of the aorta and thus predispose to the typical anterolateral aortic dilation seen in BAV patients. We hypothesized that patients with R-L fusion would be more likely to have aortic dilation and patients with $\mathrm{R}-\mathrm{N}$ fusion would be more likely to have isolated valve lesions without AAO dilatation.

Methods: Between January 2002 and April 2007, 174 pts with BAV underwent surgery for either aortic root replacement or aortic valve replacement. In 111 patients, intra-operative transesophageal echocardiograms were available and were used to determine valve morphology. Patients were grouped into those with maximum AAO diameter $\geqslant 4.5 \mathrm{~cm}$ and those with maximum AAO diameter $<4.5 \mathrm{~cm}$

Results: Fifty-Six patients ( 45 male; mean age 59 y) had maximum AAO diameter $\geqslant 4.5 \mathrm{~cm}$. Of these, $39(69.7 \%)$ had R-L fusion, 
$12(21.4 \%)$ had R-N fusion and $5(8.9 \%)$ had left-non-coronary fusion (L-N). Fifty-five patients ( 34 male; mean age 57 y) had maximum AAO diameter $<4.5 \mathrm{~cm}$. Of these, $44(80 \%)$ had R-L fusion, $9(16.4 \%)$ had R-N fusion, $2(3.6 \%)$ had L-N fusion. This morphologic distribution is similar to the distribution of R-L, R$\mathrm{N}$ and L-N fusion seen in the general BAV population.

Conclusion: Valve morphology does not affect the aortic dilation which necessitates surgical intervention in patients with BAV. Aortic dilation and valvular dysfunction are likely the result of intrinsic aortic tissue pathology rather than a consequence of altered valvular hydraulics.

The Long Distance Transport of Critically Ill Children on Extracorporeal Life Support

Timothy Thiruchelvam, Mel Membrey, Lara Shekerdemian

The Royal Childrens Hospital, Melbourne, Australia

Background: Since 2003 the Royal Children's Hospital has offered a mobile ECLS service to cannulate and retrieve critically ill paediatric patients who are too unstable for conventional transport. Our team consists of a PICU Specialist, an ECLS nurse, a Perfusionist, and Cardiac Surgeon. Here we describe our experience transporting infants and children sustained on Extracorporeal Life Support (ECLS) to a national paediatric ECLS centre in Melbourne, Australia.

Patients and Methods: Retrospective review of 13 children, mean age 58 months (range 1 day to 16 years), who were transported on ECLS to the Intensive Care Unit at The Royal Children's Hospital Melbourne, between March 2003 and September 2008.

Results: Our team cannuated eleven patients at the referring ICU, three of whom required transthoracic cannulation. Two children were cannulated by their referring centre. Twelve children were placed on ECMO (veno-venous in 3, veno-arterial in 9), and one was placed on Left Ventricular Assist Device. Seven patients were retrieved from interstate PICUs by air (distance $755 \mathrm{~km}$ to $1675 \mathrm{~km}$ ) and six were transported from either a rural or metropolitan ICU by road. The mean duration of retrieval was 12 hours (range 6 to 19 hours). Mean duration of ECLS was 433 hours (range 86 to 1747 hours). Nine patients survived to hospital discharge. There were no significant transport-related complications.

Conclusions: A specialist team can safely perform the cannulation and long-distance transport of critically ill children on ECLS. This does not abrogate the timely referral of potential ECLS candidates and should be reserved for patients who would not otherwise tolerate transport.

Does Mannitol Decrease Cerebral Venous Congestion and Postoperative Irritability in Pediatric Bi-Directional Glenn Patients?

Alan Mannheimer, MD John Lamberti, MD E Sandeep Khanna, MD

Background: The bidirectional cavopulmonary shunt is an operation that diverts systemic venous return from the superior vena cava (SVC) to both lungs. The bidirectional cavopulmonary anastomosis leads to increased venous pressure in the SVC. This can lead to SVC syndrome, pleuropericardial effusions and cerebral venous congestion with increase in cerebral blood volume and increases in ICP*, which may play an important role in post-operative irritability.

After BDG surgery these patients typically do well from a hemodynamic standpoint. However, after extubation, patients frequently demonstrate irritability that requires IV narcotics and sedation. The risk of these medicines is further compromising the patient's respiratory drive and hemodynamics. It is thought *that this irritability is due to post-operative pain and cerebral venous congestion due to an elevated SVC pressure.

Mannitol increases plasma osmolarity, thus drawing fluid from intracellular to extracellular spaces. Thus, it is presumed in our patient population that mannitol can decrease intracranial volume due to its osmolar effect and thus decrease irritability.

Methods: A blinded, randomized prospective clinical trial comparing mannitol versus placebo in managing postoperative irritability as a manifestation of cerebral congestion following bidirectional glenn surgery.

The study period was the immediate 12 hours post-extubation. All subjects were given an initial dose of study fluid $(0.5 \mathrm{gm} / \mathrm{kg}$ mannitol or placebo) following extubation. A study protocol based on the FLACC behavioral scale guided administration of subsequent doses of study fluid or analgesics/sedatives (acetaminophen, morphine, lorazepam). Study fluid was given every 4 hours as needed for FLACC score $>5$. Analgesics and/or sedatives were administered per standardized pain orders for FLACC scores $>3$ between study fluid doses. Diuretics and the oral feeding routine were decided by the medical team and not influenced by study participation.

Results: Thirteen patients were randomized; seven to the mannitol group and six to the placebo group. Irritability was not correlated to length of intubation (Correlation of length of intubation with FLACC $3-4 \mathrm{p}=0.25$ and with FLACC $>5 \mathrm{p}=0.10$ ).

The mean number of minutes to a FLACC $>5$ after a single dose was $64+35$ (placebo) and $101+108$ (mannitol). By ranksum test, these groups are not significantly different $(\mathrm{p}=0.758)$, but those on Mannitol averaged half the amount of time to reach the agitation state, which is suggestive that Mannitol has an effect.

The percent of subjects with a FLACC $>5$ at or before 1, 2, and 3 hours after each infusion was recorded:

\begin{tabular}{lllr}
\hline & 1 hour & 2 hour & 3 hour \\
\hline Control & $62 \%$ & $92 \%$ & $100 \%$ \\
Mannitol & $63 \%$ & $74 \%$ & $78 \%$ \\
\hline
\end{tabular}

Conclusions: While the data may be suggestive that mannitol might have a small beneficial effect in treating some post-operative irritability following bi-directional glenn repair, there was no statistically significant difference between the two groups. However, mannitol's use in this complex patient population may still be an interesting topic for future study.

Panel Reactive Antibody in Pediatric Heart Transplant Candidates Bridged to Transplant with Ventricular Assist Devices

Matthew J O'Connor, Curt Lind, Dimitrios Monos, J William Gaynor, Beth D Kaufman

Divisions of Cardiology, Pathology and Laboratory Medicine, and Cardiothoracic Surgery, The Children's Hospital of Philadelphia and the Division of Cardiology, Children's Hospital Los Angeles

Background: Use of ventricular assist devices (VAD) as bridge to heart transplant $(\mathrm{HT})$ in children is accepted therapy for end-stage heart failure. VAD use in the adult population is associated with the production of antibodies against human leukocyte antigens as measured by panel reactive antibody (PRA); the extent and 
significance of this phenomenon in pediatric HT has not been well described.

Methods: Medical records of all patients (pts) bridged to HT with VAD at our institution were reviewed. Changes in PRA pre and post HT were examined. Pts with class I or II PRA $\geqslant 10 \%$ (PRA+) were compared to pts with PRA $<10 \%$ (PRA-) for outcomes post HT. All values are reported as mean $\pm \mathrm{SD}$ (when applicable). $\mathrm{P}$ values $\leqslant 0.05$ were considered significant.

Results: From 1998-2008, VAD as bridge to HT was used in 19 pts, age $12.9 \pm 6$ years (y). Diagnoses included cardiomyopathy in $16(84 \%)$ and congenital heart disease (CHD) in $3(16 \%)$. Mean VAD duration was 100.6 (range 8-424) days. VAD complications included reoperation for bleeding (37\%), bacteremia (74\%), stroke (16\%), and dialysis (5\%). VAD was successful as bridge to HT in $15 / 19$ (79\%). $10 / 15$ of those transplanted are alive $4.9 \pm 3 \mathrm{y}$ post HT.
Pre and post VAD PRA data were available for 16 pts. 1 pt with CHD was PRA+ pre VAD; 6 pts previously PRA- became PRA+ post VAD (see Table 1). 9 pts were PRA+ post HT, which was sustained in 6 pts at mean $4.0 \pm 3$ y post HT. PRA was donor specific in $4 / 9$ pts. Plasmapheresis was performed in 3 PRA+ pts at time of HT.

No significant differences were detected between PRA+ pts post HT and those PRA - post HT with regard to age, diagnosis, ECMO, pre HT blood product exposure, bacteremia on VAD, or VAD duration. Rejection and survival did not differ between groups.

Conclusion: VAD successfully bridged $79 \%$ of pts to HT. While elevated PRA was observed in $44 \%$ of pts post VAD and in $60 \%$ of VAD pts post HT, sensitization was not uniform, could not be predicted, and did not adversely affect post HT outcome in this series. 